\title{
BTS/ICS guideline for the ventilatory management of acute hypercapnic respiratory failure in adults
}

\author{
A Craig Davidson, ${ }^{1}$ Stephen Banham, ${ }^{1}$ Mark Elliott, ${ }^{2}$ Daniel Kennedy, ${ }^{3}$ Colin Gelder, ${ }^{4}$ \\ Alastair Glossop, ${ }^{5}$ Alistair Colin Church, ${ }^{6}$ Ben Creagh-Brown, ${ }^{7}$ James William Dodd, ${ }^{8,9}$ \\ Tim Felton, ${ }^{10}$ Bernard Foëx ${ }_{1}{ }^{11}$ Leigh Mansfield, ${ }^{12}$ Lynn McDonnell, ${ }_{1}^{13}$ Robert Parker, ${ }^{14}$ \\ Caroline Marie Patterson, ${ }^{15}$ Milind Sovani, ${ }^{16}$ Lynn Thomas, ${ }^{17}$ BTS Standards of \\ Care Committee Member, British Thoracic Society/Intensive Care Society Acute \\ Hypercapnic Respiratory Failure Guideline Development Group, On behalf of the \\ British Thoracic Society Standards of Care Committee
}

- Additional material is published online only. To view please visit the journal online (http://dx.doi.org/10.1136/ thoraxjnl-2015-208209).

For numbered affiliations see end of article.

Correspondence to Dr A C Davidson, BTS, 17 Doughty Street, London WC1N 2PL, UK craigdavidson@doctors.org.uk

Healthcare providers need to use clinical judgement, knowledge and expertise when deciding whether it is appropriate to apply recommendations for the management of patients. The recommendations cited here are a guide and may not be appropriate for use in all situations. The guidance provided does not override the responsibility of healthcare professionals to make decisions appropriate to the circumstances of each patient, in consultation with the patient and/or their guardian or carer.

Received 17 December 2015 Accepted 10 January 2016

\section{SLinked}

- http://dx.doi.org/10.1136/ thoraxjnl-2016-208281

\section{CrossMark}

To cite: Davidson $A C$, Banham S, Elliott $M$, et al. Thorax 2016;71:ii1-ii35.

\section{SUMMARY OF RECOMMENDATIONS}

Principles of mechanical ventilation

Modes of mechanical ventilation

Recommendation

1. Pressure-targeted ventilators are the devices of choice for acute NIV (Grade B).

Good practice points

- Both pressure support (PS) and pressure control modes are effective.

- Only ventilators designed specifically to deliver NIV should be used.

\section{Choice of interface for NIV}

Recommendation

2. A full face mask (FFM) should usually be the first type of interface used (Grade D).

Good practice points

- A range of masks and sizes is required and staff involved in delivering NIV need training in and experience of using them.

- NIV circuits must allow adequate clearance of exhaled air through an exhalation valve or an integral exhalation port on the mask.

Indications for and contra-indications to NIV in AHRF

\section{Recommendation}

3. The presence of adverse features increase the risk of NIV failure and should prompt consideration of placement in high dependency unit (HDU)/intensive care unit (ICU) (Grade C).

Good practice points

- Adverse features should not, on their own, lead to withholding a trial of NIV.

- The presence of relative contra-indications necessitates a higher level of supervision, consideration of placement in HDU/ICU and an early appraisal of whether to continue NIV or to convert to invasive mechanical ventilation (IMV).

Monitoring during NIV

Good practice points

- Oxygen saturation should be continuously monitored.

- Intermittent measurement of $\mathrm{pCO}_{2}$ and $\mathrm{pH}$ is required.
ECG monitoring is advised if the patient has a pulse rate $>120 \mathrm{bpm}$ or if there is dysrhythmia or possible cardiomyopathy.

Supplemental oxygen therapy with NIV

Recommendations

4. Oxygen enrichment should be adjusted to achieve $\mathrm{SaO}_{2}$ 88-92\% in all causes of acute hypercapnic respiratory failure (AHRF) treated by NIV (Grade A).

5. Oxygen should be entrained as close to the patient as possible (Grade C).

Good practice points

- As gas exchange will improve with increased alveolar ventilation, NIV settings should be optimised before increasing the $\mathrm{FiO} 2$.

- The flow rate of supplemental oxygen may need to be increased when ventilatory pressure is increased to maintain the same $\mathrm{SaO} 2$ target.

- Mask leak and delayed triggering may be caused by oxygen flow rates $>4 \mathrm{~L} / \mathrm{min}$, which risks promoting or exacerbating patient-ventilator asynchrony. The requirement for high flow rates should prompt a careful check for patientventilator asynchrony.

- A ventilator with an integral oxygen blender is recommended if oxygen at $4 \mathrm{~L} / \mathrm{min}$ fails to maintain $\mathrm{SaO} 2>88 \%$.

Humidification with NIV

Recommendation

6. Humidification is not routinely required (Grade D).

Good practice point

Heated humidification should be considered if the patient reports mucosal dryness or if respiratory secretions are thick and tenacious.

Bronchodilator therapy with NIV

Good practice points

- Nebulised drugs should normally be administered during breaks from NIV.

- If the patient is dependent on NIV, bronchodilator drugs can be given via a nebuliser inserted into the ventilator tubing. 


\section{Sedation with NIV}

\section{Recommendations}

7. Sedation should only be used with close monitoring (Grade D).

8. Infused sedative/anxiolytic drugs should only be used in an HDU or ICU setting (Grade D).

9. If intubation is not intended should NIV fail, then sedation/ anxiolysis is indicated for symptom control in the distressed or agitated patient (Grade D).

Good practice point

In the agitated/distressed and/or tachypnoeic individual on NIV, intravenous morphine $2.5-5 \mathrm{mg}$ ( \pm benzodiazepine) may provide symptom relief and may improve tolerance of NIV.

\section{NIV complications}

\section{Good practice points}

- Minor complications are common but those of a serious nature are rare. Patients should be frequently assessed to identify potential complications of NIV.

- Care is needed to avoid overtightening of masks.

- Previous episodes of ventilator-associated pneumothorax warrant consideration of admission to HDU/ICU and use of NIV at lower than normal inspiratory pressures.

- The development of a pneumothorax usually requires intercostal drainage and review of whether to continue with NIV.

\section{Sputum retention}

\section{Recommendations}

10. In patients with neuromuscular disease (NMD), mechanical insufflation and exsufflation should be used, in addition to standard physiotherapy techniques, when cough is ineffective and there is sputum retention (Grade B).

11. Mini-tracheostomy may have a role in aiding secretion clearance in cases of weak cough (NMD/chest wall disease (CWD)) or excessive amounts (COPD, cystic fibrosis (CF)) (Grade D).

\section{Modes of IMV}

\section{Recommendations}

12. Spontaneous breathing should be established as soon as possible in all causes of AHRF (Grade C).

13. Controlled IMV may need to be continued in some patients due to severe airflow obstruction, weak muscles leading to poor triggering or to correct chronic hypercapnia (Grade C).

Good practice point

In obstructive diseases, controlled IMV should be continued until airway resistance falls.

\section{Table 1 SIGN grades of recommendations}

A At least one meta-analysis, systematic review, or RCT rated as $1++$, and directly applicable to the target population; or A body of evidence consisting principally of studies rated as $1+$, directly applicable to the target population and demonstrating overall consistency of results

B A body of evidence including studies rated as $2++$, directly applicable to the target population and demonstrating overall consistency of results; or Extrapolated evidence from studies rated as $1++$ or $1+$

C A body of evidence including studies rated as $2+$, directly applicable to the target population and demonstrating overall consistency of results; or Extrapolated evidence from studies rated as $2++$

D Evidence level 3 or 4 ; or Extrapolated evidence from studies rated as $2+$
Invasive ventilation strategy

Recommendations

14. During controlled ventilation, dynamic hyperinflation should be minimised by prolonging expiratory time (I:E ratio 1: 3 or greater) and setting a low frequency (10-15 breaths/min) (Grade C).

15. Permissive hypercapnia (aiming for $\mathrm{pH}$ 7.2-7.25) may be required to avoid high airway pressures when airflow obstruction is severe (Grade D).

16. Carbonic anhydrase inhibitors should not be routinely used in AHRF (Grade C).

Positive end expiratory pressure

Recommendation

17. Applied extrinsic positive end expiratory pressure (ePEEP) should not normally exceed $12 \mathrm{~cm}$ (Grade C).

\section{Sedation in IMV}

\section{Recommendation}

18. Sedation should be titrated to a specific level of alertness (Grade B).

\section{Patient-ventilator asynchrony}

Recommendations

19. Ventilator asynchrony should be considered in all agitated patients (including NIV) (Grade C).

20. As patients recover from AHRF, ventilator requirements change and ventilator settings should be reviewed regularly (Grade C).

\section{Use and timing of a tracheostomy}

\section{Recommendations}

21. Performing routine tracheostomy within 7 days of initiating IMV is not recommended (Grade A).

22. The need for and timing of a tracheostomy should be individualised (Grade D).

Good practice points

- In AHRF due to COPD, and in many patients with NMD or obesity hypoventilation syndrome (OHS), NIV supported extubation should be employed in preference to inserting a tracheostomy.

- In AHRF due to NMD, alongside discussion with the patient and carers, the decision to perform tracheostomy should be multidisciplinary and should involve discussion with a home ventilation unit.

\section{Management of hypercapnic respiratory failure Prevention of AHRF in AECOPD \\ Recommendations}

23. In AHRF due to AECOPD controlled oxygen therapy should be used to achieve target saturations of $88-92 \%$ (Grade A).

Good practice point

Controlled oxygen therapy should be used to achive a target saturation of $88-92 \%$ in ALL causes of AHRF.

\section{Role of NIV in AECOPD}

\section{Recommendations}

24. For most patients with AECOPD, the initial management should be optimal medical therapy and targeting an oxygen saturation of $88-92 \%$ (Grade A).

25. NIV should be started when $\mathrm{pH}<7.35$ and $\mathrm{pCO}_{2}>6.5 \mathrm{kPa}$ persist or develop despite optimal medical therapy (Grade A). 
26. Severe acidosis alone does not preclude a trial of NIV in an appropriate area with ready access to staff who can perform safe endotracheal intubation (Grade B).

27. The use of NIV should not delay escalation to IMV when this is more appropriate (Grade C).

28. The practice of NIV should be regularly audited to maintain standards (Grade C).

\section{Starting NIV in COPD}

Good practice points

- Arterial blood gas (ABG) measurement is needed prior to and following starting NIV.

- Chest radiography is recommended but should not delay initiation of NIV in severe acidosis.

- Reversible causes for respiratory failure should be sought and treated appropriately.

- At the start of treatment, an individualised patient plan (involving the patient wherever possible) should document agreed measures to be taken in the event of NIV failure.

\section{Prognostic features relating to use of NIV in COPD} Recommendations

29. Advanced age alone should not preclude a trial of NIV (Grade A).

30. Worsening physiological parameters, particularly $\mathrm{pH}$ and respiratory rate (RR), indicate the need to change the management strategy. This includes clinical review, change of interface, adjustment of ventilator settings and considering proceeding to endotracheal intubation (Grade A).

Good practice point

If sleep-disordered breathing pre-dates AHRF, or evidence of it complicates an episode, the use of a controlled mode of NIV overnight is recommended.

\section{Duration of NIV in COPD}

\section{Recommendation}

31. NIV can be discontinued when there has been normalisation of $\mathrm{pH}$ and $\mathrm{pCO}_{2}$ and a general improvement in the patient's condition (Grade B).

Good practice points

- Time on NIV should be maximised in the first $24 \mathrm{~h}$ depending on patient tolerance and/or complications.

- NIV use during the day can be tapered in the following 2-3 days, depending on pCO2 self-ventilating, before being discontinued overnight.

\section{Optimising NIV delivery and technical considerations \\ Good practice point}

Before considering NIV to have failed, always check that common technical issues have been addressed and ventilator settings are optimal (table 3).

\section{Indications for IMV in AECOPD}

Recommendations

32. IMV should be considered if there is persistent or deteriorating acidosis despite attempts to optimise delivery of NIV (Grade A).

33. Intubation should be performed in respiratory arrest or periarrest unless there is rapid recovery from manual ventilation/ provision of NIV (Grade D).

34. Intubation is indicated in management of AHRF when it is impossible to fit/use a non-invasive interface, for example, severe facial deformity, fixed upper airway obstruction, facial burns (Grade D).

35. Intubation is indicated where risk/benefit analysis by an experienced clinician favours a better outcome with IMV than with NIV (Grade D).

\section{Outcome following NIV or IMV in AECOPD \\ Recommendations}

36. Prognostic tools may be helpful to inform discussion regarding prognosis and with regard to the appropriateness of IMV but with the caveat that such tools are poorly predictive for individual patient use (Grade B).

37. Clinicians should be aware that they are likely to underestimate survival in AECOPD treated by IMV (Grade B).

38. Clinicians should discuss management of possible future episodes of AHRF with patients, following an epsiode requiring ventilatory support, because there is a high risk of recurrence (Grade B).

\section{Acute asthma}

Recommendations

39. NIV should not be used in patients with acute asthma exacerbations and AHRF (Grade C).

40. Acute (or acute on chronic) episodes of hypercapnia may complicate chronic asthma. This condition closely resembles COPD and should be managed as such (Grade D).

\section{Non-CF bronchiectasis}

\section{Recommendations}

41. In patients with non-CF bronchiectasis and AHRF, controlled oxygen therapy should be used. (Grade D)

42. In patients with non-CF bronchiectasis, NIV should be started in AHRF using the same criteria as in AECOPD (Grade B).

43. In patients with non-CF bronchiectasis, NIV should usually be tried before resorting to IMV in those with less severe physiological disturbance (Grade $\mathrm{C}$ ).

44. In non-CF bronchiectasis, the patient's clinical condition prior to the episode of AHRF, and the reason for the acute deterioration, should be evaluated and used to inform the decision about providing IMV (Grade C).

Good practice points

- In patients with non-CF bronchiectasis, the precipitating cause is important in determining short-term prognosis.

- Health status prior to the episode of AHRF is an important predictor of outcome.

\section{Cystic fibrosis}

\section{Recommendations}

45. In patients with $\mathrm{CF}$, controlled oxygen therapy should be used in AHRF (Grade D).

46. In patients with CF, NIV is the treatment of choice when ventilatory support is needed (Grade C).

47. In patients with CF, specialised physiotherapy is needed to aid sputum clearance (Grade D).

48. In patients with $\mathrm{CF}$, a mini-tracheostomy combined with NIV may offer greater chance of survival than resorting to IMV. (Grade D)

Restrictive lung diseases

\section{NMD and CWD}

Recommendations

49. Controlled oxygen therapy should be used in patients with NMD or CWD and AHRF (Grade D). 
50. NIV should almost always be trialled in the acutely unwell patients with NMD or CWD with hypercapnia. Do not wait for acidosis to develop (Grade D).

51. In patients with NMD or CWD, NIV should be considered in acute illness when vital capacity (VC) is known to be $<1 \mathrm{~L}$ and $\mathrm{RR}>20$, even if normocapnic (Grade D).

52. In patients with NMD or CWD, consider controlled ventilation as triggering may be ineffective (Grade D).

53. In NMD or CWD, unless escalation to IMV is not desired by the patient, or is deemed to be inappropriate, intubation should not be delayed if NIV is failing (Grade D).

Good practice points

- Individuals with NMD and CWD who present with AHRF should not be denied acute NIV.

- NIV is the ventilation mode of choice because patients with NMD or CWD tolerate it well and because extubation from IMV may be difficult.

- In patients with NMD or CWD, deterioration may be rapid or sudden, making HDU/ICU placement for therapy more appropriate.

- In patients with NMD or CWD, senior/experienced input is needed in care planning and is essential if differences in opinion exist or develop between medical staff and patient representatives.

- In patients with NMD, it should be anticipated that bulbar dysfunction and communication difficulties, if present, will make NIV delivery difficult, and may make it impossible.

- Discussion about NIV and IMV, and patients' wishes with respect to cardiopulmonary resuscitation, should occur as part of routine care of patients with NMD or CWD.

- In patients with NMD or CWD, nocturnal NIV should usually be continued following an episode of AHRF, pending discussion with a home ventilation service.

\section{NIV failure and discontinuing NIV following recovery in NMD and CWD \\ Good practice points}

- In patients with NMD or CWD, intolerance of the mask and severe dyspnoea are less likely to cause NIV failure. Bulbar dysfunction makes NIV failure more likely.

- Deterioration in patients with NMD or CWD may be very sudden. Difficulty achieving adequate oxygenation or rapid desaturation during a break from NIV are important warning signs.

- In patients with NMD or CWD, the presence of bulbar dysfunction, more profound hypoxaemia or rapid desaturation during NIV breaks, suggests that placement in HDU/ICU is indicated.

\section{IMV in NMD/CWD}

\section{Recommendations}

54. In patients with NMD or CWD, senior staff should be involved in decision-making, in conjunction with home mechanical ventilation specialists, if experience is limited, and especially when the appropriateness of IMV is questioned (Grade D).

55. Advance care planning, particularly around the potential future use of IMV, is recommended in patients with progressive NMD or CWD. This may best be supported by elective referral to a home ventilation service (Grade D).

\section{IMV strategy in NMD and CWD}

Good practice points

- Patients with NMD usually require low levels of PS.

- Patients with chest wall deformity usually require higher levels of PS.
- PEEP in the range of $5-10$ is commonly required to increase residual volume and reduce oxygen dependency in both patient groups.

\section{Obesity hypoventilation syndrome}

Recommendations

56. Controlled oxygen therapy should be used in patients with OHS and AHRF (Grade D).

57. In patients with OHS, NIV should be started in AHRF using the same criteria as in AECOPD (Grade B).

58. NIV is indicated in some hospitalised obese hypercapnic patients with daytime somnolence, sleep disordered breathing and/or right heart failure in the absence of acidosis (Grade D).

\section{NIV settings and placement in OHS}

Good practice points

- High inspiratory positive airway pressure (IPAP) and expiratory positive airway pressure (EPAP) settings are commonly required in patients with OHS (eg, IPAP $>30, \mathrm{EPAP}>8$ ).

- Volume control (or volume assured) modes of providing NIV may be more effective when high inflation pressures are required.

\section{NIV failure in $\mathrm{OHS}$}

Good practice points

- Fluid overload commonly contributes to ventilatory failure in patients with OHS, and its degree is easily underestimated.

- Forced diuresis may be useful.

- As the risk of NIV failure is greater, and intubation may be more difficult, placement in HDU/ICU for NIV is recommended.

\section{Discontinuing NIV in OHS}

Good practice points

- NIV can be discontinued, as in patients with AECOPD.

- Many patients with AHRF secondary to OHS will require long-term domiciliary support (CPAP or NIV).

- Following an episode of AHRF referral to a home ventilation service is recommended.

\section{IMV strategy in OHS}

Good practice points

- In patients with OHS, pressure controlled MV is recommended initially.

- In patients with OHS, high PEEP settings may be needed to recruit collapsed lung units and correct hypoxaemia.

- In patients with OHS, a forced diuresis is often indicated.

\section{Weaning from IMV \\ Introduction \\ Recommendations}

59. Treating the precipitant cause of AHRF, normalising $\mathrm{pH}$, correcting chronic hypercapnia and addressing fluid overload should all occur before weaning is started (Grade D).

60. A brain natriuretic peptide (BNP)-directed fluid management strategy should be considered in patients with known left ventricular dysfunction. (Grade B)

\section{Weaning methods}

Recommendations

61. Assessment of the readiness for weaning should be undertaken daily (Grade C).

62. A switch from controlled to assisted IMV should be made as soon as patient recovery allows (Grade C). 
63. IMV patients should have a documented weaning plan (Grade B).

Assessing readiness for discontinuation of mechanical ventilation Recommendation

64. A 30 min spontaneous breathing trial (SBT) should be used to assess suitability for extubation (Grade B).

65. Factors including upper airway patency, bulbar function, sputum load and cough effectiveness should be considered prior to attempted extubation (Grade D).

\section{Outcome following extubation}

Recommendation

66. Care is needed to identify factors that increase the risk of extubation failure so that additional support, such as NIV or cough assist, can be provided (Grade B).

\section{Weaning protocols}

\section{Recommendations}

67. Although an organised and systematic approach to weaning is desirable, protocols should be used with caution in patients with AHRF (Grade B).

68. The use of computerised weaning cannot be recommended in AHRF (Grade D).

\section{Use of NIV in the ICU}

\section{Planned NIV to speed weaning from IMV}

Recommendation

69. NIV is recommended to aid weaning from IMV in patients with AHRF secondary to COPD (Grade B).

70. In other causes of AHRF, NIV may have a role in shortening the duration of IMV when local expertise in its use exists (and of cough assist when indicated) and there are features present that indicate extubation is likely to be successful (Grade D).

\section{NIV in high-risk patients}

\section{Recommendation}

71. Prophylactic use of NIV should be considered to provide post-extubation support in patients with identified risk factors for extubation failure (Grade B).

\section{NIV as 'rescue' therapy post-extubation} Recommendations

72. NIV should not be used routinely for unexpected postextubation respiratory failure (Grade B).

73. In COPD, a trial of NIV may be justified for unexpected post-extubation respiratory failure where local expertise exists (Grade D).

\section{Care planning and delivery of care}

Appropriate care environments for the delivery of NIV

Recommendations

74. NIV services should operate under a single clinical lead having formal working links with the ICU (Grade D).

75. The severity of AHRF, and evidence of other organ dysfunction, should influence the choice of care environment (Grade C).

76. NIV should take place in a clinical environment with enhanced nursing and monitoring facilities that are beyond those of a general medical ward (Grade C).

77. Initial care plans should include robust arrangements for escalation, anticipating that around $20 \%$ of AHRF cases should be managed in a level 2 or 3 environment (Grade C).
Good practice points

- A 2-4 bedded designated NIV unit (located within a medical high dependency area or within a respiratory ward with enhanced staffing levels) provides a sound basis for the provision of NIV in a DGH serving a population of 250000 and with an average prevalence of COPD.

- Areas providing NIV should have a process for audit and interdisciplinary communication.

\section{Palliative care and advanced care planning}

\section{Recommendations}

78. Clinicians delivering NIV or IMV should have ready access to palliative medicine (Grade D).

79. Multidisciplinary advance care planning should be an integral part of the routine outpatient management of progressive or advanced disease and care plans should be reviewed on presentation during an episode of AHRF (Grade D).

80. The use of NIV may allow time to establish patient preference with regard to escalation to IMV. (Grade D)

End of life care

Good practice points

- Although removal of the NIV mask may be agreed as preferable, a dignified and comfortable death is possible with it in place.

- Clinicians delivering NIV or IMV should have training in end-of-life care and the support of palliative care teams.

\section{Novel therapies}

Extracorporeal $\mathrm{CO}_{2}$ removal $\left(\mathrm{ECCO}_{2} \mathrm{R}\right)$

Recommendations

81. If local expertise exists, $\mathrm{ECCO}_{2} \mathrm{R}$ might be considered:

- If, despite attempts to optimise IMV using lung protective strategies, severe hypercapnic acidosis $(\mathrm{pH}<7.15)$ persists (Grade D);

- When 'lung protective ventilation' is needed but hypercapnia is contraindicated, for example, in patients with coexistent brain injury (Grade D);

- For IMV patients awaiting a lung transplant (Grade D).

Good practice point

$\mathrm{ECCO}_{2} \mathrm{R}$ is an experimental therapy and should only be used by specialist intensive care teams trained in its use, and where additional governance arrangements are in place, or in the setting of a research trial.

Helium/oxygen ventilation

Recommendation

82. Heliox should not be used routinely in the management of AHRF (Grade B).

\section{ABBREVIATIONS AND GLOSSARY}

ABG Arterial blood gases

AECOPD Acute exacerbation of COPD

AHRF Acute hypercapnic respiratory failure

APACHE II Acute Physiology and Chronic Health

Evaluation: a severity of illness score

ARDS Acute Respiratory Distress Syndrome

Bi-level/Bi-PAP Ventilation mode using 2 levels of pressure support

BMI Body mass index

BODE Body mass index, obstruction, dyspnoea and exercise tolerance score

Bpm Heart rate (beats per minute)

BTS British Thoracic Society 


\begin{tabular}{|c|c|}
\hline $\mathrm{CF}$ & Cystic fibrosis \\
\hline COPD & Chronic obstructive pulmonary disease \\
\hline CPAP & Continuous positive airways pressure \\
\hline CWD & Chest Wall Disease \\
\hline DECAF & $\begin{array}{l}\text { Dyspnoea, Eosinopenia, Consolidation, } \\
\text { Acidaemia and atrial Fibrillation Score }\end{array}$ \\
\hline $\mathrm{ECCO}_{2} \mathrm{R}$ & Extra corporeal carbon dioxide removal \\
\hline ECG & Electrocardiogram \\
\hline EELV & End expiratory lung volume \\
\hline EPAP & Expiratory positive airway pressure \\
\hline ePEEP & Extrinsic PEEP \\
\hline $\begin{array}{l}\text { Expiratory } \\
\text { trigger }\end{array}$ & $\begin{array}{l}\text { Mechanism by which ventilator senses end of } \\
\text { inspiration }\end{array}$ \\
\hline FBC & Full blood count \\
\hline FFM & Full face mask \\
\hline $\mathrm{FiO} 2$ & Fractional inspired concentration of oxygen \\
\hline FRC & Functional residual capacity \\
\hline HDU & High Dependency Unit \\
\hline ICS & Intensive Care Society \\
\hline ICU & Intensive Care Unit \\
\hline IE Ratio & Inspiratory/expiratory time ratio \\
\hline IMV & Invasive mechanical ventilation \\
\hline IPAP & Inspiratory positive airway pressure \\
\hline iPEEP & Intrinsic PEEP \\
\hline $\mathrm{L} / \mathrm{min}$ & Litres per minute \\
\hline NAVA & Neurally adjusted ventilatory assist \\
\hline MND & Motor neurone disease \\
\hline NCROP & $\begin{array}{l}\text { National Chronic Obstructive Pulmonary } \\
\text { Ddisease Resources and Outcomes Project }\end{array}$ \\
\hline NIV & Non-invasive (positive pressure) ventilation \\
\hline NMD & Neuromuscular disease \\
\hline OHS & Obesity hypoventilation syndrome \\
\hline OSA & Obstructive sleep apnoea \\
\hline PAV & Proportional assist ventilation \\
\hline $\mathrm{pCO} 2 / \mathrm{pO} 2$ & Partial pressure of carbon dioxide/oxygen \\
\hline PCV & Pressure controlled ventilation \\
\hline PEEP & Positive end expiratory pressure \\
\hline ePEEP & Extrinsic PEEP \\
\hline iPEEP & Intrinsic PEEP \\
\hline $\mathrm{pH}$ & Acid base balance \\
\hline QoL & Quality of life \\
\hline RCT & Randomised controlled trial \\
\hline $\mathrm{RR}$ & Respiratory rate \\
\hline SBT & Spontaneous breathing trial \\
\hline $\mathrm{SaO} 2$ & Oxygen saturation \\
\hline $\mathrm{TcpCO} 2$ & Transcutaneous measurement of pCO2 \\
\hline $\mathrm{Te}$ & Expiratory duration (seconds) \\
\hline $\mathrm{Ti}$ & Inspiratory duration (seconds) \\
\hline U\&E & Blood urea and electrolyte values \\
\hline VAP & Ventilator associated pneumonia \\
\hline VC & Vital capacity \\
\hline $\mathrm{Vt}$ & Tidal volume \\
\hline
\end{tabular}

\section{INTRODUCTION} Background

The British Thoracic Society (BTS) published the guideline, 'The use of non-invasive ventilation in acute respiratory failure', in 2002. ${ }^{1}$ This was in response to trials demonstrating that NIV was an alternative to IMV in life-threatening respiratory acidosis due to AECOPD. The guideline drew attention to evidence that, when NIV was used in the less severely unwell patient, it also limited progression to more severe respiratory failure. ${ }^{2}$ The trial also demonstrated the feasibility, with adequate staff training, of delivering NIV on a general medical or admission ward with enhanced support.

In subsequent years, NIV has been shown to deliver better rather than equivalent outcomes to invasive ventilation in AECOPD (see Management of hypercapnic respiratory failure section). Although the 2002 guideline recognised NIV to be effective in other causes of AHRF, the evidence was, based largely on an extrapolation from its domiciliary use in neuromuscular and CWD. In the intervening years, better evidence has accumulated for the use of NIV in non-COPD disease. Repeated national audits have, however, raised concerns that expected patient benefit is not being delivered, and have pointed to a number of process deficiencies. ${ }^{3-5}$ There is also the risk, in the absence of justifying trial evidence, that the preferred use of NIV in AECOPD might be extended to all hypercapnic patients, irrespective of circumstance or underlying disease process. That this is a real risk might be inferred from the BTS audits where the indication for NIV was not COPD in over $30 \%$ of cases. ${ }^{3} 4$

NIV development in the UK has been largely outside the organisational 'umbrella' of critical care. This may have adversely affected resource allocation and contributed to a lack of integration in NIV and IMV patient pathways. Other unintended consequences might be a restriction on access to invasive ventilation and delay in the development of extended applications of NIV, such as accelerating extubation and its use in the management of post-extubation respiratory failure, in ICUs. ${ }^{6}$ The 'closed unit' approach advocated in critical care may also have made care of the invasively ventilated respiratory patient the preserve of the intensivist. Such specialists may have little experience of the ability of domiciliary NIV to reverse chronic cardiorespiratory failure and this may lead to underestimating survival, particularly in advanced NMD or CWD.

For these varied reasons, the need for up-to-date guidance was acknowledged by BTS and the Intensive Care Society (ICS). The aim of the guideline is to draw attention to the evidence of suboptimal care in AHRF in the UK, provide an overview of the evidence supporting the use of invasive and non-invasive ventilation, encourage better communication between admitting clinicians and critical care services, promote the use of AHRF patient pathways, and improve resourcing, training, outcomes and patient experience for all adults who develop AHRF.

\section{Definition of AHRF}

AHRF results from an inability of the respiratory pump, in concert with the lungs, to provide sufficient alveolar ventilation to maintain a normal arterial $\mathrm{PCO}_{2}$. Co-existent hypoxaemia is usually mild and easily corrected. Conventionally, a $\mathrm{pH}<7.35$ and $\mathrm{PCO}_{2}>6.5 \mathrm{kPa}$ define acute respiratory acidosis and, when persisting after initial medical therapy, have been used as threshold values for considering the use of non-invasive ventilation. More severe degrees of acidosis, such as $\mathrm{pH}<7.25$, have been used as a threshold for considering provision of IMV.

\section{Importance of AHRF}

AHRF complicates around 20\% of acute exacerbations of COPD. $^{2} 7$ It signals advanced disease, a high risk of future hospitalisations and limited long-term prognosis. The median survival following recovery from AHRF was 1 year in a large case series. $^{7}$ Around $12 \%$ of patients with hypercapnic COPD died during the index admission and this increased to $33 \%$ if the respiratory acidosis developed after hospitalisation. In asthma, acute hypercapnia also signals an increased risk of death and an 
increased likelihood of future life-threatening attacks. ${ }^{8}$ The same risks apply to AHRF complicating CF and bronchiectasis, although this has not been formally reported. In the neuromuscular and CWDs, including morbid obesity, respiratory pump failure is often insidious in its onset, but AHRF may be acute and unexpected. Acute on chronic 'decompensated' episodes of AHRF are more common and normally indicate the future need for domiciliary NIV.

\section{Intended use and target audience of the guideline}

A central theme of the guideline is to promote integration in the planning and delivery of NIV and IMV in AHRF. Despite evidence demonstrating the value of non-invasive ventilation in the management of AHRF, its introduction into routine clinical practice in the UK has not delivered the expected patient benefit and it is likely that NIV provision has, inadvertently, reduced access to IMV in AECOPD and the other causes of AHRF. The introduction, in hospitals accepting acute admissions, of an adequately resourced and integrated AHRF patient pathway is strongly recommended in the expectation that this will lead to improved clinical outcomes and patient experiences.

The target audience for the guideline is medical, nursing and physiotherapy staff working in emergency receiving rooms, medical assessment units, admission wards, respiratory wards and in high dependency and critical care units. The guideline applies to adults. For information on NIV in children with neuromuscular weakness, see the BTS guideline Respiratory Management of Children with Neuromuscular Weakness. ${ }^{9}$

\section{Areas not covered by the guideline}

The guideline does not cover the management of AHRF due to cardiac failure, trauma or acute brain injury. The guideline refers to domiciliary NIV but does not aim to provide guidance on this. The use of non-invasive ventilation is more extensively covered than IMV because the evidence and the clinical experience in its use is recent and because the technical aspects concerning IMV are well covered by standard texts.

\section{Units}

Intrathoracic pressure and pressures relating to mechanical ventilation are presented as $\mathrm{cm} \mathrm{H}_{2} \mathrm{O}$. $\mathrm{ABG}$ tensions are presented as $\mathrm{kPa}$.

\section{Guideline group members}

A list of Guideline Group members and BTS Standards of Care Committee members who assisted with the production of the guideline is given in appendix 1 .

The Guideline Group members adhered to the BTS and ICS policies for the Declaration of Interests and, where appropriate, specific relevant interests are declared in appendix 1.

\section{Methods and terminology}

The guideline has been produced according to the BTS Guideline Production manual and adheres to the criteria set out in the AGREE II instrument. ${ }^{1011}$

\section{Clinical questions and literature search}

Clinical questions were gathered in the PICOT (Patient, Intervention, Comparison, Outcome and Time) format to define the scope of the guideline and inform the literature search. Systematic electronic database searches were conducted in order to identify potentially relevant studies for inclusion in the guideline. For each clinical question, the following databases were searched: Ovid MEDLINE (including MEDLINE In-Process),
Ovid EMBASE, EMSCO CINAHL, Ovid PsycINFO and the Cochrane Library (including the Cochrane Database of Systematic Reviews, the Database of Abstracts of Reviews of Effects and the Cochrane Central Register of Controlled Trials).

An initial search was carried out in November 2010, using a combination of indexed and free text terms defining the clinical questions that had been agreed as important in formulating guidelines in AHRF. It was limited to studies after 1990, on adults, in journals published in English and where at least an abstract was available. The searches identified a total of 582 potential papers, which were subsequently supplemented by publications known to members or resulting from additional searches undertaken by the writing groups after 2010. The literature search was run again in September 2013, for relevant publications between 2010 and 2013, yielding a further 308 potentially relevant references. Additional references were subsequently included from personal collections.

\section{Appraisal of the literature}

Appraisal was performed using the criteria stipulated by the AGREE collaboration. Each paper was appraised by at least two reviewers. The writing lead for each section read the title and abstract of papers identified and agreed with at least one member of each writing group on whether such a paper was definitely relevant, possibly relevant or not relevant, to the section. The criteria used were that the paper addressed a clinical question, the study method used was satisfactory and that the paper was available in English.

Full papers were obtained for all relevant or possibly relevant abstracts. Two members for each section independently appraised each paper, using the SIGN critical appraisal checklists. An evidence level was assigned to each study using SIGN methodology (table 2). These evidence levels are shown in the evidence tables presented in the online supplementary appendix 3 .

\section{Considered judgement and grading of recommendations}

The guideline group used the evidence tables to judge the body of evidence and to develop recommendations for this guideline. Where evidence was lacking, expert opinions were obtained by consensus. The following were considered in the grading of the recommendations: the number of studies and number of patients providing evidence, the applicability of such evidence, and whether generalisable to the patient groups in the guideline and to UK practice and the degree of strength as judged by the consistency of evidence obtained to support recommendations.

Table 2 SIGN levels of evidence

$1++\quad$ High-quality meta-analyses, systematic reviews of RCTs, or RCTs with a very low risk of bias

1+ Well-conducted meta-analyses, systematic reviews or RCTs with a low risk of bias

1- Meta-analyses, systematic reviews or RCTs with a high risk of bias

$2++\quad$ High-quality systematic reviews of case control or cohort or studies High-quality case-control or cohort studies with a very low risk of confounding or bias and a high probability that the relationship is causal

2+ Well-conducted case-control or cohort studies with a low risk of confounding or bias and a moderate probability that the relationship is causal

2- Case-control or cohort studies with a high risk of confounding or bias and a significant risk that the relationship is not causal

3 Non-analytic studies, eg, case reports, case series

$4 \quad$ Expert opinion

$\mathrm{RCT}$, randomised controlled trial 
Recommendations were graded from A to D, using the SIGN Grading System (table 2), as indicated by the strength of the evidence as listed in the tables. Important practical points that lack research evidence were highlighted as 'Good Practice Points'.

Good practice points

Recommended best practice based on the clinical experience of the guideline development group.

\section{Drafting the guideline}

The Guideline Group corresponded regularly. The initial meeting took place in October 2009, and subsequent meetings of the full committee occurred in June and November 2010, September 2011, and March and September 2012. Draft documents were reviewed by the BTS Standards of Care Committee at meetings in 2013 and 2014, and a final draft was produced with the help and collaboration of members of the BTS Standards of Care Committee in September 2014 to March 2015. The guideline was made available for public consultation on the BTS website from 7 May to 12 June 2015. The revised document was reviewed by the BTS Standards of Care Committee in September 2015 and final approval for publication was given in November 2015.

\section{PRINCIPLES OF MECHANICAL VENTILATION Modes of mechanical ventilation}

There are two basic modes of providing mechanical ventilation. In volume-targeted, the operator sets the tidal volume to be delivered and the duration of inspiration (Ti). The ventilator generates whatever pressure is necessary to deliver this volume within this time. In pressure-targeted, the operator sets the inspiratory pressure. The volume of air the patient receives is a function of the impedance to inflation of the lungs and chest wall and the inspiratory time. The $\mathrm{Ti}$ should be of sufficient length to achieve an adequate volume and at a frequency that allows the patient time to fully exhale.

The terminology used for pressure-targeted ventilation can cause confusion. In bi-level ventilation, one pressure is set for inspiration (IPAP) and a second pressure for expiration (EPAP). The difference between the two is the level of ventilatory assistance or PS. This mode is most commonly used for NIV. The same term, CPAP with Pressure Support', can be used to describe a mode of invasive ventilation and/or non-invasive ventilation on some ICU ventilators. The operator sets an incremental inspiratory pressure above the CPAP setting rather than setting an absolute level of inspiratory pressure.

Pressure-targeted ventilation has a number of advantages. First, the pressure delivered is constant and this avoids the sudden and uncomfortable pressure increase that occurs with volume control. Second, pressure-targeted ventilation compensates for air leak, ${ }^{12}{ }^{13}$ which is an inevitable consequence of the interfaces used for NIV. Third, positive pressure throughout expiration (EPAP) flushes exhaled $\mathrm{CO}_{2}$ from the mask and distal ventilator tubing, ${ }^{14}{ }^{15}$ aids triggering (see below) and counteracts the tendency for upper airway collapse during expiration. Pressure ventilators have been used in almost all of the randomised controlled trials (RCTs) in AHRF. ${ }^{16}$ In the UK, volume ventilators are rarely employed (outside of specialist centres) and will not be considered further in this guideline.

In the Spontaneous (S) mode (also known as assist mode), the ventilator delivers assisted breaths in response to patient inspiratory effort. If the patient fails to make adequate inspiratory effort, no ventilator support is delivered. By contrast, in the timed (T) mode (also known as control mode), the ventilator delivers breaths at a rate set by the operator regardless of patient inspiratory effort. 'Pressure-controlled ventilation' (PCV) is the term used to describe a mode in which the operator sets the inspiratory pressure, the length of inspiration and the inspiratory rate. In the spontaneous/timed $(\mathrm{S} / \mathrm{T})$ mode (also known as assist control), a backup rate is set by the operator. If the patient's RR is slower than the backup rate, machine-determined breaths will be delivered (ie, controlled ventilation). If the patient breathes faster than the backup rate, no machine determined breaths will be delivered and all breaths will be triggered (or assisted). The proportion of controlled and assisted breaths often varies, depending on the patient's state of alertness and respiratory drive.

Trigger sensitivity refers to the effort required by the patient to initiate, or trigger, the ventilator. The lower the trigger sensitivity, the greater effort the patient needs to make to trigger a supported breath. Different trigger settings may be required for individual causes of AHRF (see Management of hypercapnic respiratory failure section).

$\mathrm{S} / \mathrm{T}$ is the NIV mode most commonly employed in treating AHRF. There have been no trials comparing PS ventilation and PCV in the treatment of AHRF. Bench studies suggest that ventilators designed specifically for NIV have superior performance over standard ICU ventilators used to deliver NIV, particularly in the presence of significant leak. ${ }^{17-22}$ The extent to which individual types of ICU ventilators (set in the NIV mode) can compensate for leak and the adequacy of patient triggering varies. $^{23}$ Generally, ICU ventilators appear more prone to patient-ventilator asynchrony than home care ventilators. ${ }^{24}$

Evidence statement

Most RCTs that demonstrate an advantage to NIV in AHRF have used pressure targeted ventilators (Level 1+).

Recommendation

1. Pressure targeted ventilators are the devices of choice for acute NIV (Grade B).

Good practice points

- Both PS and pressure control modes are effective.

- Only ventilators designed specifically to deliver NIV should be used.

\section{Choice of interface for NIV}

The FFM is the most suitable interface, as mouth breathing predominates in AHRF. To accommodate the natural diversity of the human face, a range of shapes and sizes of FFM should be available. Reported studies suggest that different types of interfaces do not affect outcome, but the trials have been small and comparison of masks has been inadequately powered to detect a difference. $^{25-37}$ The helmet interface, which covers the whole head, is an alternative to an FFM, ${ }^{38-44}$ but triggering is ineffective. Patients may report about noise caused by turbulence within the helmet, ${ }^{45}$ and it is not possible to provide humidified gases because of 'rain out' in the helmet. A mask that covers the whole of the face (including the eyes, but not the ears) is useful when air leak remains excessive or when nasal bridge ulceration develops, ${ }^{46}$ and is sometimes better tolerated by the confused or agitated patient. In those who find the FFM claustrophobic or distressing, experienced practitioners may consider using a nasal mask or nasal pillows. Mouth leak limits the effectiveness of nasal interfaces during sleep and nasal pillows are more easily dislodged than the FFM.

Ventilators designed for NIV usually employ a single lumen circuit whereas IMV ventilators use a dual lumen circuit (separate tubing for inhalation and exhalation). In the former, a mask with an integral exhalation port is commonly used. If not, an exhalation port needs to be inserted into the ventilator circuit close to the mask. A minimum EPAP of $3 \mathrm{~cm}$ is required to vent 
exhaled air. ${ }^{14} 47$ The website http://ersbuyersguide.org/ offers information on NIV interfaces that are currently available.

Evidence statement

An FFM is the interface of choice for general/non-specialist use (Level 4).

\section{Recommendation}

2. An FFM should usually be the first type of interface used (Grade D).

Good practice points

- A range of masks and sizes is required, and staff involved in delivering NIV need training in and experience of using them.

- NIV circuits must allow adequate clearance of exhaled air through an exhalation valve or an integral exhalation port on the mask.

\section{Indications for and contra-indications to NIV in AHRF}

The indication for NIV will vary according to the underlying cause, severity of illness and associated complicating factors. Broad criteria can be used and are summarised in figure 1, and further discussed in Management of hypercapnic respiratory failure section. Severe facial deformity, fixed upper airway obstruction or facial burns, will occasionally make NIV impossible. A number of other contra-indications have been suggested (see figure 1). ${ }^{48}$ These have most often been employed as exclusion criteria in clinical trials rather than being definitively shown to result in a worse outcome. ${ }^{16}$ Some of the criteria have been challenged. For instance, coma has been regarded as an absolute contra-indication, because of its associated loss of airway protection, but Diaz et al $^{49}$ report similar outcomes with NIV in those with a Glasgow Coma Score $<8$ as the outcomes found in more alert patients. Similarly, confusion, agitation and cognitive impairment make NIV more difficult to apply but should not preclude its use.

There is less haemodynamic compromise with NIV than with IMV, and hypotension should rarely preclude using NIV. Significant arrhythmia, especially if causing hypotension, may tip the balance towards preferring intubation as, in these circumstances, cardioversion may be indicated.

An acute pneumothorax should be drained before applying NIV. If it is too small to allow the safe placement of a chest drain (or is suspected to be chronic) NIV may proceed with careful monitoring. Using a lower inflation pressure seems theoretically sensible but is without evidence. If the patient deteriorates, NIV should be discontinued-in case it is contributing to the development of a tension pneumothorax-and an urgent chest radiograph obtained.

Vomiting has been considered a contra-indication. The key issue is whether the NIV mask can be rapidly removed, that is, an assessment of whether the patient can signal the need to vomit. Marked abdominal distension may sometimes precipitate AHRF in individuals at risk, for example in COPD or morbid obesity. Management should then address the underlying cause of abdominal distension and manage the risk of vomiting by inserting a nasogastric tube. Similarly, in the at-risk patient, hypercapnic respiratory failure may complicate the later stages of pregnancy (eg, kyphoscoliosis or muscular dystrophy). NIV is ideally suited to manage this complication. The need for NIV should be electively assessed (by nocturnal monitoring), but mask ventilation can be initiated during delivery should respiratory distress develop in an at-risk patient.

The presence of copious secretions increases the risk of treatment failure ${ }^{50}$ but NIV may also improve the ability to clear secretions and improve alveolar ventilation. ${ }^{51} 52$

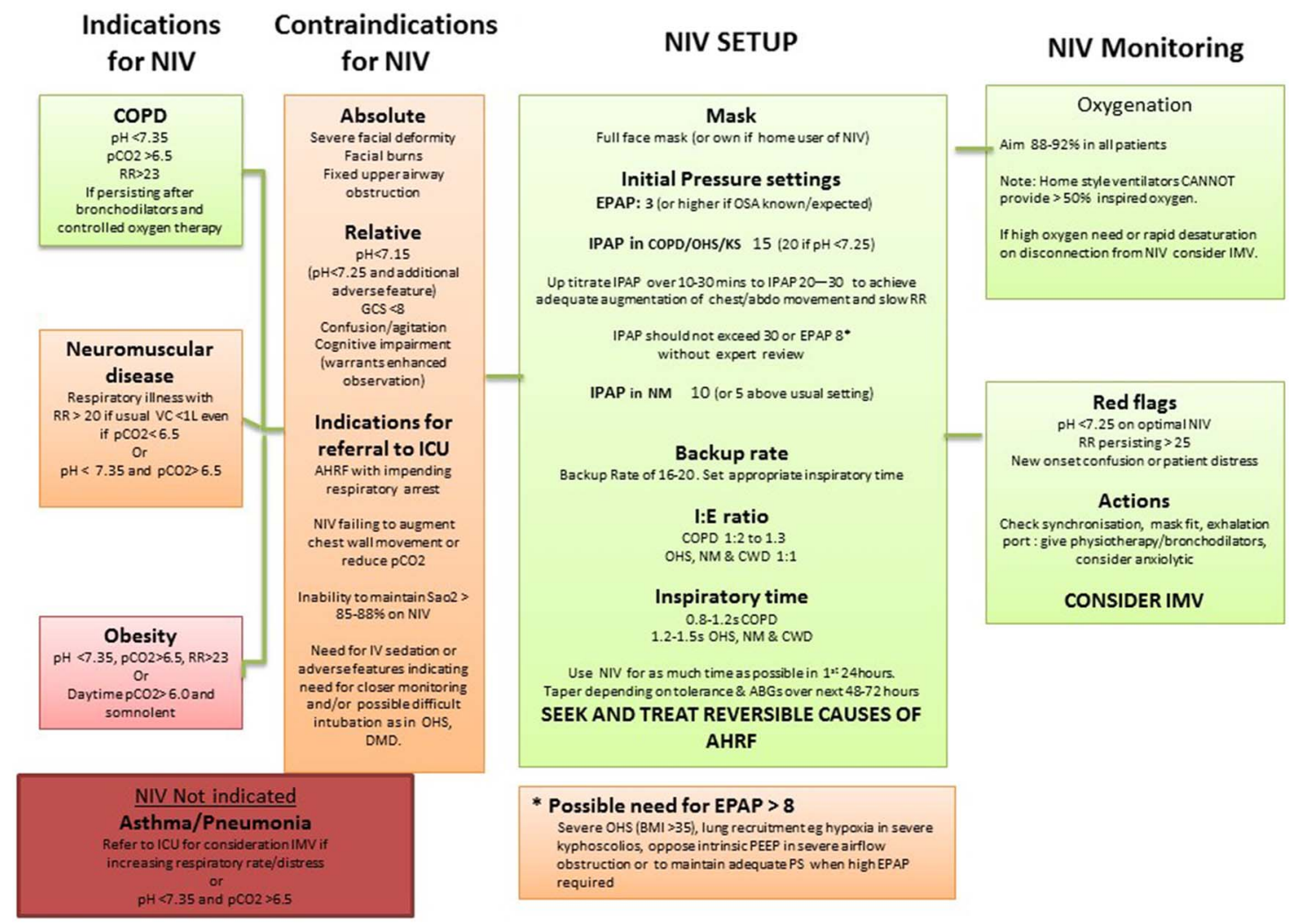

Figure 1 Summary for providing acute non-invasive ventilation. 
Respiratory arrest or peri-arrest have been considered as absolute contra-indications as NIV is intended to supplement spontaneous breathing. However, as bag and mask ventilation (itself a form of NIV) is used as a prelude to intubation, a short trial of NIV by an experienced operator, can be justified while paying special attention to the risk of glottic occlusion.

In summary, the presence of adverse features is an indication for more intense monitoring and placement within HDU/ICU rather than a contra-indication per se.

Evidence statement

There are few absolute contra-indications to a trial of NIV but some adverse features, especially when combined, require more caution and more intense monitoring (Level 4).

The presence of adverse features increases the risk of NIV failure (Level $2++$ ).

\section{Recommendation}

3. The presence of adverse features increases the risk of NIV failure and should prompt consideration of placement in HDU/ ICU (Grade C).

Good practice points

- Adverse features should not, on their own, lead to withholding a trial of NIV.

- The presence of relative contra-indications necessitates a higher level of supervision, consideration of placement in HDU/ICU and an early appraisal of whether to continue NIV or to convert to IMV.

\section{Monitoring during NIV}

Continuous monitoring of oxygen saturation is essential. Repeated measurement of ABG tensions will be required and can be assessed by capillary sampling or intermittent arterial puncture, noting that capillary sampling is less painful for the patient. ${ }^{53}$ One advantage of HDU/ICU placement may be to allow the safe use of an indwelling arterial line for blood sampling. Transcutaneous $\mathrm{pCO}_{2}\left(\mathrm{TcpCO}_{2}\right)$ monitoring is a commonly employed investigation in home ventilation units and the devices are increasingly being employed in hospitals. Small studies have reported on its use in acute respiratory acidosis. ${ }^{55-57} \mathrm{~A}$ study by van Oppen et $a l^{58}$ reported on 10 patients receiving acute NIV and demonstrated that $\mathrm{TcpCO}_{2}$ monitoring is reliable over $12 \mathrm{~h}$ and provides an adequate estimation of $\mathrm{pH}$. Further studies are needed to assess the role of transcutaneous $\mathrm{CO}_{2}$ monitoring.

ECG monitoring is advised for all patients with a tachycardia $>120$ bpm, dysrhythmia or known cardiomyopathy. As in all severely ill patients, serial vital signs (and National Early Warning Scores, where implemented) should be recorded. Good practice points

- Oxygen saturation should be continuously monitored.

- Intermittent measurement of $\mathrm{pCO} 2$ and $\mathrm{pH}$ is required.

- ECG monitoring is advised if the patient has a pulse rate $>120 \mathrm{bpm}$ or if there is dysrhythmia or possible cardiomyopathy.

\section{Supplemental oxygen therapy with NIV}

There are no trials to guide the use of oxygen enrichment. It is well recognised that hyperoxygenation is harmful in the selfventilating patient with AHRF. ${ }^{59-61}$ In the absence of harm from modest hypoxaemia, and to avoid confusion that might arise from having different target saturations in different conditions, a saturation range of $88-92 \%$ is recommended in all patients with AHRF either spontaneously breathing or when receiving NIV. ${ }^{62}$ This is usually easily achieved in AECOPD, but severe hypoxaemia may complicate AHRF in other causative diseases such as CWD.

As for the best method of supplying oxygen, Padkin and Kinnear, ${ }^{63}$ in a study of patients who were not acutely unwell, reported no difference in inspired content whether delivered directly into the NIV mask or into the ventilator tubing close to the mask. Introducing oxygen at the ventilator end of the tubing was less effective. The mean $\mathrm{FiO}_{2}$ achieved was $31 \%$ at $1 \mathrm{~L} / \mathrm{min}$, $37 \%$ at $2 \mathrm{~L} / \mathrm{min}, 40 \%$ at $3 \mathrm{~L} / \mathrm{min}$ and $44 \%$ at $4 \mathrm{~L} / \mathrm{min}$. Flow rates $>4 \mathrm{~L} / \mathrm{min}$ provided minimal additional increase. Kaul ${ }^{64}$ found that the higher the inspiratory pressure, the less additional benefit resulted from higher flow rates (because higher pressures increase leak). High flow rates also resulted in delay triggering the ventilator. As this risks promoting patient ventilator asynchrony, technically advanced NIV ventilators that allow precise oxygen blending (and a higher $\mathrm{FiO}_{2}$ enrichment) are a safer and more appropriate alternative when hypoxaemia is severe.

Evidence statements

In AHRF-targeted oxygen therapy $\left(\mathrm{SaO}_{2} 88-92 \%\right)$ reduces mortality (Level $1+$ ).

When providing NIV, oxygen enrichment is best given at or near the mask (Level 3).

Recommendations

4. Oxygen enrichment should adjusted to achieve $\mathrm{SaO}_{2} 88-$ $92 \%$ in all causes of AHRF being treated by NIV (Grade A).

5. Oxygen should be entrained as close to the patient as possible (Grade C).

Good practice points

- As gas exchange will improve with increased alveolar ventilation, NIV settings should be optimised before increasing the $\mathrm{FiO} 2$.

- The flow rate of supplemental oxygen may need to be increased when ventilatory pressure is increased to maintain the same $\mathrm{SaO} 2$ target.

- Mask leak and delayed triggering may be caused by oxygen flow rates $>4 \mathrm{~L} / \mathrm{min}$, which risks promoting or exacerbating patient-ventilator asynchrony. The requirement for high flow rates should prompt a careful check for patient-ventilator asynchrony.

- A ventilator with an integral oxygen blender is recommended if oxygen at $4 \mathrm{~L} / \mathrm{min}$ fails to maintain $\mathrm{SpO} 2>88 \%$.

\section{Humidification with NIV}

There is no evidence to guide the use of humidification in acute NIV. Heated humidification may reduce upper airway resistance and increase comfort when leak is high. ${ }^{65}$ In short-term studies, heated humidification reduces upper airway dryness, ${ }^{66} 67$ which might improve tolerance and aid secretion clearance, but this has not been proven. Humidification should only be considered when upper airway dryness is a problem or secretions are difficult to expectorate.

Evidence statement

No evidence exists to guide humidification practice in acute NIV (Level 4).

Recommendation

6. Humidification is not routinely required (Grade D).

Good practice point

Heated humidification should be considered if the patient reports mucosal dryness or if respiratory secretions are thick and tenacious. 


\section{Bronchodilator therapy with NIV}

As part of a PhD thesis, Kaul ${ }^{68}$ found that nebulised bronchodilators given concomitantly with NIV in stable patients produced less benefit than when given while patients were breathing spontaneously. Brief discontinuation of NIV for the administration of bronchodilators appears to be safe ${ }^{69}$ Acccordingly, bronchodilator therapy is probably better given during breaks in NIV. This may also facilitate coughing and the clearing of respiratory secretions. If discontinuing NIV results in patient distress, it should be continued and a nebuliser sited proximally in the circuit. $^{70}$

Good practice points

- Nebulised drugs should normally be administered during breaks from NIV.

- If the patient is dependent on NIV, bronchodilator drugs can be given via a nebuliser inserted into the ventilator tubing.

\section{Sedation with NIV}

Patient agitation and distress are common in AHRF and may be made worse by the application of NIV before gas exchange has improved and the patient has sensed a reduction in the work of breathing. Despite this, sedatives/anxiolytics and/or opiates are infrequently used due to concern about depressing respiratory drive. This is understandable if NIV is delivered in an inappropriate environment that is unable to provide continuous monitoring and that does not have the ready availability of medical staff to perform safe intubation if needed. On the contrary, relieving patient distress is an important goal and might be expected to increase comfort and the success of NIV. In a 2007 survey of members of the critical care assemblies of the American College of Chest Physicians and the European Respiratory Society, respondents reported using sedatives or opiates in only $25 \%$ of cases and $21 \%$ stated they had never used either. ${ }^{71}$ The risk of respiratory depression was given as the reason for non-use. Individual practice was highly variable and, as the response rate was poor (42\% European, 14\% North American), the conclusions reported are more qualitative than quantative. When treatment was given it was mostly by bolus injection and rarely according to a sedation protocol. Greater experience in the use of NIV and being a critical care clinician increased reported use of opiates/sedation.

In the 2013 BTS audit, involving 2693 cases, NIV failed to reverse AHRF in $30 \%$ of patients. ${ }^{5}$ Agitation was reported as the principal reason in $31 \%$ of these. Sedation was 'attempted' in $84 \%$. No details are available on what agents were used, or outcome in those so treated. As $91 \%$ of all NIV treatments were provided outside of the HDU/ICU, it appears sedation is now more commonly employed but in a potentially unsafe environment.

In the ICU setting, case series have reported that infusions of propofol, ${ }^{72}$ dexmedetomidine ${ }^{73}$ and remifentanyl ${ }^{74}{ }^{75}$ are safe, improve comfort and reduce the failure rate of NIV. Senoglu et $a l^{76}$ compared infusions of dexmedetomidine and midazolam in 45 AECOPD cases with AHRF, using a protocol aiming at a standard degree of sedation. No differences were found in effectiveness between the two agents. There were no significant adverse events and no patient failed to improve with NIV. In another report, the addition of infused dexmedetomidine to a standard protocol of 'as needed' bolus intravenous midazolam and fentanyl, given according to a sedation protocol, failed to show benefit, but sedation goals were readily achieved and there was good NIV tolerance and success with the standard proto$\mathrm{col}^{77}{ }^{77}$ A review of sedation to facilitate NIV tolerance makes the pharmacological case for preferring an opiate to a benzodiazepine (because the latter promotes upper airway obstruction through inhibiting the pharangeal dilating muscles) but concluded that studies to date have been too small, have used different drugs and therapy regimes and employed a variety of outcome measures. ${ }^{78}$ Guidance on the use of sedation within hospitals might be expected to improve patient safety when implemented. ${ }^{79}$

\section{Evidence statements}

Patient distress is common in AHRF and often made initially worse by applying NIV (Level 4).

There is inadequate evidence to guide the use of sedation/anxiolysis in acute NIV. Their use in a critical care setting is reported to improve outcome and reduce patient distress (Level 2-).

Recommendations

7. Sedation should only be used with close monitoring (Grade D).

8. Infused sedative/anxiolytic drugs should only be used in an HDU or ICU setting (Grade D).

9. If intubation is not intended should NIV fail, then sedation/ anxiolysis is indicated for symptom control in the distressed or agitated patient (Grade D).

Good practice point

In the agitated/distressed and/or tachypnoeic individual on NIV, intravenous morphine $2.5-5 \mathrm{mg}$ ( \pm benzodiazepine) may provide symptom relief and may improve tolerance of NIV.

\section{NIV complications}

The reported rate of complications varies widely. One review gives an incidence between $30 \%$ and $50 \%,{ }^{80}$ the range partly depending on how a complication is defined. Extended duration of NIV, patient agitation and the frequent need to adjust mask fit are all associated with an increase in rate/severity of mask-related problems.

Nasal bridge ulceration is the most common problem $(5-10 \%)$ and may be severe enough to result in NIV failure. ${ }^{81}$ Overtightening is a common cause. NIV masks are designed to mould to the face when pressurised which over-tightening impairs. Should signs of skin trauma become apparent, a barrier dressing and a strategy of regular breaks and alternating between two interface types should be used. Latex allergy occasionally results in florid skin reactions. Some patients seem especially prone to mask-related rash even in the absence of allergy. Topical steroids may be indicated and/or antibiotics if the wound becomes infected.

NIV may cause severe gastric distension. It usually indicates poor coordination between patient and ventilator and it may be necessary to insert a nasogastric tube. Sinus or ear discomfort and nasal mucosal congestion or drying/ulceration can all occur. The value of humidification in preventing these side effects is uncertain but water-based nasal gels and topical corticosteroids or decongestants can be used. Petroleum-based emollients should not be used with supplemental oxygen.

An acute pneumothorax may be life-threatening but difficult to detect. The development of unexplained agitation/distress or chest pain requires this complication to be excluded. ${ }^{82}$ Co-existent interstitial lung disease or previous episodes of spontaneous or ventilator-induced pneumothorax increase the risk. Using a lower IPAP to avoid large tidal volumes, and a lower EPAP to avoid significantly increasing end-expiratory lung volume (EELV), are logical but not evidence based. If a pneumothorax develops, intercostal drainage is usually required. 


\section{Good practice points}

- Minor complications are common but those of a serious nature are rare. Patients should be frequently assessed to identify potential complications of NIV.

- Care is needed to avoid overtightening of masks.

- Previous episodes of ventilator-associated pneumothorax warrant consideration of admission to HDU/ICU and use of NIV at lower than normal inspiratory pressures.

- The development of a pneumothorax usually requires intercostal drainage and review of whether to continue with NIV.

\section{Sputum retention}

Sputum retention can be a precipitant for AHRF, can cause NIV to fail and is a common reason for respiratory distress postextubation in patients initially managed by IMV. Excessive sputum production characterises bronchiectasis and CF, and complicates some patients with AECOPD. Promoting sputum clearance can be particularly challenging in those with NMD and in the morbidly obese. Techniques, such as manually assisted cough and mechanical insufflation-exsufflation (MI-E), aid sputum clearance in patients with NMD. ${ }^{83}$ However, in a study including patients with either scoliosis or COPD, MI-E reportedly had no benefit. ${ }^{85}$ In another RCT, the use of MI-E reduced post-extubation respiratory failure in a mixed group of patients including some with AHRF. ${ }^{86}$ This study also provided NIV to those in respiratory distress. The reader is referred to the BTS Physiotherapy Guidelines ${ }^{87}$ for more detailed information.

Mini-tracheostomy facilitates secretion clearance in the spontaneously breathing patient ${ }^{88}$ and may have a role when sputum retention is thought to be a major determinant of AHRF, such as in CF. It is not an easy technique to perform in the anxious and breathless patient and training opportunites are rare. Clinicians who insert percutaneous tracheostomies are best placed to provide a service and the HDU/ICU is the best environment in which to perform mini-tracheostomy. In an attempt to avoid intubation, a combination of respiratory support by NIV and suctioning via a mini-tracheostomy has been described. This probably only has application if IMV is not desired by the patient as, in most such cases, IMV offers more chance of a successful outcome. In the patient initially managed by IMV, a mini-tracheostomy may be inserted at the time of endotracheal tube decannulation in patients with a high secretion load and/or a poor cough.

\section{Evidence statements}

Manual-assisted cough and MI-E are safe methods for aiding secretion clearance (Level 1+).

MI-E is more effective than manual-assisted cough in patients with stable NMD (Level 2+).

Mini-tracheostomy is a useful bedside procedure that can markedly improve secretion clearance, but requires patient cooperation and a skilled operator to be performed safely (Level 4).

\section{Recommendations}

10. In patients with NMD, mechanical insufflation and exsufflation should be used, in addition to standard physiotherapy techniques, when cough is ineffective and there is sputum retention (Grade B).

11. Mini-tracheostomy may have a role in aiding secretion clearance in cases of weak cough (NMD/CWD) or excessive amounts (COPD, CF), (Grade D).

\section{Modes of IMV}

Critical care ventilators are complex devices capable of delivering multiple modes. ${ }^{89}$ The traditional divide between pressure and volume has become blurred and hybrid modes combine aspects of both. Most patients with AHRF do not require sophisticated modes of providing IMV.

Initially, when airway resistance is high and/or compliance is low (eg, in asthma, CF or bronchiectasis) a period of mandated or 'controlled mechanical ventilation', often combined with deep sedation to reduce spontaneous breathing effort, allows time for bronchodilators, steroids and antibiotics to treat airway inflammation, overcome infection and for 'bronchial toilet' to be provided. These considerations also variably apply to the restrictive causes of AHRF. In addition, poor triggering, because of muscular weakness, is a risk in patients with NMD in whom a prolonged period of controlled mechinical ventilation may be necessary. In all patients with AHRF, allowing restorative sleep is important. ${ }^{91-93}$

Management should shift towards supporting rather than mandating the pattern of ventilation as recovery begins. If there is adequate spontaneous effort, and the RR is not excessive, a switch to PS is recommended to reduce the need for sedation and also as the risk of respiratory muscle wasting may be reduced by establishing early spontaneous breathing. The concept that suppressing spontaneous breathing is causally related to diaphragm wasting is contentious in the literature. Space constraints prevent a fuller examination. One initially compelling human study that claimed to have demonstrated 'disuse atrophy' was subsequently critised because the diaphragms had been denervated. ${ }^{94}$ A study in patients with adult respiratory distress syndrome (ARDS) reported that those patients allowed to breathe spontaneously had less need for sedation than patients treated with controlled IMV, a reduced requirement for vasopressors, fewer days of ventilatory support, earlier extubation and a shorter length of ICU stay. ${ }^{95}$ This strategy has not been assessed in AHRF.

Evidence statements

Establishing early spontaneous breathing reduces the need for sedation, improves cardiac function and reduces the duration of IMV in ARDS (Level 1-).

\section{Recommendations}

12. Spontaneous breathing should be established as soon as possible in all causes of AHRF (Grade C).

13. Controlled IMV may need to be continued in some patients due to severe airflow obstruction, weak muscles leading to poor triggering or to correct chronic hypercapnia (Grade C).

Good practice point

In obstructive diseases, controlled IMV should be continued until airway resistance falls.

\section{Invasive ventilation strategy}

In obstructive causes, tidal volume $(\mathrm{Vt})$ is limited by the airflow obstruction and compounded by the mechanical disadvantage of hyperinflation. The use of high inflation pressures, to achieve a 'normal' Vt, risks dynamic hyperinflation. ${ }^{96}$ It most dramatically occurs soon after intubation but may develop on switching ventilation mode, for example, from controlled to assisted ventilation. ${ }^{97}$

The adverse consequences of hyperinflation include barotrauma, impaired gas exchange and patient discomfort. The increased intrathoracic pressure impedes venous return and increases right ventricular afterload with a resulting fall in cardiac output and hypotension. ${ }^{98}$

Prolonging expiratory time limits gas trapping and is achieved by shortening the inspiratory time and reducing the minute volume, an approach recommended in airflow obstruction. ${ }^{99} 100$ If significant gas trapping still occurs, the recommendation is to use a lower than normal $\mathrm{Vt}$ in combination with a low RR and 
Figure 2 Guide to initial settings and aims with invasive mechanical ventilation.
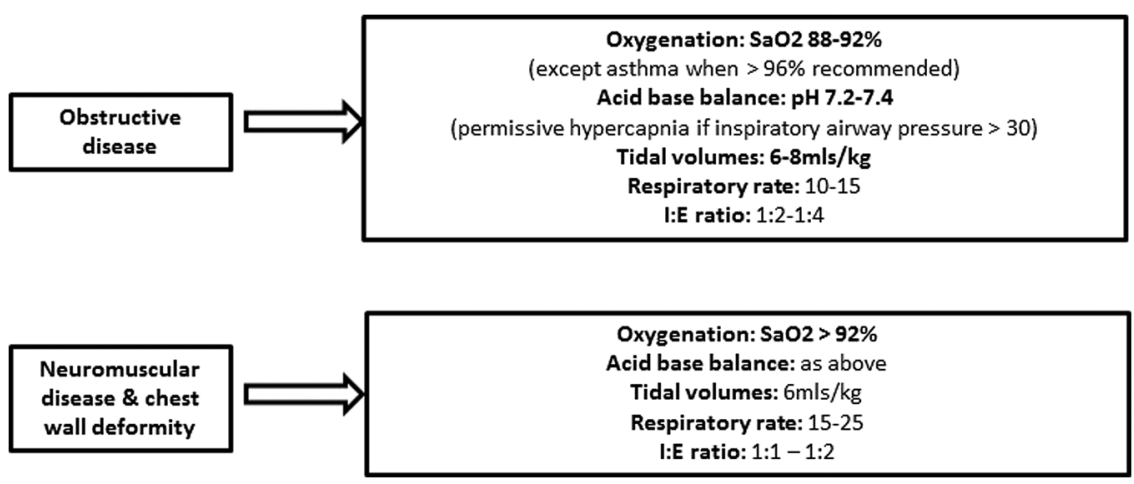

a more prolonged expiratory phase. ${ }^{99} 100$ This can often only be achieved using a controlled ventilation mode combined with deeper levels of sedation. On switching to PS (assist) during recovery, the inspiratory pressure needs to be sufficient to provide adequate tidal volume but not excessive. Settings therefore need to be individually adjusted and require regular review.

In ARDS, over-distention and repetitive recruitment/ de-recruitment of lung units causes alveolar damage (so-called ventilator-induced lung injury) and may even provoke systemic inflammation. ${ }^{101}$ One explanation for improved outcome with low $\mathrm{Vt}$ ventilation $(<6 \mathrm{~mL} / \mathrm{kg})$, compared with conventional practice, may be avoidance of ventilator-induced lung injury. ${ }^{102}$ The ARDS literature provides evidence for permissive hypercapnia, demonstrating that a $\mathrm{pH}$ above 7.2 is well tolerated. ${ }^{103}$ This is the consensus target when $\mathrm{pH}$ control is difficult. ${ }^{89} 90$ Allowing permissive hypercapnia will result in cerebral vasodilation and a rise in intracranial pressure and may also compromise myocardial contractility. Attempts to raise $\mathrm{pH}$ to $>7.2$ may, however, compound hyperinflation and barotrauma. In ARDS, a peak airway pressure of $30 \mathrm{~cm}$ is the usual trigger for employing permissive hypercapnia, a strategy that reduces mortality. ${ }^{104}$

In AECOPD, attempts to rapidly restore $\mathrm{pO}_{2}$ and $\mathrm{pCO}_{2}$ to normal are unnecessary. Although there is little evidence to provide guidance, it is suggested that the higher the pre-morbid $\mathrm{pCO}_{2}$ (inferred by a high admission bicarbonate), the higher the target $\mathrm{pCO}_{2}$ should be. Recovery from extreme levels of hypercapnia is recognised. ${ }^{105}$ Any metabolic causes of acidosis, for example, from insulin insensitivity or excessive B2 stimulated glycogenolysis, should be treated separately.

In NMD, an adequate tidal volume can be achieved with relatively low inflation pressures (eg, 10-15), but higher pressure is needed in CWD because of reduced chest wall compliance. Lung recruitment strategies (ie, increasing PEEP) should be considered when there is persisting hypoxia and/or evidence of premature small airway closure in dependent lung tissue. Controlled MV may need to be continued in NMD when triggering is likely to be inadequate or tiring.

Reducing the bicarbonate buffering capacity will require a period of relative hyperventilation when hypercapnia is chronic. The resulting urinary bicarbonate loss resets central respiratory drive. Carbonic anhydrase inhibitors can be used but caution is needed as high doses produce unpredictable effects through central stimulation of breathing. ${ }^{106} 107$

Evidence statements

In ARDS, a low Vt strategy improves survival (Level 1+).

In airflow obstruction, prolonging the expiratory time reduces dynamic hyperinflation (gas-trapping) (Level 2+).
Recommendations for IMV in obstructive disease

14. During controlled ventilation, dynamic hyperinflation should be minimised by prolonging expiratory time (I:E ratio $1: 3$ or greater) and setting a low frequency (10-15 breaths/min) (Grade C).

15. Permissive hypercapnia (aiming for $\mathrm{pH}$ 7.2-7.25) may be required to avoid high airway pressures when airflow obstruction is severe (Grade D).

16. Carbonic anhydrase inhibitors should not be used routinely in AHRF. (Grade C).

\section{Positive end expiratory pressure}

PEEP is an area of physiology that causes confusion among healthcare professionals. The best way to set optimal PEEP remains contentious. Simply stated, PEEP shifts the lungs to a more compliant portion of the pressure-volume curve. In restrictive causes of AHRF, lung volume is usually reduced and there may be dependent lung that is poorly ventilated or in which there is no effective alveolar ventilation. In these circumstances, increasing external PEEP increases $\mathrm{Vt}$ for a given inspiratory pressure, will reduce $\mathrm{pCO}_{2}$ and improve oxygenation. In obstructive disease, PEEP improves expiratory airflow, limits dynamic hyperinflation and improves alveolar ventilation. ${ }^{108} 109$ Dynamic hyperinflation may be suspected by a progressive fall in tidal volume with constant ventilator pressure settings (or, with volume control, an increase in inflation pressure) and by signs of increasing patient distress such as tachycardia and hypotension.

The degree of intrinsic PEEP (iPEEP) can be estimated by examination of the expiratory flow curve and pressure ${ }^{110}$ or be measured invasively. ${ }^{111} 112$ Active expiratory muscle contraction, common in airflow obstruction, will artificially increase apparent iPEEP. Levels of iPEEP in obstructive airways disease have been reported to range from 4.6 to $13.6 \mathrm{~cm} \mathrm{H}_{2} \mathrm{O}^{113}$

Setting the PEEP level in excess of iPEEP may be deleterious. This has led to the recommendation that PEEP be set at 50 $80 \%$ of iPEEP. ${ }^{114} 115$ However, as the severity of airway obstruction in small airways will vary throughout the lung, a variable response to increasing the PEEP might be anticipated. If, on balance, an increase in ePEEP were to reduce overall airway resistance then EELV will fall even though ePEEP apparently exceeds iPEEP. ${ }^{116} 117$

Intrinsic PEEP is a pressure that must be overcome by patient effort before a breath can be triggered. It is, therefore, an inspiratory threshold load and may lead to ineffective triggering and patient discomfort. Offsetting iPEEP by increasing the ventilator PEEP will then reduce the effort of triggering and 
improve patient-ventilator asynchrony. ${ }^{118-120}$ It is important to appreciate that the same pathophysiological processes occur during treatment with NIV when a higher EPAP setting may improve triggering, patient comfort and oxygenation.

\section{Evidence statement}

In obstructive causes of AHRF, PEEP may increase tidal volume, improve compliance and reduce airflow obstruction (Level $2+$ ). Setting PEEP greater than iPEEP can be harmful (Level $2+$ ).

In restrictive causes of AHRF, PEEP may assist in lung recruitment, improve compliance and correct hypoxaemia (Level 3).

\section{Recommendation}

17. Applied ePEEP should not normally exceed $12 \mathrm{~cm}$ (Grade C).

\section{Sedation in IMV}

Patients receiving IMV require sedation, especially before stability is achieved. ${ }^{89}$ Most ICUs use Propofol or a benzodiazepine, either alone or in combination with an opioid. Benzodiazepines with inactive metabolites and/or short acting synthetic opioids have been recommended to avoid over-sedation. ${ }^{121} 122$ Although sedation increases IMV tolerance, over-use is associated with adverse outcomes such as prolonged duration of IMV, increased ICU length of stay and delirium. ${ }^{123}$

To avoid this, withholding of further sedation until an objective degree of wakefulness develops has been investigated. In two trials, this strategy was shown to reduce duration of IMV and ICU length of stay. ${ }^{124} 125$ Studies employing sedation protocols targeting specific (higher) levels of alertness have also reported a reduction in duration of IMV, ICU and hospital length of stay. $^{126-129}$ However, a meta-analysis of RCTs on sedation breaks demonstrated safety but failed to confirm benefit, ${ }^{130}$ and a more contemporary RCT, combining protocolised sedation with daily breaks, also found no benefit. ${ }^{131}$ No study has shown harm from sedation breaks. The effect of stopping or reducing sedation on patient experience has not been reported.

Evidence statements

Daily interruption of sedation is safe and may reduce the duration of IMV and ICU length of stay (Level 1+).

Sedation protocols that target specific levels of alertness may reduce duration of IMV and ICU length of stay (Level 1+).

\section{Recommendation}

18. Sedation should be titrated to a specific level of alertness (Grade B).

\section{Patient-ventilator asynchrony}

Patient-ventilator asynchrony is common and increases patient discomfort, the work of breathing, the need for sedation, the incidence of confusion, the need for tracheostomy and the mortality rate. ${ }^{132} 133$ The commonest cause is ineffective triggering due to either respiratory muscle weakness and/or excessive effort required to overcome iPEEP and trigger a breath. ${ }^{134}$ Trigger failure is more common during sleep and more likely if hypercapnia persists by day. A hybrid mode, such as PS with a mandatory backup rate is recommended in these circumstances to avoid pCO2 increasing during sleep.

Auto triggering refers to inappropriately delivered breaths being provided by the ventilator. It can be provoked by patient movement, suctioning, coughing and swallowing, and is more likely when the trigger sensitivity is set too high. Both a delay in the onset of a triggered breath or an inadequate amount of PS to sufficiently augment inspiratory flow can lead to an unpleasant sensation best described as 'air hunger'. This can be difficult to detect or for the patient to report. Experienced NIV practitioners may trial increasing trigger sensitivity and/or PS, and monitor the effect on patient comfort and RR. If inadequate PS is given, the breathing rate will fall. The detection of the more subtle forms of patient-ventilator asynchrony requires examination of the pressure/flow waveforms. ${ }^{135}$ The most sensitive measure of patient-ventilator asynchrony is by simultaneous recordings of diaphragm electrical activity and pressure changes in the oesophagus. ${ }^{134}$ Flow rather than pressure triggers reduce the incidence of asynchrony, ${ }^{136} 137$ as has the move away from volume-controlled ventilation. ${ }^{138} 139$

Proportional assist ventilation (PAV) and neurally adjusted ventilatory assist (NAVA) are modes that are being assessed as ways to reduce patient-ventilator asynchrony. With PAV, the degree of pressure support is determined, on a breath by breath basis, by the patient's inspiratory effort. ${ }^{140-142}$ Compared with PS, PAV has been reported to reduce the probability of returning to a controlled mode and the incidence of patient-ventilator asynchrony. ${ }^{143}$ In NAVA, the ventilator attempts to match neural drive by adjusting the degree of PS (within safe limits), using the electrical activity of the diaphragm to 'drive' the ventilator. Studies comparing patient-ventilator interaction show a reduction in triggering delay with NAVA, reduced cycling delay and a reduction in asynchrony events. ${ }^{144} 145$ Uncertainties persist on how to adjust the NAVA level and this technical issue is currently frustrating efforts to demonstrate clinical benefit.

It is important to emphasise that patient ventilator asynchrony is common with NIV. While the same principles apply it has been less frequently recognised or investigated. It can critically affect the success of NIV and the patient experience (see below). Evidence statements

Patient-ventilator asynchrony is common and deleterious, and can be minimised through informed adjustment of ventilator settings (Level 2+).

Proportional and NAVA have been shown experimentally to reduce ventilator asynchrony but have yet to improve patient outcome (Level 2+).

Recommendations

19. Ventilator asynchrony should be considered in all agitated patients (including NIV) (Grade C).

20. As patients recover from AHRF, ventilator requirements change and ventilator settings should be reviewed regularly (Grade C).

\section{Use and timing of a tracheostomy}

It is accepted that translaryngeal intubation beyond 10 days can be detrimental. ${ }^{146} 147$ Historically, it was believed that early tracheostomy reduced ventilator time and ICU length of stay. ${ }^{148}$ A survey of ICU physicians in 2005 found that $61 \%$ of respondents would perform a tracheostomy without first performing a trial of extubation and 50\% favoured tracheostomy insertion within the first week. ${ }^{149}$ Two large multicentre studies have failed to show benefit from tracheostomy performed within 7 days of admission. ${ }^{150}{ }^{151}$ A subsequent meta-analysis also reported no effect on the incidence of ventilator-associated pneumonia or mortality, ${ }^{152}$ although less sedation was required after a tracheostomy had been inserted. Tracheostomy carries a morbidity and mortality risk at the time of insertion ${ }^{153}$ and subsequently. ${ }^{154}$ A UK national report has highlighted the risk of critical airway incidents in patients with tracheostomies. ${ }^{155}$ Accordingly, consideration of the risk and benefit should be undertaken before proceeding to insert a tracheostomy and due consideration should be given to using NIV post-extubation to avoid a tracheostomy. This is particularly the case in progressive $\mathrm{NMD} / \mathrm{CWD}$ when tracheostomy insertion carries the risk of permanence. These aspects, and the evidence summarised 
below, are considered further in Management of hypercapnic respiratory failure section.

Evidence statement

Early insertion of a tracheostomy does not reduce mortality, duration of IMV, or the incidence of ventilator-associated pneumonia (Level $1++$ ).

\section{Recommendations}

21. Performing routine tracheostomy within 7 days of initiating IMV is not recommended (Grade A).

22. The need for and timing of a tracheostomy should be individualised (Grade D).

Good practice points

- In AHRF due to COPD, and in many patients with NMD or OHS, NIV-supported extubation should be employed in preference to inserting a tracheostomy.

- In AHRF due to NMD, alongside discussion with the patient and carers, the decision to perform tracheostomy should be multidisciplinary and should involve discussion with a home ventilation unit.

\section{MANAGEMENT OF AHRF \\ Obstructive lung diseases}

Acute exacerbations of COPD account for 100000 admissions annually in England. Of these, around 20\% will present with or develop hypercapnia, ${ }^{2} 7$ an indicator of increased risk of death. $^{2} 59$ The development of AHRF is often multifactorial. These include infection, mucosal oedema, bronchospasm, sputum retention, excessive $\mathrm{O}_{2}$ therapy, sedation, pneumothorax, PE and left ventricular failure. Since the publication of the BTS guideline in $2002^{1}$ and subsequent National Institute for Health and Care Excellence (NICE) recommendations, ${ }^{48}$ the use of NIV in AECOPD has increased and most hospitals admitting unselected medical patients are able to provide an NIV service. ${ }^{60}$

\section{Prevention of AHRF in AECOPD}

There is compelling evidence that uncontrolled oxygen therapy increases the degree of acidosis and subsequent mortality in AECOPD. ${ }^{2}{ }^{156}$ In a trial comparing the use of high concentration oxygen versus titrated oxygen in 405 individuals with presumed AECOPD in the pre-hospital (ambulance/paramedic) setting, Austin et $a l^{61}$ reported that titrated oxygen reduced mortality by $58 \%$ for all patients (relative risk 0.4 ) and by $78 \%$ for patients with confirmed COPD (RR 0.22). Patients with COPD who had received titrated oxygen according to the protocol (targeted at a saturation of $88-92 \%$ ) were less likely to have respiratory acidosis (mean difference in $\mathrm{pH}$ 0.12) than those who received high-concentration oxygen. These data provide further evidence to titrate oxygen treatment to a modest target saturation in patients with breathlessness and a history or clinical likelihood of COPD. Importantly, the mechanism(s) of oxygen-induced hypercapnia apply, to varying degree, in the other causes of AHRF. Accordingly, the same oxygen saturation target is recommended in the initial management of all patients at risk of AHRF.

Evidence statement

The use of controlled oxygen therapy in individuals with suspected AECOPD reduces mortality and the frequency and severity of AHRF (Level $1++$ ).

Recommendation

23. In AHRF due to AECOPD, controlled oxygen therapy should be used to achieve a target saturation of $88-92 \%$ (Grade A).
Good practice point

Controlled oxygen therapy should be used to achieve a target saturation of $88-92 \%$ in ALL causes of AHRF.

\section{Role of NIV in AECOPD}

There are three clinical situations in which NIV is recommended in AECOPD ${ }^{157}$ First, the patient with a modest respiratory acidosis with the aim of preventing deterioration to a point when IMV would conventionally be considered. Second, as an alternative to IMV when conventional criteria for IMV are met (lower $\mathrm{pH}$, more distress) with the intention to proceed to IMV if NIV fails. Third, as the 'ceiling' of treatment for patients who, for whatever valid reason, are not candidates for IMV. The evidence base for NIV has rarely defined the particular patient case mix in this way.

There have been many trials of NIV in acidotic AECOPD, including 21 where NIV was compared to standard nonventilatory treatment, one trial of NIV versus sham NIV and two trials of NIV versus IMV. Five of the studies were conducted in an ICU setting, one in the pre-hospital setting, two in emergency departments (EDs), two in HDUs and the remainder in general wards. In general, patients in studies conducted in the ICU had lower $\mathrm{pH}$ and therefore more severe exacerbations. ${ }^{158}$

In a meta-analysis of NIV use versus usual care, NIV was associated with a lower mortality (relative risk 0.41), a lower need for intubation (relative risk 0.42), lower likelihood of treatment failure (relative risk 0.51) greater improvements at $1 \mathrm{~h}$ in $\mathrm{pH}$ (weighted mean difference 0.03 ), $\mathrm{pCO}_{2}$ (weighted mean difference $-0.40 \mathrm{kPa}$ ) and RR (weighted mean difference -3.08 breaths $/ \mathrm{min}$ ). NIV also resulted in fewer treatment-associated complications (relative risk 0.32) and a shorter duration of stay in hospital (weighted mean difference -3.24 days). ${ }^{16}$

In one trial, NIV was compared to IMV for AECOPD after a failure of standard medical treatment. There was no difference in survival. However, in those patients in whom NIV was successful, duration of hospital stay was shorter, there were fewer complications, fewer patients required de novo oxygen supplementation and there were fewer readmissions to hospital in the following year. ${ }^{158}$

No trial has demonstrated a worse outcome with NIV compared to non-ventilatory management, although, in one study, NIV use may have caused a delay in escalation to IMV. ${ }^{159}$ The danger that the use of NIV may inadvertently lead to a worse outcome is, however, suggested by a large American retrospective study. Chandra et $a l^{160}$ reported on an estimated 7.5 million admissions for AECOPD in the USA between 1998 and 2008. During this period, there was a $460 \%$ increase in the use of NIV and a $42 \%$ decline in IMV use. Worryingly, given the increasing familiarity of staff using NIV over time, the number of patients failing NIV and requiring IMV increased as did hospital mortality. By 2008, NIV failures had a 29\% risk of death, a $60 \%$ greater risk than patients managed by immediate intubation and provision of IMV. NIV failures, who were then managed by IMV, had a sevenfold greater risk of death than patients successfully treated by NIV. Possible explanations include the fact that further physiological deterioration may have resulted from the delay in the institution of IMV in NIV failures and/or that patients who fail NIV are more severely ill. ${ }^{161}$

The outcomes in AECOPD reported in the UK National COPD Resources and Outcomes project (NCROP) ${ }^{7}$ are also of concern, as NIV outcome was less favourable than reported in the RCTs discussed above. The low level of ICU involvement 
and/or use of IMV reported has led to the suggestions that the clinical environment in which NIV was delivered was inadequate for the level of patient complexity/acid-base disturbance, that there was an over-reliance on the effectiveness of NIV and an under recognition of NIV failure. ${ }^{7}$ Similar conclusions can be drawn from BTS NIV audits. In the most recent survey, carried out in 2013, median $\mathrm{pH}$ was 7.24 and yet NIV was provided outside of HDU/ICU in 91\%. In the AECOPD group (61\% of AHRF cases), overall mortality was $28 \%$ in those admitted to HDU/ICU and $40 \%$ for those admitted to admission wards. ${ }^{5}$ See Care planning and delivery of care section for further consideration of the possible unintended consequences of the introduction of NIV in managing AHRF.

In around $20 \%$ of AHRF cases secondary to AECOPD, optimised medical therapy, which includes targeting an oxygen saturation to $88-92 \%$, will result in normalisation of arterial $\mathrm{pH}^{2}{ }^{26}$ Established guidance is therefore to await improvement and initiate NIV if, after $60 \mathrm{~min}$, the following are present: $\mathrm{pH}$ $<7.35, \mathrm{pCO}_{2}>6.5 \mathrm{kPa}$ and RR $>23$ breaths $/$ min. ${ }^{148}$

There is some evidence that NIV may also be beneficial in patients with hypercapnia in the absence of acidosis. A study from China ${ }^{162}$ showed a reduction in the need for endotracheal intubation in a subgroup analysis of patients with hypercapnia but a $\mathrm{pH}>7.35$ (9/80 vs $2 / 71, \mathrm{p}=0.04)$. However, length of stay and duration of NIV were longer than in a similar UK study, ${ }^{163}$ and there was a high incidence of side effects, particularly gastric distension (23\%), despite low inflation pressure being used (IPAP 12 \pm 4 ). It is unclear if this study is applicable to UK practice.

There is insufficient evidence to support the use of absolute values of $\mathrm{pH}$ or $\mathrm{pCO}_{2}$ as an indication for IMV rather than NIV. ${ }^{164}$ Nevertheless, a pH of 7.25 has been suggested as a threshold level below which IMV should be considered. NIV may still be effective at reversing such severe acidosis but the failure rate is higher. ${ }^{158}$

\section{Evidence statements}

Optimal medical therapy, including controlled oxygen therapy, leads to a resolution of respiratory acidosis in $20 \%$ of individuals with AECOPD (Level 1+).

Compared with standard medical therapy, NIV improves survival, reduces the need for endotracheal intubation, reduces complications and reduces length of stay (Level $1+$ ).

There is no lower limit of $\mathrm{pH}$ below which a trial of NIV has been shown to be harmful (Level $2++$ ).

Continued use of NIV when the patient is deteriorating, rather than escalating to IMV, increases mortality (Level $2+$ ).

Audit data show that 'real world' outcomes do not reproduce those demonstrated in the RCTs (Level 2+).

One risk of an expansion of ward-based rather then HDU/ICU provision of NIV may be greater delay in expert review and/or escalation to IMV (Level 4).

\section{Recommendations}

24. For most patients with AECOPD, the initial management should be optimal medical therapy and targeting an oxygen saturation of $88-92 \%$ (Grade A).

25. NIV should be started when $\mathrm{pH}<7.35$ and $\mathrm{pCO}_{2}>6.5 \mathrm{kPa}$ persist or develop despite optimal medical therapy (Grade A).

26. Severe acidosis alone does not preclude a trial of NIV in an appropriate area with ready access to staff who can perform safe endotracheal intubation (Grade B).

27. The use of NIV should not delay escalation to IMV when this is more appropriate (Grade C).

28. The practice of NIV should be audited regularly to maintain standards (Grade C).

\section{Starting NIV in COPD}

Recommendations regarding investigations before starting NIV are based on expert opinion. ABG measurement is required to diagnose and quantify the severity of AHRF, and a chest radiograph is needed to seek evidence of causation or complications. To avoid any delay in giving ventilatory support, NIV should be initiated in extreme acidosis $(\mathrm{pH}<7.25)$ without waiting for a chest X-ray. Other investigations (eg, full blood count (FBC), U $+E, E C G$ ) should be performed and treatment directed at any reversible factors contributing to AHRF. In some cases, echocardiography may be indicated to exclude acute pulmonary oedema. As is further discussed in Care planning and delivery of care section, it is recommended that an action plan be agreed in the event of NIV failure and that this is documented at the start of treatment.

Good practice points

- $\mathrm{ABG}$ measurement is needed prior to and following starting NIV.

- Chest radiography is recommended but should not delay initiation of NIV in severe acidosis.

- Reversible causes for respiratory failure should be sought and treated appropriately.

- At the start of treatment, an individualised patient plan (involving the patient whenever possible) should document agreed measures to be taken in the event of NIV failure.

\section{Prognostic features relating to use of NIV in COPD}

The 2003 UK National COPD audit ${ }^{165}$ demonstrated a higher hospital mortality in patients with a lower admission $\mathrm{pH}$ and oxygen saturation, higher urea, lower albumin and older age (see below for further discussion), irrespective of treatment modality. Increased base excess (indicating chronicity of hypercapnia), MRC dyspnoea index and RR are additional prognostic variables. ${ }^{165} 166$ The presence of pulmonary consolidation on $\mathrm{X}$-ray and impaired consciousness level $(\mathrm{GCS}<8)$ increase the NIV failure rate, ${ }^{167}$ although successful outcome despite impaired consciousness has been reported. ${ }^{49} 168$

In contrast, Nava et al ${ }^{169}$ reported a good outcome for patients aged $>75$ years, in terms of intubation avoidance and reduced mortality with NIV. Others have also achieved satisfactory results in the elderly. ${ }^{170}$ However, in a retrospective analysis of 240 ward-based cases from a single centre, age $>75$ years was associated with poorer outcomes with NIV. ${ }^{171}$

The NCROP audit, which collected data on 9716 AECOPD admissions, reported mortality at $12 \%$ when the presentation $\mathrm{pH}$ was the lowest value reached, $24 \%$ when acidosis increased after presentation and 33\% when acidosis only developed after admission. ${ }^{7}$ These findings reflect a combination of increasing severity of illness and a lack of response to standard medical treatment. In addition, delay in providing therapeutic NIV and/ or IMV contributed. The audit also highlighted that a coincident metabolic acidosis was an adverse finding.

Once NIV has been initiated, a reduction in RR and improvement in $\mathrm{pH}$ within $4 \mathrm{~h}$ predicts NIV success. ${ }^{172}$ Associated features are a reduction in signs of respiratory distress, reduced anxiety or agitation and a decrease in heart rate. In one large study, Confalonieri et al ${ }^{173}$ showed that, if $\mathrm{pH}<7.25$ and $\mathrm{RR}$ $>35$ persist, NIV failure is likely. Worsening acidosis, after initial improvement with NIV, is also associated with a worse prognosis. ${ }^{174-176}$ In a case series published by Moretti et al, $20 \%$ of patients deteriorated after initially improving with NIV. In these circumstances, prognosis was poor whether patients were subsequently intubated or continued with NIV. ${ }^{177}$ 
Roche Campo et $a l^{93}$ found that polysomnographic evidence of severe sleep disturbance in patients with COPD with AHRF correlated with a poor outcome and Gursel et al, ${ }^{91}$ reporting on a retrospective analysis of patients with COPD and OHS treated in an ICU setting, found better outcome in patients receiving pressure control rather than overnight PS. Clinical research in stable sleep hypoventilation also suggests that limiting the increase in hypercapnia during sleep is important and that a controlled ventilation mode may be more advantageous than the assist mode. ${ }^{178}$

\section{Evidence statements}

Advanced age is not an important determinant of outcome with NIV treatment of AHRF (Level 1+).

An improvement in physiological parameters, usually within 1$2 \mathrm{~h}$, particularly $\mathrm{pH}$ and $\mathrm{RR}$, predict a successful outcome from NIV treatment (Level $1+$ ).

Worsening of physiological parameters, particularly $\mathrm{pH}$ and $\mathrm{RR}$, is predictive of an increased risk of death and/or requirement for intubation (Level 1+).

\section{Recommendations}

29. Advanced age alone should not preclude a trial of NIV (Grade A).

30. Worsening physiological parameters, particularly $\mathrm{pH}$ and $\mathrm{RR}$, indicate the need to change the management strategy. This includes clinical review, change of interface, adjustment of ventilator settings and considering proceeding to endotracheal intubation (Grade A).

Good practice point

If sleep-disordered breathing pre-dates AHRF, or evidence of it complicates an episode, the use of a controlled mode of NIV overnight is recommended.

\section{Duration of NIV in COPD}

Normalisation of $\mathrm{pH}$ and a $\mathrm{pCO}_{2}<6.5$ are commonly used as a guide to the discontinuation of NIV. Restoring respiratory drive will require a prolonged period of NIV to reduce the $\mathrm{pCO}_{2}$ than to correct the acidosis.

The optimal amount of NIV in the initial period, and the most effective way to withdraw it as the patient improves, have not been examined in published trials. As the work of breathing falls and acute hyperinflation reverses, as a result of treatment with steroids, antibiotics and intense broncholdilator therapy, unsupported alveolar ventilation will return towards normal. The more florid the evidence for infection precipitating AHRF, the more likely there is to be full reversal. Normalisation of $\mathrm{pCO}_{2}$ may not be possible in some patients, particularly those who show evidence of chronic hypercapnia at presentation.

In most RCTs, the intention has been that patients should receive semicontinuous NIV for the first $24 \mathrm{~h}$. The amount of NIV actually delivered, when this has been reported, has been less than planned, from a median of $20 \mathrm{~h}$ in one study ${ }^{179}$ to $7 \mathrm{~h}$ in another. ${ }^{163}$ Conventional practice is to gradually reduce the amount of time on NIV, with increasingly prolonged periods of self-ventilation during the day, while continuing with NIV overnight. Monitoring of $\mathrm{pCO}_{2}$ on and off NIV is a useful guide to how quickly the withdrawal of NIV can proceed. Transcutaneous $\mathrm{pCO}_{2}$ measurement may facilitate this better than continuing with arterial or capillary sampling. A gradual reduction of ventilator pressures, and a switch to PS or a reduction in backup rate, should mirror patient recovery. Attempts to adjust ventilator settings to achieve patient comfort remain important. Those with a less clear infective cause for AHRF, and/or evidence of chronicity of hypercapnia, should be assessed for alternative or additional causative factors such as marked fluid retention, obstructive sleep apnoea (OSA) or OHS. One study suggested there may be an advantage to employing NIV for longer than the conventional 3 days. ${ }^{162}$ More trial data are needed to guide optimal withdrawal of NIV.

\section{Evidence statement}

In clinical trials, NIV has been discontinued when there has been normalisation of $\mathrm{pH}$ and $\mathrm{pCO}_{2}$ and a general improvement in the patient's condition (Level 1+).

Recommendation

31. NIV can be discontinued when there has been normalisation of $\mathrm{pH}$ and $\mathrm{pCO}_{2}$ and a general improvement in the patient's condition (Grade B).

Good practice points

- Time on NIV should be maximised in the first $24 \mathrm{~h}$ depending on patient tolerance and/or complications.

- NIV use during the day can be tapered in the following 2 3 days, depending on pCO2 self-ventilating, before being discontinued overnight.

\section{Optimising NIV delivery and technical considerations}

The commonest reasons for failure of NIV are excessive mask leak, insufficient PS and ventilator patient asynchrony. If the PS is inadequate, alveolar ventilation will not be significantly increased. This may be detected by a lack of augmentation of chest and abdominal wall movement. National NIV audits have revealed that inadequate IPAP is often used in AECOPD. ${ }^{3}{ }^{4}$ In general, while a patient might be started on NIV with an IPAP of 15 , this should be progressively increased to reach an IPAP of 20-30 within 10-30 min, the need for higher pressure and a more rapid escalation being indicated by patient size and more severe acidosis, respectively.

In the presence of persisting hypoxaemia, that is thought unrelated to sputum retention, the EPAP may need to be increased in an attempt to recruit areas of poorly ventilated lung. (It may also be appropriate if there is a degree of upper airway obstruction). If ineffective, or if this results in distress, senior review is indicated while the $\mathrm{FiO}_{2}$ is temporarily increased.

Leak should always be minimised by mask adjustment and/or by changing the mask type. Positional upper airway obstruction may result in ineffective NIV. It is often indicated by variable mask leak. Care is needed to ensure head flexion is avoided,

Table 3 Technical issues: a guide for when NIV is failing

\begin{tabular}{|c|c|c|}
\hline Problem & Cause(s) & Solution (s) \\
\hline $\begin{array}{l}\text { Ventilator cycling } \\
\text { independently of patient } \\
\text { effort }\end{array}$ & $\begin{array}{l}\text { Inspiratory trigger } \\
\text { sensitivity is too } \\
\text { high } \\
\text { Excessive mask leak }\end{array}$ & $\begin{array}{l}\text { Adjust trigger } \\
\text { Reduce mask leak }\end{array}$ \\
\hline $\begin{array}{l}\text { Ventilator not triggering } \\
\text { despite visible patient effort }\end{array}$ & $\begin{array}{l}\text { Excessive mask leak } \\
\text { Inspiratory trigger } \\
\text { sensitivity too low }\end{array}$ & $\begin{array}{l}\text { Reduce mask leak } \\
\text { Adjust trigger } \\
\text { For NM patients consider } \\
\text { switch to PCV }\end{array}$ \\
\hline $\begin{array}{l}\text { Inadequate chest expansion } \\
\text { despite apparent triggering }\end{array}$ & $\begin{array}{l}\text { Inadequate Tidal } \\
\text { volume }\end{array}$ & $\begin{array}{l}\text { Increase IPAP. In NM or } \\
\text { chest wall disease } \\
\text { consider longer Ti }\end{array}$ \\
\hline Chest/abdominal paradox & $\begin{array}{l}\text { Upper airway } \\
\text { obstruction }\end{array}$ & $\begin{array}{l}\text { Avoid neck flexion } \\
\text { Increase EPAP }\end{array}$ \\
\hline $\begin{array}{l}\text { Premature expiratory effort } \\
\text { by patient }\end{array}$ & Excessive Ti or IPAP & Adjust as necessary \\
\hline
\end{tabular}

Davidson AC, et al. Thorax 2016;71:ii1-ii35. doi:10.1136/thoraxjnl-2015-208209 
particularly in sleep. Patient-ventilator asynchrony may be caused by mask leak, insufficient or excessive IPAP, inappropriate setting of Ti or Te, high levels of intrinsic PEEP or excessively sensitive triggers. If the cause is unclear, advice should be sought from an experienced NIV practitioner.

Although there is no agreed definition of NIV failure, it is suggested by persisting or worsening of acidosis despite attempts to optimise NIV delivery. In these circumstances, further advice should be sought as soon as possible. NIV failure is associated with low/falling $\mathrm{pH}^{172}$ and a high APACHE II score. ${ }^{180}$ Persisting with ineffective NIV adds to patient discomfort and, if IMV is indicated, risks further patient deterioration and cardiorespiratory arrest. Evidence that this risk is real comes from the use of NIV in post-extubation respiratory failure where delay in re-intubation, caused by persisting with NIV when ineffective, increased mortality. ${ }^{181}$ If NIV is adding to patient distress, and intubation has been deemed to be inappropriate (see below), NIV should be discontinued and palliative care measures adopted.

Good practice point

Before considering NIV to have failed, check that common technical issues have been addressed and ventilator settings are optimal.

\section{Indications for IMV in AECOPD}

Intubation should be immediately considered for patients presenting with or developing respiratory arrest, gasping respiration, a $\mathrm{pH}<7.15$ or showing signs of a low cardiac output. Intubation may also be appropriate if NIV is contra-indicated or technically impossible and when NIV has been tried but has failed.

There is insufficient evidence to support the use of absolute values of $\mathrm{pH}$ or $\mathrm{pCO}_{2}$ as intubation criteria and it is unlikely that any absolute value would be applicable to all patients in all situations. ${ }^{164}$ Nevertheless, $\mathrm{pH}<7.25$ has been suggested as a level below which IMV should be considered and $<7.15$ as the level that IMV is indicated (following initial resuscitation and use of controlled oxygen).

In the UK, only a small proportion of patients receiving NIV treatment escalate to IMV despite data suggesting more should do so. ${ }^{3} 47$ A degree of unjustified 'therapeutic nihilism' may have shaped UK IMV practice. Duration of ICU stay and survival in AECOPD is better than most other medical causes for which invasive ventilation is employed. ${ }^{6}$ In a prospective cohort study, clinicians' estimated prognosis for patients with AECOPD or chronic asthma was lower than indicated by predictive modelling. ${ }^{182}$

Specialist support to staff providing NIV may reduce mortality. In one study, employing critical care outreach nurses, the mortality was reduced from $57 \%$ to $35 \%$. This was in part due to a greater number of patients receiving IMV. ${ }^{183}$ Validated prognostic scoring tools (see next section) may aid discussion regarding intubation. Box 1 summarises the indications for IMV in AECOPD.

\section{Evidence statements}

Intubation is indicated if NIV is failing (unless it is agreed that this is not desired by the patient or it is deemed not in the patient's 'best interest') (Level 1+).

Neither patient characteristics nor pathophysiological parameters are sufficiently robust to predict the success of NIV or IMV but, in general, the more adverse features that are present and the greater the physiological disturbance the higher the chance of treatment failure or death (Level $2++$ ).

\section{Recommendations}

32. IMV should be considered if there is persistent or deteriorating acidosis despite attempts to optimise delivery of NIV (Grade A).
Box 1 Indications for invasive mechanical ventilation (IMV) in acute exacerbation of COPD (AECOPD)

- Imminent respiratory arrest

- Severe respiratory distress

- Failure of or contra-indications to non-invasive ventilation (NIV)

- Persisting $\mathrm{pH}<7.15$ or deterioration in $\mathrm{pH}$ despite NIV

- Depressed consciousness (Glasgow Coma Score <8)

33. Intubation should be performed in respiratory arrest or periarrest unless there is rapid recovery from manual ventilation/ provision of NIV (Grade D).

34. Intubation is indicated in management of AHRF when it is impossible to fit/use a non-invasive interface, for example, severe facial deformity, fixed upper airway obstruction, facial burns (Grade D).

35. Intubation is indicated where risk/benefit analysis by an experienced clinician favours a better outcome with IMV than with NIV (Grade D).

\section{Outcome following NIV or IMV in AECOPD}

There are a number of tools that may inform discussion regarding prognosis in COPD. Some were developed for use in the stable setting, such as the BODE index ${ }^{184}$ and the DECAF score. ${ }^{185}$ APACHE II, a generic acute physiology score, was developed using parameters available at ICU admission. Despite being generic, it retains predictive value of mortality in AECOPD. ${ }^{180} 186187$ Wildman et $a l^{188}$ analysed a large UK ICU clinical database (with a $35 \%$ mortality) to develop a diseasespecific score, the COPD and Asthma Physiology Score (CAPS). This was based on 8 , mainly biochemical, variables. CAPS was reported to perform better than generic scoring. The authors acknowledge that normal functional assessment, for example, by body mass index (BMI), usual functional status and presence of comorbidity, might improve predictive power.

Confalonieri et al $^{189}$ suggested that prognosis following successful use of NIV in AHRF was better than if IMV were employed. The number and length of further hospitalisations were significantly higher and the survival rate at 12 months significantly lower $(50 \%$ vs $71 \%)$ than in patients who received NIV. Follow-up of patients in the RCT of Plant et al ${ }^{172}$ showed a median survival of 16.8 months in those treated with NIV and 13.4 months in those receiving standard treatment $(p=0.12)$. The trend in improved survival was attributable to prevention of death during the index admission.

A study of an inception cohort of 73106 patients with COPD, followed up after their first AHRF treated by NIV, reported a 2-year survival of $70 \%$ and a median survival of 3.6 years. ${ }^{190}$ After a second hospitalisation, patients typically entered a deteriorating pattern with more frequent and severe episodes until death. A retrospective analysis of 100 patients with COPD, followed for up to 5 years after their first episode of NIV, ${ }^{191}$ found that $52 \%$ survived 2 years. When the BMI was $<22 \mathrm{~kg} / \mathrm{m}^{2}$, age $>75$ years or there was prior home oxygen use, survival was only $26 \%$. In a prospective cohort of patients with COPD surviving AHRF treated by NIV, ${ }^{192} 80 \%$ were re-admitted within a year, of whom 50\% died. APACHE II score at admission, home oxygen prescription and a BMI below 25 predicted early recurrent AHRF or death. 
In summary, an admission with AHRF is a critical point in the natural history of COPD, with a more accelerated decline in most patients following recovery. It indicates a high risk of recurrence of AHRF and poor long-term prognosis. Survival data vary between published studies, presumably a reflection of differences in case mix. Survival might be better if NIV is successfully employed for AHRF than if IMV is used. An important management point is that the first episode of AHRF should prompt a discussion about lifestyle, patient wishes for management of future episodes and discussions about end-of-life care generally.

There is evidence of 'prognostic pessimism' among clinicians caring for patients with AECOPD. In an outcome study of 517 patients, $62 \%$ survived to 180 days, yet overall predicted survival at the time of admission was $49 \% .{ }^{182}$ For those considered to be in the worst prognostic group (a survival rate of 10\%), 40\% recovered. Accordingly, as survival from AECOPD becomes less likely, clinicians become worse at prediction and err on the side of underestimating survival. By implication, it is likely that patients who might otherwise survive are currently being denied admission to ICU because their survival potential is underestimated. Importantly, from a patient perspective, Wildman et al ${ }^{193}$ reported that the majority of patients surviving IMV for AHRF had stable and acceptable QoL despite poor health status and 96\% stated they would opt for IMV again under similar circumstances.

Evidence statement

There are validated tools for the assessment of prognosis in stable and exacerbating COPD populations but, on their own, they are unreliable for individual prognostication (Level $2++$ ).

Physicians underestimate survival potential in AECOPD treated by IMV (Level $2+$ ).

The majority of patients with COPD or chronic asthma who receive IMV would elect to receive it again (Level $2+$ ).

An episode requiring ventilatory support generally indicates advanced disease with a high risk for future episodes of AHRF and a limited prognosis (Level $2++$ ).

\section{Recommendations}

36. Prognostic tools may be helpful to inform discussion regarding prognosis and the appropriateness of IMV with the caveat that such tools are poorly predictive for individual patient use (Grade B).

37. Clinicians should be aware that they are likely to underestimate survival in AECOPD treated by IMV (Grade B).

38. Clinicians should discuss management of possible future episodes of AHRF with patients following an epsiode requiring ventilatory support because there is a high risk of recurrence (Grade B).

\section{Acute asthma}

Five small RCTs ${ }^{194-198}$ of NIV in acute asthma have been published. Four were conducted in the ED and one in a respiratory ICU. Importantly, none of the RCTs included patients with hypercapnia and intubation rates were low. ${ }^{199}$ Most showed treatment with NIV led to a faster improvement in FEV1 and a shorter ICU/hospital stay. They all have important design weaknesses. The trial by Soma et al ${ }^{197}$ lacked a second control arm (conventional inhaled bronchodilators) and the trial by Brandao et $a l^{194}$ did not give systemic steroids. No information was provided about acceptability of NIV to patients. The only study reporting use in AHRF asthma was a retrospective cohort study by Meduri et $a l^{200}$ of 17 patients with a mean $\mathrm{pH}$ of 7.25. NIV was reported to be successful in avoiding intubation in 15 .

The use of NIV in acute asthma, particularly AHRF, needs to be set in the context of a very low mortality rate with IMV. ${ }^{201}$ There is also the potential for patients with acute asthma to deteriorate rapidly, to require high inflation pressures and a high inspired oxygen concentration. Trialling NIV therefore carries significant risk. The patient with brittle asthma or a very short history suggesting hyperacute brochospasm, especially when oxygen toxicity in transit is implicated, might justify a trial of NIV in the resucitation area but, in all other circumstances, ventilatory support should be by intubation and provision of IMV. The overall invasive management of acute severe asthma is similar to that in AECOPD but a higher $\mathrm{SaO}_{2}$ target of $96 \%$ is advised. For more specialist consideration, the reader is referred to standard textbooks or recent reviews.

Acute (and chronic) hypercapnia may complicate chronic asthma, a condition that shares many features of COPD, such as chronic hyperinflation, persistent and only partially reversible airflow obstruction, mucus hypersecretion and infective exacerbations. As the pathophysiology is similar, the guidance on the use of NIV and IMV in AECOPD applies to the chronic asthmatic with AHRF.

\section{Evidence statements}

There is insufficient evidence to support the use of NIV in AHRF in acute asthma (Level 3).

IMV in acute asthma carries a very low mortality rate. Most asthma deaths relate to presentation in extremis or a failure to immediately implement IMV when indicated rather than a failure of IMV per se (Level 2+).

Recommendations

39. NIV should not be used in patients with acute asthma exacerbations and AHRF (Grade C).

40. Acute (or acute on chronic) episodes of hypercapnia may complicate chronic asthma. This condition closely resembles COPD and should be managed as such (Grade D).

\section{Non-CF bronchiectasis}

Recurrent episodes of hypercapnic respiratory failure may characterise bronchiectasis with periods of good or acceptable quality of life (QoL)/health status in the intervening months or years. In some, domiciliary ventilation will be indicated for symptoms of sleep disordered breathing. There are no RCTs of NIV versus IMV in acute exacerbations of bronchiectasis. The recommendations regarding NIV for AECOPD are appropriate although there is the additional challenge of excessive and difficult to clear sputum. NIV may relieve breathlessness and help patients to participate more effectively with physiotherapy. A mini-tracheostomy, or other techniques to aid sputum clearance, may be indicated. ${ }^{202}$

There is scant data on outcomes for AHRF in non-CF bronchiectasis. In a retrospective review ${ }^{203}$ of patients managed by NIV $(n=31)$ or IMV $(n=26)$ for AHRF, the NIV group had less severe physiological disturbance. There was no difference in hospital mortality between the two groups (26\% and $27 \%$ ). The NIV failure rate (need for intubation, or death in the ICU) was $33 \%$. Using logistic regression, the APACHE II score was the only predictor of hospital mortality (OR 1.19 per point) and the severity of hypoxia $\left(\mathrm{pO}_{2} / \mathrm{FiO}_{2}\right.$ ratio) the only predictor of NIV failure (OR $1.02 / \mathrm{mm} \mathrm{Hg}$ decrease). Hospital mortality of patients with AHRF secondary to bronchiectasis is therefore approximately $25 \%$ whether management is by NIV or IMV. When selectively applied, NIV fails in one-third and this is predicted by the degree of hypoxaemia. Similar criteria should be used as in AECOPD when deciding appropriateness of intubation: health status, comorbidities, previous episodes of IMV and patient preferences. Evidence of an acute precipitating factor (infection) should favour intubation, as reversibility is more likely than in progressive chronic hypercapnia. 


\section{Evidence statements}

In non-CF bronchiectasis and AHRF, NIV is indicated if there is respiratory acidosis using the same criteria as in AECOPD (Level 3).

Outcome with NIV is no worse than with IMV in selected patients (Level 2+).

\section{Recommendations}

41. In patients with non-CF bronchiectasis and AHRF, controlled oxygen therapy should be used (Grade D).

42. In patients with non-CF bronchiectasis, NIV should be started in AHRF using the same criteria as in AECOPD (Grade B).

43. In patients with non-CF bronchiectasis, NIV should usually be tried before resorting to IMV in those with less severe physiological disturbance (Grade C).

44. In non-CF bronchiectasis, the patient's clinical condition prior to the episode of AHRF, and the reason for the acute deterioration, should be evaluated and used to inform the decision about providing IMV (Grade C).

Good practice points

- In patients with non-CF bronchiectasis, the precipitating cause is important in determining short-term prognosis.

- Health status prior to the episode of AHRF is an important predictor of outcome.

\section{Cystic fibrosis}

Recurrent episodes of acute on chronic hypercapnic respiratory failure characterise advanced CF, such episodes usually being preciptated by infection. There may be intervening months of acceptable QoL/health status. There are no RCTs of NIV versus IMV in AHRF and the recommendations regarding NIV for AECOPD remain appropriate. Hypoxaemia is often more severe than in AECOPD, in some, relating to co-existent pulmonary hypertension. Secretion clearance is also a major issue and may render NIV ineffective or poorly tolerated. ${ }^{204-207}$ Case series of patients with CF receiving NIV as a bridge to transplantation have been reported. 208209

As the outcome of invasive ventilation in CF is generally poor, it has been recommended that NIV be used preferentially. ${ }^{202}$ In a retrospective multicentre study of 60 ICU hospitalisations for 42 adult patients with CF admitted between 2000 and 2003, NIV was used in $57 \%$ and was successful in $67 \%$ of these. ${ }^{210}$ Endotracheal intubation was implemented on 19 occasions and ICU mortality was $14 \%$. Among recognised markers of CF disease severity, only the annual FEV1 loss significantly related to outcome $(\mathrm{HR}=1.47, \mathrm{p}=0.001)$. Admission SAPS II, a pathophysiological score, weakly predicted outcome $(\mathrm{HR}=1.08$, $\mathrm{p}<0.001$ ), but the perceived need for endotracheal intubation strongly predicted mortality $(\mathrm{HR}=16.60, \mathrm{p}<0.001)$. In a study from a single centre, 30 patients were managed by IMV on 34 occasions. $^{211}$ Eleven patients died in the ICU and a further seven before hospital discharge. Sixty per cent intubated for pneumothorax and/or haemoptysis survived contrasting, with only $30 \%$ when intubated for infection. Mean survival postdischarge was 447 days. There were no significant differences in survivors for colonising organism, frequency of infective exacerbations or acute severity of illness. A greater fall in BMI over the preceding 24 months was more frequent in non-survivors. The authors concluded that patients with CF developing AHRF due to haemoptysis and/or pneumothorax should be considered for management by IMV.

Evidence statements

Chronic disease markers are more relevant than rates of hospitalisation or FEV1 decline in assessing outcome in AHRF complicating CF (Level 2+).
When ventilatory support is needed, outcome following IMV is worse than with NIV, especially when infection is the precipitant (Level 2+).

Secretion clearance is a major issue and may render NIV ineffective or poorly tolerated (Level 2-).

\section{Recommendations}

45. In patients with CF, controlled oxygen therapy should be used in AHRF (Grade D).

46. In patients with CF, NIV is the treatment of choice when ventilatory support is needed (Grade C).

47. In patients with CF, specialised physiotherapy is needed to aid sputum clearance (Grade D).

48. In patients with $\mathrm{CF}$, a mini-tracheostomy combined with NIV may offer greater chance of survival than resorting to IMV (Grade D).

\section{Restrictive lung disease}

The causes of AHRF include severe chest wall deformity, neuromuscular conditions that affect the respiratory muscles and OHS. Presentation is often with advanced chronic hypercapnia. An insidious decline in health may not have been medically recognised as being due to the development of respiratory failure. Acute presentations, often with infection precipitating acute illness, are likely when the VC is $<1 \mathrm{~L}$. Unlike AECOPD, recurrent critical episodes do not preclude intervening good life quality, acceptable health status and prolonged survival. There are no RCTs to guide practice in AHRF and the recommendations presented are extrapolated from the AECOPD literature, from reports of the value of domiciliary NIV (most evidence coming from trials in the more progressive NMDs) and from expert opinion.

\section{NMD and CWD}

Respiratory impairment generally parallels disease progression in NMD. However, in some, diaphragm involvement precedes locomotor disability and presentation with acute on chronic hypercapnia is typical. This pattern is characteristic of acid maltase deficiency and the amyotrophic lateral sclerosis variety of motor neurone disease. In some of the muscular dystrophies, bulbar muscle involvement is common. As a result, sleep disordered breathing may arise from a combination of respiratory muscle weakness and upper airway obstruction. The resulting nocturnal hypoventilation will then gradually spill over into daytime hypercapnia. Bulbar dysfunction also renders voluntary cough less effective. NICE had previously published guidance on the use of NIV in motor neurone disease, ${ }^{212}$ which did not consider management of acute illness nor the value of intubation if NIV fails. New guidance from NICE on motor neurone disease is in preparation. While respiratory failure is predictable in the majority, some MND patients present before a formal diagnosis has been made. ${ }^{213} 214$ This also occurs in less progressive conditions such as Limb Girdle muscular dystrophy or Myotonic Dystrophy. Without domicilary NIV, the natural history of neuromuscular and CWDs is of progressive chronic hypercapnic failure leading to death. It is well recognised such individuals can survive long term on home NIV with a good QoL, even if they present initially in severe respiratory failure. Thus individuals with NMD and CWD who present with AHRF should not be denied acute NIV. The success of domicilary NIV has made the management of any associated cardiomyopathy increasingly clinically relevant.

In CWD, evidence of pre-existing sleep disordered breathing is also common at AHRF presentation. In some, marked chronic hypercapnia is an unexpected finding when ABGs are 
performed. Such patients may have established pulmonary hypertension, chronic hypoxaemia and polycythaemia.

In contrast to AECOPD, where the degree of acidosis is more important than the degree of hypercapnia, any elevation of $\mathrm{pCO}_{2}$ in $\mathrm{NMD} / \mathrm{CWD}$ may herald an impending crisis. ${ }^{213}$ Patients have a reduced respiratory reserve but may initially sustain sufficient alveolar ventilation to maintain normal carbon dioxide tension. Minor infection, such as coryza, may be provocative and over the next 24-72 h progressive hypercapnia may develop. Tolerance of acute and chronic hypercapnia varies considerably. Some patients are excessively sleepy with minimal elevation of $\mathrm{pCO}_{2}$, while others remain alert despite much more severe hypercapnia. NIV should be considered in any breathless/acutely unwell patient with NMD/CWD before respiratory acidosis develops.

In the absence of bulbar dysfunction, NIV is usually well tolerated in the restrictive causes of AHRF. Unless there is significant skeletal deformity, a low degree of PS (eg, a pressure difference of $8-12 \mathrm{~cm}$ ) is needed in NMD. By contrast, in severe kyphoscoliosis, an IPAP $>20$, and sometimes up to 30 , may be required because of the high impedance to inflation. Expiratory flow is normally not limited in either restrictive category and the inspiratory/expiratory time (IE) ratio for the backup rate (or PCV) should initially be set at 1:1 to allow an adequate time for inspiration. Bulbar dysfunction renders effective NIV more difficult to achieve, requires a higher EPAP to overcome upper airway obstruction and needs special attention to aid cough and the clearing of upper and lower airways. Clinical experience in providing NIV is needed to best titrate the EPAP. A modest increase in the domiciliary ventilator settings is advised in the case of home mechanical ventilation patients being admitted with AHRF.

While triggering is usually normal in CWD, it is commonly inadequate in the other restrictive conditions. Many patients with NMD find PCV more comfortable and this may also more effectively control nocturnal hypoventilation.

Evidence statement

There are no trials comparing NIV with IMV in AHRF in NMD or CWD. Domiciliary NIV is effective in treating chronic hypercapnia, improves long-term survival and preserves a good or acceptable QoL (Level 4).

Recommendations

49. Controlled oxygen therapy should be used in patients with NMD or CWD and AHRF (Grade D).

50. NIV should almost always be trialled in the acutely unwell patient with NMD or CWD with hypercapnia. Do not wait for acidosis to develop (Grade D).

51. In patients with NMD or CWD, NIV should be considered in acute illness when $\mathrm{VC}$ is known to be $<1 \mathrm{~L}$ and $\mathrm{RR}>20$, even if normocapnic (Grade D).

52. In patients with NMD or CWD, consider controlled ventilation as triggering may be ineffective (Grade D).

53. In NMD and CWD, unless escalation to IMV is not desired by the patient or is deemed to be inappropriate, intubation should not be delayed if NIV is failing (Grade D).

Good practice points

- Individuals with NMD and CWD who present with AHRF should not be denied acute NIV.

- NIV is the ventilation mode of choice because patients with NMD or CWD tolerate it well and because extubation from IMV may be difficult.

- In patients with NMD or CWD, deterioration may be rapid or sudden, making HDU/ICU placement for therapy more appropriate.
- In patients with NMD or CWD, senior/experienced input is needed in care planning and is essential if differences in opinion exist or develop between medical staff and patient representatives.

- In patients with NMD, it should be anticipated that bulbar dysfunction and communication difficulties, if present, will make NIV delivery difficult and may make it impossible.

- Discussion about NIV and IMV, and patients' wishes with respect to cardiopulmonary resuscitation, should occur as part of routine care in patients with NMD or CWD.

- In patients with NMD or CWD, nocturnal NIV should usually be continued following an episode of AHRF pending discussion with a home ventilation service.

NIV failure and discontinuing NIV following recovery in NMD and CWD Decisions regarding resuscitation and intubation can be particularly challenging as little or no evidence exists for most of the causative conditions, communication with the patient may be difficult and/or cognition be impaired and there may be unreasonable expectation on the part of families and carers. A resuscitation plan is important but may be difficult to negotiate. Inability to clear secretions is a common cause of NIV failure. This may result from an excessive volume of secretions or from a combination of limited inspiratory capacity, expiratory muscle weakness and bulbar dysfunction. Specialist advice and experience is required to manage NIV in the presence of bulbar dysfunction and to provide effective cough assistance. ${ }^{215}$ As with all patients, good communication is important. As this may be a challenge, it is another reason for seeking specialist help and advice. Enlisting the help of normal carers may be useful because they may engender more reassurance to patients and be better at aiding sputum clearance.

Recovery usually takes longer than in AECOPD, so that stepping down the time on NIV should proceed more slowly, and NIV will need to be continued overnight. The higher the presentation $\mathrm{HCO}_{3}$, the longer the period of relative hyperventilation required to reduce buffering capacity. A target $\mathrm{pCO}_{2}$ around $6.5 \mathrm{kPa}$ self ventilating is recommended. Following recovery, the majority of individuals with NMD or CWD will require NIV at home. NIV should continue overnight until discussion with a home ventilation service.

Good practice points

- In patients with NMD or CWD, intolerance of the mask and severe dyspnoea are less likely to cause NIV failure. Bulbar dysfunction makes NIV failure more likely.

- Deterioration in patients with NMD or CWD may be very sudden. Difficulty achieving adequate oxygenation or rapid desaturation during a break from NIV are important warning signs.

- In patients with NMD or CWD, the presence of bulbar dysfunction, more profound hypoxaemia or rapid desaturation during NIV breaks suggests that placement in HDU/ICU is indicated.

\section{IMV in NMD/CWD}

Many clinicians have limited experience of managing NMD and CWD. There is the danger of underestimating survival potential in the face of severe general disability. Patient choice and seeking the views of advocates when communication with the patient is difficult are paramount. Discussion with a specialist centre on both the delivery of IMV and weaning is recommended.

The risk of sudden deterioration is greater due to reduced respiratory reserve, impaired cough, cardiomyopathy (possibly undiagnosed) and sometimes communication challenges. Intubation practice, elective or in AHRF, varies between centres 
and between countries. For instance, in motor neurone disease (MND), elective intubation is reported to occur in $0.8 \%$ (Ireland), 6\% (USA) and 10.6\% (Italy) of cases. ${ }^{214}$

Outcome data following IMV are limited to case series in MND and OHS. These reports usefully illustrate shared issues in progressive NMD and many patients with advanced CWD. One report of outcome in MND following intubation for AHRF highlighted that $50 \%$ of patients were undiagnosed at the time of intubation, only $17 \%$ weaned and few left hospital. ${ }^{216}$ Recently, Sancho $e t a l^{217}$ reported a median survival of 1 year in patients intubated after failing acute NIV. Chio et $a l^{214}$ reported on $1260 \mathrm{MND}$ cases, over an 8-year period, from a single Italian neurology centre; 134 patients received IMV, which was initiated as an emergency in $40 \%$. Median survival was 250 days. Death occurred in hospital in $20 \%$, at home in $48 \%$ and in a nursing home in $32 \%$. Neither patient experience nor economic analysis was reported.

The outcome of patients with MND referred to a specialist weaning service in the UK was examined by Chadwick et al. ${ }^{218}$ Thirty patients had been transferred over a 15 -year period. Diagnosis followed intubation in 17. In 14 patients, extubation to long-term NIV was possible, of whom 9 were non-bulbar cases and 10 returned home. Thirteen remained tracheostomy ventilated, of whom 9 were bulbar and 7 returned home. Median survival from tracheal intubation was 13.7 months $(95 \%$ CI 0 to 30.8) for those known to have MND and 7.2 months (95\% CI 5.1 to 9.4) for those not previously diagnosed.

There has been a call for the value of IMV in MND to be re-evaluated both as an elective policy and at the time of crisis. ${ }^{219}$ In many of the other NMDs, for example, acid maltase deficiency and Duchene Muscular Dystrophy, a more prolonged survival rate with a good QoL is to be expected following recovery from AHRF, and an aggressive approach to managing it is, in the opinion of the guideline group, more justified than has historically been the case in the UK. It is also what most patients and their families want. Expert experience is that the majority of patients will survive a period of IMV. Comorbidity, especially associated cardiomyopathy, is important prognostically. The weaning process is often prolonged but, in the absence of severe bulbar dysfuntion, many can be safely extubated onto NIV and avoid a tracheostomy. Should this fail, and a tracheostomy be required, specialist centre experience is that subsequent decannulation is possible in most. While long-term survival may be limited, QoL may be acceptable and health status may improve with domicilary NIV. This is particularly the case in the more slowly progressive NM conditions and in stable CWD. In the latter group, even advanced pulmonary hypertension may resolve.

Evidence statements

There are national (and centre) differences in use of IMV in AHRF complicating motor neurone disease (Level 3).

The diagnosis of motor neurone disease, and other neuromuscular conditions, is sometimes only made after admission to the ICU for IMV (Level 3).

De-cannulation of a tracheostomy is more difficult when there is bulbar disease (Level 3).

Planned elective domiciliary NIV is preferable to crisis management in NMD and CWD. This reduces the risk of acute presentation and provides a proven alternative to IMV which risks prolonged or permanent tracheostomy ventilation (Level 3). Recommendations

54. In patients with NMD or CWD, senior staff should be involved in decision-making, in conjunction with home mechanical ventilation specialists, if experience is limited, and especially when the appropriateness of IMV is questioned (Grade D).
55. Advance care planning, particularly around the potential future use of IMV, is recommended in patients with progressive NMD or CWD. This may best be supported by elective referral to a home ventilation service (Grade D).

\section{IMV strategy in NMD and CWD}

In patients with NMD without significant chest wall distortion, the impedance to inflation is low. It is rarely necessary to use an IPAP above 20. It should initially be set at 10 and increased according to the resulting tidal volume. In contrast, patients with kyphoscoliosis usually require high inflation pressures. Expiration is generally not flow limited but impedence is typically high so that an I:E ratio of 1 to 1 is recommended in both diagnostic groups.

When lung volume is reduced, there is radiological evidence of lobar collapse or unexplained hypoxia, the PEEP setting on the ventilator may need to be increased up to or above $10 \mathrm{~cm} .{ }^{14}{ }^{15}$ Adjustments should be individualised according to ventilatory parameters ( $R R$, dynamic compliance, plateau pressure) and patient comfort.

Good practice points

- Patients with NMD usually require low levels of PS.

- Patients with chest wall deformity usually require higher levels of PS.

- PEEP in the range $5-10$ is commonly required to increase residual volume and reduce oxygen dependency in both patient groups.

\section{Obesity hypoventilation syndrome}

In obese patients, hospitalised for any reason, the presence of hypercapnia increases morbidity and mortality. ${ }^{220}$ Despite this, currently, there is a lack of evidence to guide treatment of either chronic hypercapnia or AHRF complicating obesity. One nonrandomised trial suggested that long-term survival is better in those who accept treatment for sleep disordered breathing compared with those who do not. ${ }^{221}$ Severe OSA is the principal cause of hypercapnia, but hypoventilation also results from the mechanical effect of obesity. 22

Presentation with acute on chronic respiratory failure is more common than de novo AHRF but the precipitant cause for destabilisation may be unclear. Not uncommonly, chronic hypercapnia is unexpectedly revealed peri-operatively following routine or emergency surgery in an obese patient not known to have OHS. The possibility of OSA/OHS in the morbidly obese $(\mathrm{BMI}>35)$ needs to be borne in mind by surgical and anaesthetic teams.

In the absence of evidence, we recommend that the indications for NIV in the breathless obese patient should be the same as in AECOPD ie $\mathrm{pCO}_{2}>6.5$ and $\mathrm{pH}<7.35$. Additionally, NIV should be considered in any patient admitted to hospital with a raised $\mathrm{pCO}_{2}$ who is excessively somnolent or when there is evidence of fluid retention. Following recovery, patients will need to be referred to an HMV centre. Patients with OHS can sometimes be switched to CPAP at a later date.

\section{Evidence statements}

In patients with OHS, NIV is indicated if there is respiratory acidosis using the same criteria as in AECOPD (Level 1-).

In the absence of acidosis, NIV may be indicated in some hypercapnic and/or somnolent obese patients (Level 2+).

\section{Recommendations}

56. Controlled oxygen therapy should be used in patients with OHS and AHRF (Grade D).

57. In patients with OHS, NIV should be started in AHRF, using the same criteria as in AECOPD (Grade B). 
58. NIV is indicated in some hospitalised obese hypercapnic patients with daytime somnolence, sleep disordered breathing and/or right heart failure in the absence of acidosis (Grade D).

\section{NIV settings and placement in OHS}

Obese patients with severe AHRF have a significant risk, despite receiving NIV, of sudden deterioration and are likely to be difficult to intubate (see below). Upper airway obstruction is common and will be more apparent during sleep. It may persist, despite increasing the EPAP, as indicated by intermittent abdominothoracic paradox during NIV 'assisted' breaths. Another clue is intermittent mask leak that accompanies obstructed inspiration. A more upright position may help, but an EPAP in the 10-15 range is often required. Expert assessment is recommended to titrate the EPAP. Tidal volume may be compromised by high level EPAP and, in some, the impedance to inflation is very high and an IPAP of $>30$ is required. ${ }^{217}$ Prolonging Ti will increase the resulting Vt delivered so a I:E ratio of $1: 1$ is advised. If the resulting $\mathrm{Vt}$ is still inadequate, consideration should be given to using volumecontrolled ventilation or a volume-assured mode, ${ }^{92}$ although the benefits of the latter are currently unproven. Different EPAP settings may be appropriate depending on sleep/awake state.

Good practice points

- High IPAP and EPAP settings are commonly required in patients with OHS (eg, IPAP >30, EPAP >8).

- Volume control (or volume assured) modes of providing NIV may be more effective when high inflation pressures are required.

\section{NIV failure in $\mathrm{OHS}$}

In patients with OHS, the same indicators suggest a failing patient and the same troubleshooting solutions apply as in AECOPD (see table 3). Fluid retention is common and its extent is commonly under-estimated. It may be in excess of $20 \mathrm{~L}$. Achieving an $\mathrm{SaO}_{2} 88-92 \%$ may be difficult and relates to collapse of dependent lung and/or reflects underlying pulmonary vascular disease. Sudden and precipitous falls in oxygenation may follow temporary removal of NIV. If high EPAP settings fail to improve the A-a gradient, a ventilator offering oxygen blending may be required. Difficulty in clearing secretions may contribute to poor gas exchange.

Good practice points

- Fluid overload commonly contributes to ventilatory failure in patients with OHS and its degree is easily underestimated.

- Forced diuresis may be useful.

- As the risk of NIV failure is greater, and intubation may be more difficult, placement in HDU/ICU for NIV is recommended.

\section{Discontinuing NIV in OHS}

During wakefulness, weaning of NIV should proceed as in AECOPD. NIV overnight should be continued pending discussion with the local home ventilation service. Other aspects, such as consideration of bariatric surgery and optimal EPAP settings when returning home, are important aspects of continuing care. Good practice points

- NIV can be discontinued as in patients with AECOPD.

- Many patients with AHRF secondary to OHS will require long-term domiciliary support (CPAP or NIV).

- Following an episode of AHRF, referral to a home ventilation service is recommended.
IMV strategy in OHS

Intubation can be challenging and patient deterioration may be rapid. There is also a higher risk of aspiration. Pressure control is recommended until stability has been achieved and should be initially set at 20 and increased according to the resulting tidal volume. Inspiratory pressure in excess of 30 may be required. To recruit collapsed lung, PEEP may need to be $10-15 \mathrm{~cm} .{ }^{14} 15$ It should be adjusted according to ventilatory parameters (RR, dynamic compliance, plateau pressure) and patient comfort.

Good practice points

- In patients with OHS, pressure-controlled MV is recommended initially.

- In patients with OHS, high PEEP settings may be needed to recruit collapsed lung units and correct hypoxaemia.

- In patients with OHS, forced diuresis is often indicated.

\section{WEANING FROM IMV \\ Introduction}

Weaning is defined as the progressive reduction of ventilatory support leading up to extubation. Delayed weaning complicates $6 \%$ of patients managed by IMV but consumes $37 \%$ of ICU resources. ${ }^{223}$ In one study, up to $50 \%$ of patients who selfextubated did not require re-intubation, ${ }^{224}$ implying that many patients are treated with IMV for longer than necessary. Clinical criteria to be met before starting weaning are detailed below: 225226

- Adequate oxygenation: $\mathrm{PaO}_{2} / \mathrm{FiO}_{2}$ ratio $>27 \mathrm{kPa}(200 \mathrm{~mm} \mathrm{Hg})$

- $\mathrm{FiO}_{2}<0.5$

- PEEP $<10 \mathrm{~cm} \mathrm{H}_{2} \mathrm{O}$

- Adequate alveolar ventilation $\left(\mathrm{pH}>7.3, \mathrm{pCO}_{2}<6.5 \mathrm{kPa}\right)$.

Fluid balance should also be optimised. The detrimental effect of excess hydration is now recognised in sepsis ${ }^{227}$ and in acute lung ${ }^{228}$ and kidney injury. ${ }^{229}$ A positive fluid balance adversely affects alveolar ventilation, oxygenation, weaning progress and extubation outcome. ${ }^{224} 230$ Brain Natriuretic Peptide (BNP) has been reported to predict failure to wean and correlates with weaning duration; a BNP-directed fluid management strategy has been reported to shorten time to extubation, particularly in patients with left ventricular dysfunction. ${ }^{231}$

Evidence statements

Easily measured clinical parameters indicate when weaning can start (Level 2+).

Excess fluid administration may delay weaning or contribute to its failure (Level 2++).

In left ventricular dysfunction, a BNP-directed fluid management strategy has been shown to shorten the duration of IMV (Level 2).

\section{Recommendations}

59. Treating the precipitant cause of AHRF, normalising $\mathrm{pH}$, correcting chronic hypercapnia and addressing fluid overload should all occur before starting weaning (Grade D).

60. A BNP-directed fluid management strategy should be considered in patients with known left ventricular dysfunction (Grade B).

\section{Weaning methods}

Despite several multinational studies, there is no consensus as to the optimal weaning method. Brochard et $a l^{232}$ reported that progressively reducing PS was better than other weaning methods. Subsequent trials have reported that daily (or multiple) $\mathrm{T}$ piece trials (SBTs) are as effective as PS weaning. ${ }^{233}{ }^{234} \mathrm{It}$ is likely that patient-specific characteristics are more important than the weaning protocol in determining the duration of 
weaning. There is agreement that the Synchronised Intermittent Mandatory Ventilation method is inferior to PS and T piece weaning. It is also accepted that a formalised weaning plan, and staff familiarity with the approach adopted on the ICU, are important factors to improve successful weaning. ${ }^{235}$

\section{Evidence statement}

Progressive reduction of PS and daily SBTs are satisfactory methods of weaning (Level 1+).

\section{Recommendations}

61. Assessment of the readiness for weaning should be undertaken daily (Grade B).

62. A switch from controlled to assisted IMV should be made as soon as the patient recovery allows (Grade C).

63. IMV patients should have a documented weaning plan (Grade B).

\section{Assessing readiness for discontinuation of mechanical ventilation}

SBTs are used to assess readiness to resume normal breathing. During the SBT, a patient breathes with minimal or no PS (defined as <8). A successful trial requires the absence of respiratory distress. Failure of an SBT may be defined by subjective (comfort) or objective (deterioration in gas exchange or measured ventilator parameters) criteria. ${ }^{232} 233$ Studies have shown that the majority of SBT failures occur within $30 \mathrm{~min}^{236} 237$ Repeated failure of SBT should lead to consideration of other methods of weaning. 238239

It is important to note that the criteria that define success of an SBT do not necessarily reflect the likelihood of successful extubation. About $10 \%$ of patients who successfully manage an SBT will fail to maintain adequate gas exchange and/or develop signs of distress following extubation. ${ }^{240}$ An SBT assesses the balance of respiratory load to capacity of the respiratory muscles but does not take into account other factors that may affect success such as upper airway patency, bulbar function, sputum load or effectiveness of cough. ${ }^{240}$

Evidence statement

A SBT is useful in assessing load/capacity but does not predict the success of extubation (Level 1+).

\section{Recommendation}

64. A 30 min SBT should be used to assess suitability for extubation (Grade B).

65. Factors including upper airway patency, bulbar function, sputum load and cough effectiveness should be considered prior to attempted extubation (Grade D).

\section{Outcome following extubation}

Successful extubation is defined as the absence of the need for ventilatory support for $48 \mathrm{~h}$. Patients receiving post-extubation NIV (see below) are classified as 'weaning in progress'. ${ }^{241}$

Much of the evidence regarding the prediction of the risk of post-extubation failure has come from trials of relatively short duration IMV and with a mixture of underlying pathologies. $^{235} 242243$ Several risk factors have been identified. The more adverse factors present, the greater the risk of extubation failure. Risk factors for extubation failure are shown in box 2.235 242243

Respiratory distress may occur early or develop later on after extubation. Early failure commonly results from loss of airway patency, for example, from upper airway oedema that becomes evident following removal of the endotracheal tube. ${ }^{244}$ Patients with NMD are at risk of early extubation failure due to bulbar dysfunction and/or ineffective cough despite a successful SBT. The planned use of NIV and an MI-E following extubation reduces the risk of early failure. Late extubation failure is more complex in aetiology and more than one cause may be present. The causes are summarised below: ${ }^{244}$

- Capacity-load imbalance: patients with severe airflow obstruction or neuromuscular weakness;

- Impaired bulbar function: aspiration of upper airway secretions, impaired gas exchange and/or obstructed breathing;

- Ineffective cough: typically in NMD/CWD but also in other patients with AHRF;

- Non-respiratory issues-myocardial ischaemia/left ventricular dysfunction, encephalopathy/delirium or severe abdominal distension.

Evidence statement

Patient, clinical and ventilatory factors aid the identification of patients at increased risk of extubation failure (Level $2+$ ).

Recommendation

66. Care is needed to identify factors that increase the risk of extubation failure so that additional support, such as NIV or cough assist, can be provided (Grade B).

\section{Weaning protocols}

Weaning protocols that specify the steps to follow during weaning have been claimed to reduce the duration of IMV, increase the success of extubation, reduce unplanned or accidental extubation and reduce the tracheostomy rate, ventilatorassociated complications and costs, compared with usual care. ${ }^{245}$ The studies summarised in this review were, however, not specific to AHRF. Most were performed in the USA, where differences in supervision of patient management exist compared with the UK. There is also marked variation in the weaning methods and protocols between the studies reported. A European study reported that a weaning protocol did not reduce ventilation time. ${ }^{246}$ Computer-automated weaning, in which adjustment in pressure settings occurs in response to changes in patient parameters, has been compared to professional-led weaning. One multicentre RCT found that duration of weaning was reduced. ${ }^{247}$ A second study reported no difference in weaning duration between automated weaning and weaning by an experienced nurse. ${ }^{248}$ There is currently insufficient evidence to support the use of automated weaning over clinical/nurse-led protocols.

\section{Evidence statements}

Weaning protocols may reduce the duration of IMV and ventilator associated pneumonia (Level 1+).

There is conflicting evidence regarding the value of computerautomated weaning (Level 1-).

\section{Box 2 Risk factors for extubation failure following} invasive mechanical ventilation (IMV)

- Positive fluid balance

- Raised rapid shallow breathing index during spontaneous breathing trial

- Pneumonia or pulmonary disease as the cause requiring IMV

- Increased age

- Prolonged duration of IMV

- Anaemia

- Increased severity of illness

- Low albumin

- Previous failed extubation

- Bulbar dysfunction 


\section{Recommendations}

67. Although an organised and systematic approach to weaning is desirable, protocols should be used with caution in patients with AHRF (Grade B).

68. The use of computerised weaning cannot be recommended in AHRF (Grade D).

\section{Use of NIV in the ICU}

Planned NIV to speed weaning from IMV

In an uncontrolled study on lung transplantation, NIV was found to speed extubation and reduce the time spent invasively ventilated and the attendant complications. ${ }^{249}$ Subsequent studies have compared the use of NIV with conventional weaning in patients who have failed an SBT. Benefit was demonstrated in patients with underlying COPD. ${ }^{50} 250$ These studies utilised NIV at high levels of PS and for longer than $24 \mathrm{~h}$. NIV weaning was reported to confer no benefit in a subsequent study. ${ }^{251}$ A subsequent Cochrane review concluded that the use of NIV to speed weaning in patients with COPD reduced mortality and the incidence of pneumonia without increasing the need for re-intubation. ${ }^{252}$

Evidence statements

NIV has been shown to accelerate weaning from IMV in the patient with COPD failing an SBT (Level 1+).

\section{Recommendation}

69. NIV is recommended to aid weaning from IMV in patients with AHRF secondary to COPD (Grade B).

70. In other causes of AHRF, NIV may have a role in shortening the duration of IMV when local expertise in its use exists (and of cough assist when indicated) and there are features present that indicate extubation is likely to be successful (Grade D).

\section{NIV in high-risk patients}

NIV has been assessed in patients who have passed an SBT but who have risk factors for extubation failure such as age $>65$ years, poor cough, cardiac and respiratory comorbidity, and hypercapnia (while ventilated and/or pre-existing). NIV was reported to reduce the re-intubation rate and mortality in one study, ${ }^{253}$ and has been reported to be effective where obesity $\left(\mathrm{BMI}>35 \mathrm{~kg} / \mathrm{m}^{2}\right)$ is an additional adverse feature. ${ }^{254}$

Evidence statements

NIV may be effective in reducing respiratory failure, re-intubation and mortality in COPD (Level $1+$ ) and patients with increased BMI (Level 2+).

Planned post-extubation NIV reduces mortality, ICU and hospital length of stay and the incidence of ventilator-associated pneumonia (Level 1-).

\section{Recommendation}

71. Prophylactic use of NIV should be considered to provide post-extubation support in patients with identified risk factors for extubation failure (Grade B).

\section{NIV as 'rescue' therapy post-extubation}

A number of RCTs have examined the use of NIV as an unplanned 'rescue' treatment for post-extubation respiratory distress. One multicentre RCT reported that patients who passed an SBT but who then developed post-extubation respiratory failure had an increased ICU mortality rate if treated with NIV as opposed to re-intubation. ${ }^{181}$ This study has been criticised as few patients were treated in each participating centre, despite a long recruitment period, raising the suspicion that lack of familiarity with NIV may have resulted in it being poorly applied. The patients who failed NIV and went on to require intubation also received long periods of ineffective NIV before re-intubation, $9 \mathrm{~h}$ longer than the control group. This may have contributed to the worse outcome. Post hoc analysis suggested a benefit with NIV post-extubation in patients with COPD.

Evidence statement

The use of NIV as rescue therapy for unexpected postextubation respiratory failure does not improve outcome and may be detrimental (Level 1+).

Recommendations

72. NIV should not be used routinely for unexpected postextubation respiratory failure (Grade B).

73. In COPD, a trial of NIV may be justified for unexpected post-extubation respiratory failure where local expertise exists (Grade D).

\section{CARE PLANNING AND DELIVERY OF CARE}

\section{Appropriate care environments for the delivery of NIV}

A study by Roessler and colleagues from Germany randomised 51 patients to either out-of-hospital NIV or standard medical treatment. Out-of-hospital NIV was reported to be feasible, safe and effective. ${ }^{255}$ A survey of French mobile ICUs also suggests that NIV and CPAP can be safely employed pre-hospital in acute cardiogenic pulmonary oedema but not in other causes of respiratory failure. ${ }^{256}$ Further evaluation of out-of-hospital NIV in AHRF is required.

NIV is commonly initiated in the ED, but given the other priorities and pressures on emergency resuscitation areas patients should be transferred as soon as practicable to an environment appropriately staffed and equipped to provide on going care. A prospective observational study of 245 patients attending 24 hospital EDs in Australia identified the staff responsible for NIV set-up. ${ }^{257}$ This was equally distributed between nursing and medical personnel. Hess et $\mathrm{al}^{258}$ conducted a survey of 132 academic EDs in the USA, and concluded that, although NIV was widely available, physician confidence/competence was a barrier to optimal application. A survey of NIV use in UK EDs found a wide variety of practice and suggested the need for a specific ED guide for NIV. ${ }^{259}$ A pro forma-based COPD management tool, supported by targeted education, was reported to improve ED care including the use of NIV. ${ }^{260}$

Previous guidelines have recommended limiting the number of areas providing NIV to ensure that staff perform it sufficiently regularly. ${ }^{48}$ Suitable sites need to be able to provide an NIV service $24 / 7$ and integration with critical care services is essential. If NIV is provided in more than one area within a hospital, protocols and guidelines should be shared. ${ }^{261}$ Current NICE Quality Standards for COPD include guidance on how to benchmark NIV provision. ${ }^{262}$ The requirements for an NIV service are summarised in box 3 .

For all but the mildest cases, Nava and Hill ${ }^{263}$ recommend that NIV be delivered in a level 2 facility with enhanced staffing levels. A survey carried out in 1999 found that NIV was provided in level 2/3 facilities in most western European countries. ${ }^{264}$ In contrast, NIV has been delivered in admission or respiratory ward settings in the UK. This may partially account for the poor performance and high mortality rates associated with use of NIV reported by audits. ${ }^{3-5}$ The 2009 ICS recommendations ${ }^{265}$ reiterate level 2 as the appropriate clinical environment for NIV and the 2008 joint BTS, ICS and RCP guide on the use of NIV in COPD with $\mathrm{AHRF}^{266}$ recommends one nurse for every 2 NIV cases, especially during the first $24 \mathrm{~h}$ of treatment.

Despite this, in the 2013 BTS NIV audit, 91\% of patients were treated on general medical wards despite $43 \%$ having $\mathrm{pH}<7.25$. $^{5}$ This was associated with a low intubation rate and 


\section{Box 3 Essential requirements for an NIV service}

- Specifically identified area(s) for NIV treatment at level 2 equivalence.

- Staffing levels above that of a general medical ward with one nurse for every 2 NIV cases (especially during the first $24 \mathrm{~h}$ of treatment)

- Locally developed NIV protocols (based on published best practice guides) uniformly applied across all areas

- A designated lead with a 'core' multidisciplinary group (physicians, nurses, physiotherapists) co-ordinating NIV service provision and linked with critical care services

- Access to expert support for NIV technical advice in and out of hours

- Mechanisms for regular audit

- Regular staff educational updates and training module for new staff

excess mortality. Although the care plan in $21 \%$ of cases included IMV should NIV fail, only $3 \%$ were intubated (versus an expected rate of $7 \%$ ). Overall, the audit found that mortality in the AECOPD group was $28 \%$ if NIV was delivered in HDU/ ICU and $40 \%$ if not. With a median $\mathrm{pH}$ of 7.24 for the whole patient population (2693 cases), this suggests that some were not being treated for lesser degrees of acidosis, where NIV success is more guaranteed, and that those receiving NIV were not placed in the appropriate care environment given the severity of acidosis.

A number of strategies have been explored to support the effective use of NIV outside the HDU/ICU. Sala et $a l^{267}$ described the practicalities of creating a respiratory intermediate care unit. Paus-Jenssen $e t a l,{ }^{268}$ in a Canadian prospective study, used an expert respiratory therapist team to implement NIV across a number of clinical environments. In a similar study, critical care outreach nurses supported NIV delivery elsewhere in the hospital. As a result, mortality was reduced from $57 \%$ to $35 \% .{ }^{183}$ A greater number of patients were also identified as suitable for IMV when failing NIV. Some of the challenges of care delivery in this field are highlighted in the National COPD Audit Programme 2014 findings on resources and organisation of care in acute National health Service (NHS) units, ${ }^{269}$ where only $30 \%$ of outreach programmes operated out of hours during weekdays and 59\% of respiratory wards reported no level 2 capability. Cabrini reported an Italian prospective study of NIV administered in a non-ICU setting but managed by an anaesthetist-led medical team. ${ }^{270}$ In 129 consecutive treatments, $10 \%$ required intubation and there was a low mortality rate of $12.4 \%$. These reports together suggest that collaboration between admitting teams and the ICU can improve the delivery of care in AHRF.

Hospitalisation with AHRF involves 3 phases-immediate clinical assessment, an assisted ventilation plan when appropriate and the formulation of a future care plan (short term in the event of NIV failure and long term on recovery and discharge or, depending on progress, the provision of end of life care). Figure 3 details key elements and box 4 provides a discharge checklist.

It has been estimated that an average-sized district general hospital, serving a population of 250000 , should anticipate, depending on local COPD prevalence, up to 100 AECOPD cases requiring ventilatory support per annum. Given the additional causes of AHRF, this probably equates to $150 \mathrm{NIV/IMV}$ cases in most hospitals, and considerably more in areas with high COPD and/or OHS prevalence or those hospitals serving larger populations. NIV facilities should be able to cope with seasonal variation and the increased demand that may occur during influenza epidemics. ${ }^{271}$

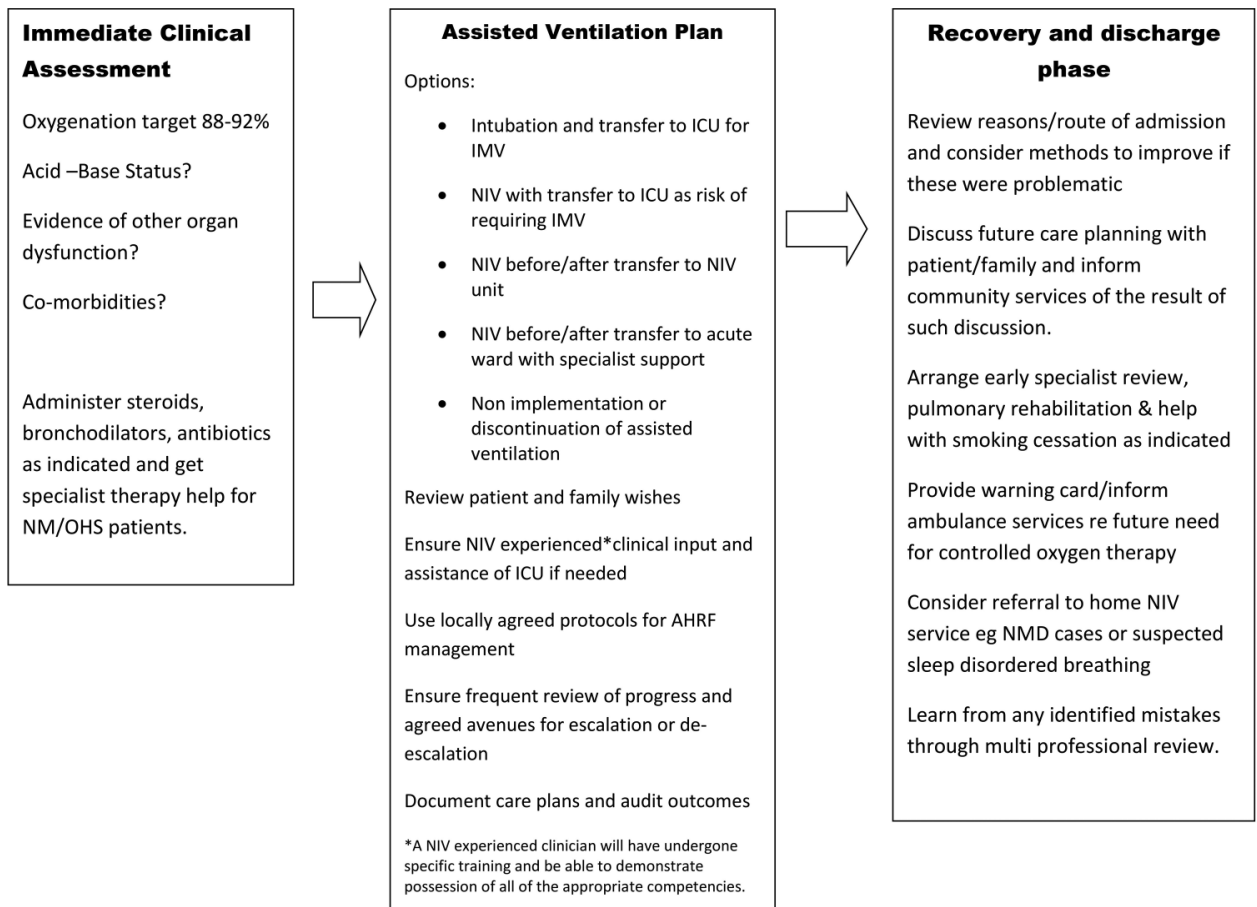

Figure 3 The three phases of patient management in acute hypercapnic respiratory failure. 
As discussed in the Management section, patient outcomes reported in UK national audits are notably worse than would be expected from trial data and facilities for provision of NIV, and evidence of consultation with the ICU, are frequently limited or inadequate. ${ }^{3} 47$ Important deficiences that have been identified include delays in commencing ventilatory support, under-recognition of more complex acid-base disturbances, use of inadequate ventilatory pressures, rare use of a different mask when NIV is failing, lack of progression from NIV to IMV and lack of consultation in decision-making. The preponderance for application of NIV in lower level facilities than elsewhere in the world (outlined above) along with evidence of a lack of integration with the ICU, ${ }^{3}{ }^{4}$ indicates that attention directed at organisational factors are needed and are highly likely to improve patient outcome and experience in AHRF.

NIV facilities need to encompass adequate capacity, and the expertise and associated staffing levels, to deal with complex critically ill patients who have a significant risk of death. To be effective, the NIV service needs to have good operational links to the ICU in the expectation that $10 \%$ to $20 \%$ of NIV-treated patients should be managed in HDU/ICU and that many will be potential candidates for IMV. The case for a specifically identified and appropriately staffed and equipped area for providing NIV is strongly supported by the evidence. In some European countries, NIV services are provided in a Respiratory Intermediate Care Unit. ${ }^{264}$

Evidence statements

A care environment with either level 2 or 3 staffing favours a successful outcome from NIV therapy (Level 2+).

Coordination between the ICU and ward areas improves outcome in AHRF (Level 3).

Organisational aspects are pivotal in achieving best outcomes (Level 4).

Recommendations

74. NIV services should operate under a single clinical lead with formal working links with the ICU (Grade D).

75. The severity of AHRF, and evidence of other organ dysfunction, should influence the choice of care environment (Grade C) .

76. NIV should take place in a clinical environment with enhanced nursing and monitoring facilities beyond those of a general medical ward (Grade C).

\section{Box 4 Discharge checklist after AHRF}

- Arrange early specialist review, pulmonary rehabilitation and help with smoking cessation as indicated.

- Consider early home visit, for example, outreach COPD team/community nurses

- Discuss future care planning with patient/family and inform community services of the result of such discussion

- Provide warning card/inform ambulance services regarding future need for controlled oxygen therapy

- Consider referral to home NIV service, for example, neuromuscular disease (NMD) cases or suspected sleep disordered breathing

- Review reasons/route of admission and consider methods to improve if these were problematic

- Learn from any identified mistakes through multiprofessional review
77. Initial care plans should should include robust arrangements for escalation, anticipating that up to $20 \%$ of AHRF cases should be managed in a level 2 or 3 environment (Grade C).

Good practice points

- A 2-4 bedded designated NIV unit (located within a medical high dependency area or within a respiratory ward with enhanced staffing levels) provides a robust basis for the provision of NIV in a DGH serving a population of 250000 and with an average prevalence of COPD.

- Areas providing NIV should have a process for audit and interdisciplinary communication.

\section{Palliative care and advanced care planning}

It is recognised that palliative interventions may be appropriate and yet be provided at the same time as therapies intended to prolong life. ${ }^{272}$ Accordingly, employing NIV as part of care that aims to relieve distress and has escalation limits may be entirely justified.

Effort is needed to establish patient preferences with respect to intubation or resuscitation status. Momen et al, ${ }^{273}$ in a systematic review of end-of-life conversations in COPD, found considerable variation among patients in the desire to discuss end of life. Almost $50 \%$ of patients did not wish to have such a conversation and there was a preference to wait until the disease was 'advanced', with patient perception that this implied the last few days of life. Advance directives/living wills assist healthcare providers in tailoring clinical response and support. ${ }^{274}$ The importance of actively involving patient/family, especially regarding 'do not attempt cardiopulmonary resuscitation' (DNACPR) orders, are highlighted in revised recommendations following a judicial ruling. ${ }^{275}$ The essential element is that, while patients cannot insist on CPR being performed, the matter should be discussed. Perceived patient 'distress' - which might be exacerbated by such discussion-is no longer regarded sufficient grounds for not raising the issue. When the risk of causing physical or psychological harm is present, attempts should be made to talk to a healthcare advocate. The enormous challenges in this serve to emphasise the crucial nature of active and ongoing communication strategies. Chakrabarti reported interviews with 50 patients with stable COPD and found that discussion and demonstration of NIV equipment altered future treatment perceptions and willingness to consider an advance directive. $^{276}$

Sinuff $e t a l^{277}$ reported clinician attitudes to NIV in patients with acute respiratory failure and do not intubate/do not resuscitate instructions. While about $60 \%$ of physicians considered that NIV should be discussed in this context, $85 \%$ of respiratory therapists (those actually administering NIV) felt NIV should be actively promoted. This may reflect a lack of confidence and understanding, among physicians, of the potential for NIV to relieve distress and be effective even in advanced disease. In Denmark, $15 \%$ of patients with do not intubate instructions, and who received NIV, survived at least a year with COPD and congestive heart failure the most favourable underlying diagnoses. ${ }^{278}$

\section{Evidence statements}

In advanced disease, care planning should ideally predate acute presentation or commence as early as possible on presentation with AHRF (Level 4).

Health professionals experienced in NIV delivery have a more positive view of the benefit of NIV and perceive patient treatment wishes more postively than do clinicians with less experience of NIV (Level 4). 


\section{Recommendations}

78. Clinicians delivering NIV or IMV should have ready access to palliative medicine (Grade D).

79. Multidisciplinary advance care planning should be an integral part of the routine outpatient management of progressive or advanced disease and care plans should be reviewed on presentation during an episode of AHRF (Grade D).

80. The use of NIV may allow time to establish patient preference with regard to escalation to IMV (Grade D).

\section{End-of-life care}

A questionnaire study of 118 patients with COPD, carried out in Canadian teaching hospitals, ${ }^{279}$ reported that patients with COPD were less interested in prognosis, CPR, IMV or referral to palliative care than were patients with metastatic cancer. In another study, comparing QoL between patients with advanced COPD and patients with cancer, patients with COPD reported higher levels of physical discomfort with uncontrolled shortness of breath in $78 \% .{ }^{280} \mathrm{~A}$ recent review of the 4 RCTs that have explored whether NIV relieves dyspnoea in AECOPD concluded that benefit was likely but that study limitations constrained a confident conclusion. ${ }^{281}$ With regard to physical symptoms, breathlessness and fatigue are dominant in AECOPD. Attention to secretion clearance is an additional major concern in bronchiectasis and CF and for many with NMD. Ability to communicate, to feel safe, to be individually respected and enabled to retain control are common psychological needs.

Patients receiving NIV as 'ceiling care', who fail to improve will need appropriate end-of-life attention, including appropriate sedation/relief of distress. It is important that if withdrawal of NIV is decided on, that this is achieved with minimum distress to the patient and their relatives. The BMA guidance on end-of-life care in 2007 did not address withdrawal of assisted ventilation. ${ }^{282}$ Although withholding and withdrawing are considered ethically equivalent, ${ }^{283}$ for many individuals, including clinicians, discontinuing mechanical ventilation is felt be emotionally different to, for instance, stopping haemodialysis. This may be because of the immediacy of the consequence. ${ }^{284} \mathrm{~A}$ Japanese study reported interviews with 35 critical care physicians and found withdrawing ventilation was regarded differently to stopping other life-sustaining measures because of concern over an abrupt and distressing demise. ${ }^{285}$ There was a desire for a 'soft landing', with a slow and gradual death perceived as 'natural'. The ATS Clinical Policy Statement of 2007 provides comprehensive guidance on withdrawal of mechanical ventilation, including symptom management of the dying patient. It emphasises that decision-making is a process requiring frequent discussion with patient, family, health professionals and others. ${ }^{286}$ Pro-active family-centred conferences allow time for families to adjust and provision of literature on bereavement reduces the risk of subsequent emotional morbidity. ${ }^{287}$

In practical terms, progressive reduction of pressure/backup rate to achieve $\mathrm{CO}_{2}$ narcosis/coma and an alternative strategy of extubation when intubated or removal of NIV have both been described. In the former scenario, Cox et al ${ }^{288}$ suggest initial weaning of oxygen over $10 \mathrm{~min}$ with appropriate adjustment to opiate or anxiolytic medication. Once patient comfort is assured, it is suggested that mandatory ventilation is withdrawn and PS reduced to zero over 5-10 min. Kuhnlein et $a l^{289}$ conducted structured interviews with 29 families regarding the circumstances of dying in MND patients receiving NIV. Seventeen caregivers described the final stages and eventual death as 'peaceful'. Eleven of the patients died peacefully while using NIV. Choking sensation was evident in some bulbar patients.
The authors indicated that the use of sedatives, anxiolytics and opiates could have been improved, emphasising that palliative care training or support is needed to achieve best practice.

In conclusion, the role of NIV in achieving a 'good death' may currently be underutilised and there may be a lack of appreciation that a peaceful death can occur while receiving supportive ventilation.

Evidence statements

The concerns of patients with COPD towards their end of life, centre on high levels of physical symptoms, especially breathlessness (Level 3).

Clinicians often consider withdrawal of assisted ventilation (NIV/IMV) as more challenging than removal of other life support techniques (Level 4).

Good practice points

- Although removal of the NIV mask may be deemed as preferable, a dignified and comfortable death is possible with it in place.

- Clinicians delivering NIV or IMV should have training in end-of-life care and the support of palliative care teams.

\section{NOVEL THERAPIES}

\section{Extracorporeal $\mathrm{CO}_{2}$ removal}

The technical aspects of providing prolonged extracorporeal membrane oxygenation (ECMO) or $\mathrm{CO}_{2}$ removal $\left(\mathrm{ECCO}_{2} \mathrm{R}\right)$ have advanced in recent years. Both are being increasingly investigated in refractory respiratory failure including AHRF. NICE has issued general guidance on the use of $\mathrm{ECCO}_{2} \mathrm{R}$ advising that it should only be used in patients with potentially reversible hypercapnic respiratory failure or those being considered for lung transplantation. ${ }^{290}$

$\mathrm{ECCO}_{2} \mathrm{R}$ uses a gas exchange membrane to provide partial $\mathrm{CO}_{2}$ clearance, from $30 \%$ to $50 \%$ of the body's production, depending on blood flow and membrane efficiency. Removing carbon dioxide extracorporeally reduces the native pulmonary minute ventilation required to maintain an acceptable $\mathrm{PaCO}_{2}$. This offers the potential benefits of either enabling protective mechanical ventilation or providing an alternative to mechanical ventilation in selected patients (such as those with COPD). There is little evidence of clear benefits to patients of $\mathrm{ECCO}_{2} \mathrm{R}$ at present. In moderately hypoxaemic ARDS, one RCT demonstrated that lower tidal volumes and ventilatory pressures could be achieved, but this failed to translate into a meaningful improvement in patient outcome. ${ }^{291}$ Larger studies are planned in the UK and Europe. In patients with COPD, there are no RCTs exploring the role of $\mathrm{ECCO}_{2} \mathrm{R}$. One retrospectively matched cohort study compared outcomes between groups of patients with AECOPD who had an inadequate response to NIV. Twenty five potential candidates who had failed to improve with NIV were compared with historical controls treated in the same hospitals matched by the GenMatch process. Despite significant improvements in acidosis and respiratory distress, the trial failed to show benefit in the primary outcome of need for intubation. The complication rate with $\mathrm{ECCO}_{2} \mathrm{R}$ was high (52\%) and this contributed to the need for intubation. ${ }^{292}$

The devices available for $\mathrm{ECCO}_{2} \mathrm{R}$ have evolved over time. Early $\mathrm{CO}_{2}$ removal membranes were pumpless, required arterial and venous cannulation and used the patients own cardiac output to drive blood through the membrane. This resulted in significant shunting of cardiac output and the danger of limb ischaemia. An alternative approach is to take blood from a duallumen large bore cannula sited in a central vein and pump it through the membrane. The advantages of the veno-venous 
technique are principally lack of effect on cardiac output and reduced complications, particularly limb ischaemia.

Evidence statements

Extra-corporeal $\mathrm{CO}_{2}$ removal devices can reduce $\mathrm{PaCO}_{2}$ and minute volume (Level 2-).

Veno-venous extra-corporeal $\mathrm{CO}_{2}$ removal in patients with AECOPD and an inadequate response to NIV has not been shown to reduce intubation rate and is associated with a $52 \%$ complication rate (Level 2-).

\section{Recommendations}

81. If local expertise exists, $\mathrm{ECCO}_{2} \mathrm{R}$ might be considered:

- If, despite attempts to optimise IMV using lung protective strategies, severe hypercapnic acidosis $(\mathrm{pH}<7.15)$ persists (Grade D);

- When 'lung protective ventilation' is needed but hypercapnia is contraindicated, for example, in patients with coexistent brain injury (Grade D);

- For IMV patients awaiting a lung transplant (Grade D).

Good practice point

$\mathrm{ECCO}_{2} \mathrm{R}$ is an experimental therapy and should only be used by specialist intensive care teams trained in its use and where additional governance arrangements are in place or in the setting of a research trial.

\section{Helium/oxygen ventilation}

When mixed with oxygen (Heliox), the lower density of helium reduces resistance in the large airways where flow is predominantly turbulent compared to air/oxygen ventilation. It therefore has a theoretical advantage in obstructive causes of AHRF. ${ }^{293}$ Heliox has been studied in combination with both NIV and IMV. It increases the delivered dose of bronchodilators and has been reported to improve symptoms and physiological variables in spontaneously breathing asthmatics. ${ }^{294} 295$ At oxygen concentrations $>40 \%$, Heliox has no benefit compared with oxygenair mixtures. ${ }^{296}$ A large RCT in AECOPD found that Heliox in combination with NIV did not reduce rates of intubation, duration of ventilatory support or mortality. ${ }^{297}$ Heliox has been reported to reduce $\mathrm{pCO}_{2}$ and airway pressures in intubated patients with severe asthma ${ }^{298}$ but a subsequent meta-analysis concluded that it did not affect outcome. ${ }^{299}$ An uncontrolled study reported that Heliox improved patient comfort in the presence of post-extubation respiratory distress when stridor was present. ${ }^{300}$

Evidence statement

The use of Heliox does not reduce rates of intubation and length of IMV, nor does it reduce mortality in patients of AECOPD or asthma (Level 1+).

\section{Recommendation}

82. Heliox should not be used routinely in the management of AHRF (Grade B).

\section{Author affiliations}

'British Thoracic Society, London, UK

${ }^{2}$ St James's University Hospital, Leeds, UK

${ }^{3}$ Barts Health NHS Trust, London, UK

${ }^{4}$ Department of Respiratory Research, University Hospitals of Coventry and

Warwickshire NHS Trust, Coventry, UK

${ }^{5}$ Sheffield Teaching Hospitals NHS Foundation Trust, Sheffield, UK

${ }^{6}$ Department of Respiratory, Scottish Pulmonary Vascular Unit, Glasgow, UK

${ }^{7}$ Royal Surrey County Hospital NHS Foundation Trust and Faculty of Health and

Medical Sciences, University of Surrey, Guildford, UK

${ }^{8}$ Academic Respiratory Unit, University of Bristol, Bristol, UK

${ }^{9}$ North Bristol Lung Centre, North Bristol NHS Trust, Bristol, UK

${ }^{10}$ University Hospital of South Manchester NHS Foundation Trust, Manchester, UK

${ }^{11}$ Emergency Department, Manchester Royal Infirmary, Central Manchester University Hospitals NHS Foundation Trust, Manchester, Manchester, UK M13 9WL
}

${ }^{12}$ University of Plymouth, Plymouth, UK

${ }^{13}$ Department of Physiotherapy, Guy's and St Thomas' NHS Foundation Trust, St

Thomas' Hospital, London, UK

${ }^{14}$ Aintree University Hospital, Liverpool, UK

${ }^{15}$ CLAHRC, Imperial College, London, UK

${ }^{16}$ Queen's Medical Centre, Nottingham, UK

${ }^{17}$ Royal College of Physicians, London, UK

Twitter Follow BTS at @BTSrespiratory, Ben Creagh-Brown at @SPACeRGroup and James Dodd at @theotherdodd

Acknowledgements The authors acknowledge assistance with developing the aims and structure of the guidelines provided by Martin Wildman, Simon Baudouin, Robert Winter, Sara Bolton, Mandy Odell, David Smith and the members of the BTS Standards of Care Committee.

Collaborators BTS Standards of Care Committee members: Colin Gelder, James Dodd and Caroline Patterson.

Contributors $A C D$ chaired the guideline group, and led the drafting and revision of the document. He has final responsibility for the guideline. SB, ME, DK, CG and AG drafted and revised the paper. CC, BC-B, JD, TF, BF, LM, LMCD, RP, CP, MS and $\mathrm{LT}$ conducted appraisal of the literature and provided draft sections of the document.

Representation Dr Bob Winter and Sara Bolton represented the Intensive Care Society, Dr Bernard Foëx represented the College of Emergency Medicine, Dr Daniel Kennedy represented the Royal College of Anaesthetists and Surgeon Captain Lynn Thomas represented the Royal College of Physicians.

Competing interests $A C D$ declares being paid as a consultant to Smith Medical between 2008 and 2013. ME declares he has received an honorarium, and travel and subsistence expenses for speaking at a meeting in Australia organised by Resmed, a Respiratory Sleep and Ventilation company. He has received an honorarium and travel expenses for speaking at a meeting in London organised by Phillips Respironics, a Respiratory Sleep and Ventilation company. He has received travel and subsistence expenses for speaking at a meeting in China organised by Curative Medical Inc, a Respiratory Sleep and Ventilation company. He has received travel expenses for speaking at meetings in India organised by Phillips Respironics, a Respiratory Sleep and Ventilation company. AG declares being paid as a consultant and receiving honoraria and travel expenses for speaking at meetings organised by Armstrong Medical Ltd in the UK, between 2014 and 2015.

Provenance and peer review Not commissioned; internally peer reviewed.

\section{REFERENCES}

1 British Thoracic Society Standards of Care Committee. Non-invasive ventilation in acute respiratory failure. Thorax 2002;57:192-211.

2 Plant PK, Owen J, Elliott MW. One year period prevalance study of respiratory acidosis in acute exacerbation of COPD; implications for the provision of non-invasive ventilation and oxygen administration. Thorax 2000; 55:550-4.

3 Davidson AC. Report British Thoracic Society NIV Audit 2011. https://www. brit-thoracic.org.uk/document-library/audit-and-quality-improvement/audit-reports/ bts-adult-niv-audit-report-2011/

4 Davies M. Report British Thoracic Society NIV Audit 2012. https://www. brit-thoracic.org.uk/document-library/audit-and-quality-improvement/audit-reports/ bts-adult-niv-audit-report-2012/

5 Davies M. Report British Thoracic Society NIV Audit 2013. https://www. brit-thoracic.org.uk/document-library/audit-and-quality-improvement/audit-reports/ bts-adult-niv-audit-report-2013/

6 Davidson AC. Towards a comprehensive ventilatory strategy for acute exacerbations of COPD. JICS 2008:9:5-7.

7 Roberts CM, Stone RA, Buckingham RJ, et al. Acidosis, non-invasive ventilation and mortality in hospitalised COPD exacerbations. Thorax 2011;66:43-8.

8 Gupta D, Keogh B, Chung KF, et al. Characteristics and outcome for admissions to adult, general critical care units with acute severe asthma: a secondary analysis of the ICNARC Case Mix Programme Database. Crit Care 2004;8:R112-21.

9 Hull J, Aniapravan R, Chan E, et al. British Thoracic Society guideline for respiratory management of children with neuromuscular weakness. Thorax 2012;67(Suppl 1):i1-40.

10 British Thoracic Society. BTS quideline production manual. London: BTS, 2014

11 AGREE. AGREE II Instrument. Secondary AGREE II Instrument 2010. http://www. agreetrust.org/agree-ii/

12 Mehta S, McCool FD, Hill NS. Leak compensation in positive pressure ventilators: a lung model study. Eur Respir J 2001;17:259-67.

13 Storre JH, Bohm P, Dreher M, et al. Clinical impact of leak compensation during non-invasive ventilation. Respir Med 2009:103:1477-83.

14 Ferguson GT, Gilmartin M. CO2 rebreathing during BiPAP ventilatory assistance. Am J Respir Crit Care Med 1995;151:1126-35. 
15 Szkulmowski Z, Belkhouja K, Le QH, et al. Bilevel positive airway pressure ventilation: factors influencing carbon dioxide rebreathing. Intensive Care Med 2010;36:688-91.

16 Ram FS, Picot J, Lightowler J, et al. Non-invasive positive pressure ventilation for treatment of respiratory failure due to exacerbations of chronic obstructive pulmonary disease. Cochrane Database Syst Rev 2004;(3):CD004104.

17 Lofaso F, Brochard L, Hang T, et al. Home versus intensive care pressure support devices. Experimental and clinical comparison. Am J Respir Crit Care Med 1996;153:1591-9.

18 Schettino GP, Tucci MR, Sousa R, et al. Mask mechanics and leak dynamics during noninvasive pressure support ventilation: a bench study. Intensive Care Med 2001:27:1887-91.

19 Tassaux D, Strasser S, Fonseca S, et al. Comparative bench study of triggering, pressurization, and cycling between the home ventilator VPAP II and three ICU ventilators. Intensive Care Med 2002;28:1254-61.

20 Battisti A, Tassaux D, Janssens JP, et al. Performance characteristics of 10 home mechanical ventilators in pressure-support mode: a comparative bench study. Chest 2005; 127:1784-92.

21 Vignaux L, Tassaux D, Carteaux G, et al. Performance of noninvasive ventilation algorithms on ICU ventilators during pressure support: a clinical study. Intensive Care Med 2010;36:2053-9.

22 Olivieri C, Costa R, Conti G, et al. Bench studies evaluating devices for non-invasive ventilation: critical analysis and future perspectives. Intensive Care Med 2012;38:160-7.

23 Ferreira JC, Chipman DW, Hill NS, et al. Bilevel vs ICU ventilators providing noninvasive ventilation: effect of system leaks: a COPD lung model comparison. Chest 2009;136:448-56.

24 Carteaux G, Lyazidi A, Cordoba-Izquierdo A, et al. Patient-ventilator asynchrony during noninvasive ventilation: a bench and clinical study. Chest 2012;142:367-76.

25 Lien TC, Wang JH, Huang SH, et al. Comparison of bilevel positive airway pressure and volume ventilation via nasal or facial masks in patients with severe, stable COPD. Zhonghua Yi Xue Za Zhi (Taipei) 2000;63:542-51.

26 Anton A, Tarrega J, Giner J, et al. Acute physiologic effects of nasal and full-face masks during noninvasive positive-pressure ventilation in patients with acute exacerbations of chronic obstructive pulmonary disease. Respir Care 2003;48:922-5.

27 Corbetta L, Ballerin L, Putinati S, et al. Efficacy of noninvasive positive pressure ventilation by facial and nasal mask in hypercapnic acute respiratory failure: experience in a respiratory ward under usual care. Monaldi Arch Chest Dis 1997;52:421-8

28 Cuvelier A, Pujol W, Pramil S, et al. Cephalic versus oronasal mask for noninvasive ventilation in acute hypercapnic respiratory failure. Intensive Care Med 2009;35:519-26.

29 Fodil R, Lellouche F, Mancebo J, et al. Comparison of patient-ventilator interfaces based on their computerized effective dead space. Intensive Care Med 2011;37:257-62.

30 Fraticelli AT, Lellouche F, L'Her E, et al. Physiological effects of different interfaces during noninvasive ventilation for acute respiratory failure. Crit Care Med 2009;37:939-45.

31 Holanda MA, Reis RC, Winkeler GF, et al. Influence of total face, facial and nasal masks on short-term adverse effects during noninvasive ventilation. J Bras Pneumol 2009;35:164-73.

32 Navalesi P, Fanfulla F, Frigerio P, et al. Physiologic evaluation of noninvasive mechanical ventilation delivered with three types of masks in patients with chronic hypercapnic respiratory failure. Crit Care Med 2000;28:1785-90.

33 Racca F, Appendini L, Berta G, et al. Helmet ventilation for acute respiratory failure and nasal skin breakdown in neuromuscular disorders. Anesth Analg 2009;109:164-7.

34 Willson GN, Piper AJ, Norman M, et al. Nasal versus full face mask for noninvasive ventilation in chronic respiratory failure. Eur Respir J 2004;23:605-9.

35 Gregoretti C, Confalonieri M, Navalesi P, et al. Evaluation of patient skin breakdown and comfort with a new face mask for non-invasive ventilation: a multi-center study. Intensive Care Med 2002;28:278-84.

36 Conti G, Cavaliere F, Costa R, et al. Noninvasive positive-pressure ventilation with different interfaces in patients with respiratory failure after abdominal surgery: a matched-control study. Respir Care 2007;52:1463-71.

37 Kwok H, McCormack J, Cece R, et al. Controlled trial of oronasal versus nasal mask ventilation in the treatment of acute respiratory failure. Crit Care Med 2003;31:468-73.

38 Vargas F, Thille A, Lyazidi A, et al. Helmet with specific settings versus facemask for noninvasive ventilation. Crit Care Med 2009;37:1921-8.

39 Antonelli M, Pennisi MA, Pelosi $P$, et al. Noninvasive positive pressure ventilation using a helmet in patients with acute exacerbation of chronic obstructive pulmonary disease: a feasibility study. Anesthesiology 2004;100:16-24.

40 Chiumello D, Pelosi P, Carlesso E, et al. Noninvasive positive pressure ventilation delivered by helmet vs. standard face mask. Intensive Care Med 2003;29:1671-9.
41 Navalesi P, Costa R, Ceriana P, et al. Non-invasive ventilation in chronic obstructive pulmonary disease patients: helmet versus facial mask. Intensive Care Med 2007;33:74-81.

42 Pelosi $P$, Severgnini $P$, Aspesi $M$, et al. Non-invasive ventilation delivered by conventional interfaces and helmet in the emergency department. Eur J Emerg Med 2003;10:79-86.

43 Rabitsch W, Schellongowski P, Kostler WJ, et al. Efficacy and tolerability of non-invasive ventilation delivered via a newly developed helmet in immunosuppressed patients with acute respiratory failure. Wien Klin Wochenschr 2003;115:590-4.

44 Tonnelier JM, Prat G, Nowak E, et al. Noninvasive continuous positive airway pressure ventilation using a new helmet interface: a case-control prospective pilot study. Intensive Care Med 2003;29:2077-80.

45 Cavaliere F, Conti G, Costa R, et al. Exposure to noise during continuous positive airway pressure: influence of interfaces and delivery systems. Acta Anaesthesiol Scand 2008;52:52-6.

46 Ozsancak A, Sidhom SS, Liesching TN, et al. Evaluation of the total face mask for noninvasive ventilation to treat acute respiratory failure. Chest 2011;139:1034-41.

47 Saatci E, Miller DM, Stell IM, et al. Dynamic dead space in face masks used with noninvasive ventilators: a lung model study. Eur Respir J 2004;23:129-35.

48 NICE clinical guidance [CG12]. Chronic obstructive pulmonary diseaseManagement of chronic obstructive pulmonary disease in adults in primary and secondary care. 2004. http://www.nice.org.uk/guidance/cg12

49 Diaz GG, Alcaraz AC, Talavera JCP, et al. Noninvasive positive-pressure ventilation to treat hypercapnic coma secondary to respiratory failure. Chest 2005; 127:952-60.

50 Nava S, Ambrosino N, Cini E, et al. Non invasive mechanical ventilation in the weaning of patients with respiratory failure due to chronic obstructive pulmonary disease: a randomized study. Ann Intern Med 1998;128:721-8.

51 Ides K, Vissers D, De Backer L, et al. Airway clearance in COPD: need for a breath of fresh air? A systematic review. COPD 2011;8:196-205.

52 Hill K, Patman S, Brooks D. Effect of airway clearance techniques in patients experiencing an acute exacerbation of chronic obstructive pulmonary disease: a systematic review. Chron Respir Dis 2010;7:9-17.

53 Pitkin $A D$, Roberts $C M$, Wedzicha JA. Arterialised earlobe blood gas analysis: an underused technique. Thorax 1994;49:364-6.

54 Maas $\mathrm{AH}$, van Heijst HA. A comparison of the $\mathrm{pH}$ of arterial blood with arterialised blood from the ear-lobe with Astrup's micro glasselectrode. Clin Chim Acta 1961;6:31-3.

55 Cox M, Kemp R, Anwar S, et al. Non-invasive monitoring of $\mathrm{CO} 2$ levels in patients using NIV for AECOPD. Thorax 2006;61:363-4.

56 Gancel PE, Roupie E, Guittet L, et al. Accuracy of a transcutaneous carbon dioxide pressure monitoring device in emergency room patients with acute respiratory failure. Intensive Care Med 2011;37:348-51.

57 Nicolini A, Ferrari MB. Evaluation of a transcutaneous carbon dioxide monitor in patients with acute respiratory failure. Ann Thorac Med 2011;6:217-20.

58 van Oppen JD, Daniel PS, Sovani MP. What is the potential role of transcutaneous carbon dioxide in guiding acute noninvasive ventilation? Respir Care 2015:60:484-91.

59 Jeffrey AA, Warren PM, Flenley DC. Acute hypercapnic respiratory failure in patients with chronic obstructive lung disease: risk factors and use of guidelines for management. Thorax 1992;47:34-40.

60 Kaul S, Pearson M, Coutts I, et al. Non-invasive ventilation (NIV) in the clinical management of acute COPD in 233 UK hospitals: results from the RCP/BTS 2003 National COPD Audit. COPD 2009;6:171-6.

61 Austin MA, Wills KE, Blizzard L, et al. Effect of high flow oxygen on mortality in chronic obstructive pulmonary disease patients in prehospital setting: randomised controlled trial. BMJ 2010;341:c5462.

62 O'Driscoll BR, Howard LS, Davison AG, et al. BTS guideline for emergency oxygen use in adult patients. Thorax 2008;63(Suppl_6):vi1-68.

63 Padkin AJ, Kinnear WJM. Supplemental oxygen and nasal intermittent positive pressure ventilation. Eur Respir J 1996;9:834-6.

64 Kaul S. The effect of entrainment site and inspiratory pressure on the delivery of oxygen therapy during non-invasive mechanical ventilation (NIMV) in acute COPD patients. Eur Respir Rev 2006;15:190-1.

65 Tuggey JM, Delmastro M, Elliott MW. The effect of mouth leak and humidification during nasal non-invasive ventilation. Respir Med 2007;101:1874-9.

66 Oto J, Imanaka H, Nishimura M. Clinical factors affecting inspired gas humidification and oral dryness during noninvasive ventilation. J Crit Care 2011;26:535.e9-15.

67 Oto J, Nakataki E, Okuda N, et al. Hygrometric properties of inspired gas and oral dryness in patients with acute respiratory failure during noninvasive ventilation. Respir Care 2014;59:39-45.

68 Kaul S. Improving the patient-ventilator interaction during non-invasive ventilation in patients with COPD [PhD]. University of London, 2010. 
69 Mukhopadhyay A, Dela PE, Wadden B, et al. Effects of inhalational bronchodilator treatment during noninvasive ventilation in severe chronic obstructive pulmonary disease exacerbations. J Crit Care 2009;24:474.e1-5.

70 Abdelrahim ME, Plant P, Chrystyn H. In-vitro characterisation of the nebulised dose during non-invasive ventilation. J Pharm Pharmacol 2010;62:966-72.

71 Devlin JW, Nava S, Fong JJ, et al. Survey of sedation practices during noninvasive positive-pressure ventilation to treat acute respiratory failure. Crit Care Med 2007:35:2298-302.

72 Clouzeau B, Bui HN, Vargas F, et al. Target-controlled infusion of propofol for sedation in patients with non-invasive ventilation failure due to low tolerance: a preliminary study. Intensive Care Med 2010;36:1675-80.

73 Akada S, Takeda S, Yoshida Y, et al. The efficacy of dexmedetomidine in patients with noninvasive ventilation: a preliminary study. Anesth Analg 2008; 107:167-70.

74 Constantin JM, Schneider E, Cayot-Constantin S, et al. Remifentanil-based sedation to treat noninvasive ventilation failure: a preliminary study. Intensive Care Med 2007;33:82-7.

75 Rocco M, Conti G, Alessandri E, et al. Rescue treatment for noninvasive ventilation failure due to interface intolerance with remifentanil analgosedation: a pilot study. Intensive Care Med 2010;36:2060-5.

76 Senoglu N, Oksuz H, Dogan Z, et al. Sedation during noninvasive mechanical ventilation with dexmedetomidine or midazolam: a randomized, double-blind, prospective study. Curr Ther Res Clin Exp 2010;71:141-53.

77 Devlin JW, Al-Qadheeb NS, Chi A, et al. Efficacy and safety of early dexmedetomidine during noninvasive ventilation for patients with acute respiratory failure: a randomized, double-blind, placebo-controlled pilot study. Chest 2014;145:1204-12.

78 Longrois D, Conti G, Mantz J, et al. Sedation in non-invasive ventilation: do we know what to do (and why)? Multidiscip Respir Med 2014;9:56.

79 Safe sedation practice for healthcare procedures_-standards and guidance. 2013. http://www.aomrc.org.uk/doc_details/9737-safe-sedation-practice-for-healthcareprocedures-standards-and-guidance

80 Nava S, Navalesi P, Gregoretti C. Interfaces and humidification for noninvasive mechanical ventilation. Respir Care 2009;54:71-84.

81 Carron M, Freo U, BaHammam AS, et al. Complications of non-invasive ventilation techniques: a comprehensive qualitative review of randomized trials. $\mathrm{Br} J$ Anaesth 2013;110:896-914.

82 Fukushima K, Marut K, Kiyofuji C, et al. [Evaluation of the incidence of pneumothorax and background of patients with pneumothorax during noninvasive positive pressure ventilation]. Nihon Kokyuki Gakkai Zasshi 2008:46:870-4.

83 Chatwin M, Ross E, Hart N, et al. Cough augmentation with mechanical insufflation/exsufflation in patients with neuromuscular weakness. Eur Respir J 2003:21:502-8.

84 Chatwin M, Simonds AK. The addition of mechanical insufflation/exsufflation shortens airway-clearance sessions in neuromuscular patients with chest infection. Respir Care 2009;54:1473-9.

85 Sivasothy P, Brown L, Smith IE, et al. Effect of manually assisted cough and mechanical insufflation on cough flow of normal subjects, patients with chronic obstructive pulmonary disease (COPD), and patients with respiratory muscle weakness. Thorax 2001;56:438-44.

86 Goncalves MR, Honrado T, Winck JC, et al. Effects of mechanical insufflation-exsufflation in preventing respiratory failure after extubation: a randomized controlled trial. Crit Care 2012;16:R48.

87 Bott J, Blumenthal S, Buxton M, et al. Guidelines for the physiotherapy management of the adult, medical, spontaneously breathing patient. Thorax 2009;64(Suppl 1):i1-51.

88 Wright CD. Minitracheostomy. Clin Chest Med 2003;24:431-5

89 Core topics in mechanical ventilation. Cambridge University Press, 2008.

90 Principles and practice of mechanical ventilation. McGraw Hill, 2012.

91 Gursel G, Aydogdu M, Tasyurek S, et al. Factors associated with noninvasive ventilation response in the first day of therapy in patients with hypercapnic respiratory failure. Ann Thorac Med 2012;7:92-7.

92 Murphy PB, Davidson C, Hind MD, et al. Volume targeted versus pressure support non-invasive ventilation in patients with super obesity and chronic respiratory failure: a randomised controlled trial. Thorax 2012;67:727-34.

93 Roche Campo F, Drouot X, Thille AW, et al. Poor sleep quality is associated with late noninvasive ventilation failure in patients with acute hypercapnic respiratory failure. Crit Care Med 2010;38:477-85.

94 Levine $\mathrm{S}$, Nguyen T, Taylor N, et al. Rapid disuse atrophy of diaphragm fibers in mechanically ventilated humans. N Engl J Med 2008;358:1327-35.

95 Putensen $\mathrm{C}$, Zech $\mathrm{S}$, Wrigge $\mathrm{H}$, et al. Long-term effects of spontaneous breathing during ventilatory support in patients with acute lung injury. Am J Respir Crit Care Med 2001;164:43-9.

96 Ward NS, Dushay KM. Clinical concise review: mechanical ventilation of patients with chronic obstructive pulmonary disease. Crit Care Med 2008;36:1614-19.
97 Gladwin MT, Pierson DJ. Mechanical ventilation of the patient with severe chronic obstructive pulmonary disease. Intensive Care Med 1998;24:898-910.

98 Fougeres E, Teboul JL, Richard C, et al. Hemodynamic impact of a positive end-expiratory pressure setting in acute respiratory distress syndrome: importance of the volume status. Crit Care Med 2010;38:802-7.

99 Tuxen DV, Lane $S$. The effects of ventilatory pattern on hyperinflation, airway pressures, and circulation in mechanical ventilation of patients with severe air-flow obstruction. Am Rev Respir Dis 1987:136:872-9.

100 Leatherman JW, McArthur C, Shapiro RS. Effect of prolongation of expiratory time on dynamic hyperinflation in mechanically ventilated patients with severe asthma. Crit Care Med 2004;32:1542-5.

101 Ranieri VM, Suter PM, Tortorella C, et al. Effect of mechanical ventilation on inflammatory mediators in patients with acute respiratory distress syndrome: a randomized controlled trial. JAMA 1999;282:54-61.

102 The Acute Respiratory Distress Syndrome Network. Ventilation with lower tidal volumes as compared with traditional tidal volumes for acute lung injury and the acute respiratory distress syndrome. The Acute Respiratory Distress Syndrome Network. N Engl J Med 2000;342:1301-8.

103 Kregenow DA, Rubenfeld GD, Hudson LD, et al. Hypercapnic acidosis and mortality in acute lung injury. Crit Care Med 2006;34:1-7.

104 Amato MB, Barbas CS, Medeiros DM, et al. Effect of a protective-ventilation strategy on mortality in the acute respiratory distress syndrome. $N$ Engl I Med 1998;338:347-54.

105 Adnet F, Plaisance P, Borron SW, et al. Prolonged severe hypercapnia complicating near fatal asthma in a 35-year-old woman. Intensive Care Med 1998;24:1335-8.

106 Swenson ER. Carbonic anhydrase inhibitors and ventilation: a complex interplay of stimulation and suppression. Eur Respir J 1998;12:1242-7.

107 Jones PW, Greenstone M. Carbonic anhydrase inhibitors for hypercapnic ventilatory failure in chronic obstructive pulmonary disease. Cochrane Database Syst Rev 2001;(1):CD002881.

108 Aerts JG, van den Berg B, Bogaard JM. Controlled expiration in mechanically-ventilated patients with chronic obstructive pulmonary disease (COPD). Eur Respir J 1997;10:550-6.

109 Kondili E, Alexopoulou C, Prinianakis G, et al. Pattern of lung emptying and expiratory resistance in mechanically ventilated patients with chronic obstructive pulmonary disease. Intensive Care Med 2004;30:1311-18.

110 Brochard L. Intrinsic (or auto-) PEEP during controlled mechanical ventilation. Intensive Care Med 2002;28:1376-8.

111 Kress JP, O'Connor MF, Schmidt GA. Clinical examination reliably detects intrinsic positive end-expiratory pressure in critically ill, mechanically ventilated patients. Am J Respir Crit Care Med 1999;159:290-4.

112 Blanch L, Bernabe F, Lucangelo U. Measurement of air trapping, intrinsic positive end-expiratory pressure, and dynamic hyperinflation in mechanically ventilated patients. Respir Care 2005;50:110-23.

113 Rossi A, Ganassini A, Polese G, et al. Pulmonary hyperinflation and ventilator-dependent patients. Eur Respir J 1997;10:1663-74.

114 Ranieri VM, Giuliani R, Cinnella G, et al. Physiologic effects of positive end-expiratory pressure in patients with chronic obstructive pulmonary disease during acute ventilatory failure and controlled mechanical ventilation. Am Rev Respir Dis 1993;147:5-13.

115 Rossi A, Santos C, Roca J, et al. Effects of PEEP on VA/Q mismatching in ventilated patients with chronic airflow obstruction. Am J Respir Crit Care Med 1994;149:1077-84.

116 Guerin C, LeMasson S, de Varax R, et al. Small airway closure and positive end-expiratory pressure in mechanically ventilated patients with chronic obstructive pulmonary disease. Am J Respir Crit Care Med 1997;155:1949-56.

117 Caramez MP, Borges JB, Tucci MR, et al. Paradoxical responses to positive end-expiratory pressure in patients with airway obstruction during controlled ventilation. Crit Care Med 2005:33:1519-28.

118 MacIntyre NR, Cheng KC, McConnell R. Applied PEEP during pressure support reduces the inspiratory threshold load of intrinsic PEEP. Chest 1997;111:188-93.

119 Nava S, Bruschi C, Rubini F, et al. Respiratory response and inspiratory effort during pressure support ventilation in COPD patients. Intensive Care Med 1995;21:871-9.

120 Guerin C, Milic-Emili J, Fournier G. Effect of PEEP on work of breathing in mechanically ventilated COPD patients. Intensive Care Med 2000;26:1207-14.

121 Spina SP, Ensom MH. Clinical pharmacokinetic monitoring of midazolam in critically ill patients. Pharmacotherapy 2007;27:389-98.

122 Shafer A. Complications of sedation with midazolam in the intensive care unit and a comparison with other sedative regimens. Crit Care Med 1998;26: 947-56.

123 Barr J, Fraser GL, Puntillo K, et al. Clinical practice guidelines for the management of pain, agitation, and delirium in adult patients in the intensive care unit. Crit Care Med 2013:41:263-306.

124 Kress JP, Pohlman AS, O'Connor MF, et al. Daily interruption of sedative infusions in critically ill patients undergoing mechanical ventilation. $N$ Engl I Med 2000;342:1471-7. 
125 Girard TD, Kress JP, Fuchs BD, et al. Efficacy and safety of a paired sedation and ventilator weaning protocol for mechanically ventilated patients in intensive care (Awakening and Breathing Controlled trial): a randomised controlled trial. Lancet 2008:371:126-34

126 Brook AD, Ahrens TS, Schaiff $R$, et al. Effect of a nursing-implemented sedation protocol on the duration of mechanical ventilation. Crit Care Med 1999;27:2609-15

127 Arias-Rivera S, Sanchez-Sanchez Mdel M, Santos-Diaz R, et al. Effect of a nursing-implemented sedation protocol on weaning outcome. Crit Care Med 2008;36:2054-60

128 Patel SB, Kress JP. Sedation and analgesia in the mechanically ventilated patient. Am J Respir Crit Care Med 2012;185:486-97.

129 Hughes CG, Girard TD, Pandharipande PP. Daily sedation interruption versus targeted light sedation strategies in ICU patients. Crit Care Med 2013;41(9 Suppl 1):S39-45.

130 Augustes R, Ho KM. Meta-analysis of randomised controlled trials on daily sedation interruption for critically ill adult patients. Anaesth Intensive Care 2011;39:401-9.

131 Mehta S, Burry L, Cook D, et al. Daily sedation interruption in mechanically ventilated critically ill patients cared for with a sedation protocol: a randomized controlled trial. JAMA 2012;308:1985-92.

132 Epstein SK. How often does patient-ventilator asynchrony occur and what are the consequences? Respir Care 2011;56:25-38

133 de Wit M, Miller KB, Green DA, et al. Ineffective triggering predicts increased duration of mechanical ventilation. Crit Care Med 2009;37: 2740-5.

134 Chao DC, Scheinhorn DJ, Stearn-Hassenpflug M. Patient-ventilator trigger asynchrony in prolonged mechanical ventilation. Chest 1997;112:1592-9.

135 Colombo D, Cammarota G, Alemani M, et al. Efficacy of ventilator waveforms observation in detecting patient-ventilator asynchrony. Crit Care Med 2011;39:2452-7.

136 Thille AW, Lyazidi A, Richard JC, et al. A bench study of intensive-care-unit ventilators: new versus old and turbine-based versus compressed gas-based ventilators. Intensive Care Med 2009;35:1368-76.

137 Richard JC, Carlucci A, Breton L, et al. Bench testing of pressure support ventilation with three different generations of ventilators. Intensive Care Med 2002;28:1049-57

138 Maclntyre NR, McConnell R, Cheng KC, et al. Patient-ventilator flow dyssynchrony: flow-limited versus pressure-limited breaths. Crit Care Med 1997;25:1671-7.

139 Thille AW, Rodriguez P, Cabello B, et al. Patient-ventilator asynchrony during assisted mechanical ventilation. Intensive Care Med 2006;32:1515-22.

140 Passam F, Hoing S, Prinianakis G, et al. Effect of different levels of pressure support and proportional assist ventilation on breathing pattern, work of breathing and gas exchange in mechanically ventilated hypercapnic COPD patients with acute respiratory failure. Respiration 2003;70:355-61.

141 Bosma K, Ferreyra G, Ambrogio C, et al. Patient-ventilator interaction and sleep in mechanically ventilated patients: pressure support versus proportional assist ventilation. Crit Care Med 2007:35:1048-54.

142 Costa R, Spinazzola G, Cipriani F, et al. A physiologic comparison of proportional assist ventilation with load-adjustable gain factors (PAV+) versus pressure support ventilation (PSV). Intensive Care Med 2011;37:1494-500.

143 Xirouchaki N, Kondili E, Vaporidi K, et al. Proportional assist ventilation with load-adjustable gain factors in critically ill patients: comparison with pressure support. Intensive Care Med 2008;34:2026-34.

144 Spahija J, de Marchie M, Albert M, et al. Patient-ventilator interaction during pressure support ventilation and neurally adjusted ventilatory assist. Crit Care Med 2010;38:518-26.

145 Piquilloud L, Vignaux L, Bialais E, et al. Neurally adjusted ventilatory assist improves patient-ventilator interaction. Intensive Care Med 2011;37: 263-71.

146 Scales DC, Thiruchelvam D, Kiss A, et al. The effect of tracheostomy timing during critical illness on long-term survival. Crit Care Med 2008;36: 2547-57.

147 Rumbak MJ, Newton M, Truncale T, et al. A prospective, randomized, study comparing early percutaneous dilational tracheotomy to prolonged translaryngeal intubation (delayed tracheotomy) in critically ill medical patients. Crit Care Med 2004;32:1689-94

148 Griffiths J, Barber VS, Morgan L, et al. Systematic review and meta-analysis of studies of the timing of tracheostomy in adult patients undergoing artificial ventilation. BMJ 2005:330:1243.

149 Krishnan K, Elliot SC, Mallick A. The current practice of tracheostomy in the United Kingdom: a postal survey. Anaesthesia 2005;60:360-4.

150 Terragni PP, Antonelli M, Fumagalli R, et al. Early vs late tracheotomy for prevention of pneumonia in mechanically ventilated adult ICU patients: a randomized controlled trial. JAMA 2010;303:1483-9.

151 Young D, Harrison DA, Cuthbertson BH, et al. Effect of early vs late tracheostomy placement on survival in patients receiving mechanical ventilation: the TracMan randomized trial. JAMA 2013;309:2121-9.
152 Wang $F$, Wu Y, Bo L, et al. The timing of tracheotomy in critically ill patients undergoing mechanical ventilation: a systematic review and meta-analysis of randomized controlled trials. Chest 2011;140:1456-65.

153 Dempsey GA, Grant CA, Jones TM. Percutaneous tracheostomy: a 6 yr prospective evaluation of the single tapered dilator technique. $\mathrm{Br} J$ Anaesth 2010;105:782-8.

154 Silvester W, Goldsmith D, Uchino S, et al. Percutaneous versus surgical tracheostomy: a randomized controlled study with long-term follow-up. Crit Care Med 2006;34:2145-52.

155 Cook TM, Woodall N, Harper J, et al. Major complications of airway management in the UK: results of the Fourth National Audit Project of the Royal College of Anaesthetists and the Difficult Airway Society. Part 2: intensive care and emergency departments. Br J Anaesth 2011;106:632-42.

156 Durrington $\mathrm{HJ}$, Flubacher M, Ramsay CF, et al. Initial oxygen management in patients with an exacerbation of chronic obstructive pulmonary disease. QJM 2005;98:499-504.

157 Nava S, Navalesi P, Conti G. Time of non-invasive ventilation. Intensive Care Med 2006;32:361-70.

158 Conti G, Antonelli M, Navalesi P, et al. Noninvasive vs. conventional mechanical ventilation in patients with chronic obstructive pulmonary disease after failure of medical treatment in the ward: a randomized trial. Intensive Care Med 2002;28:1701-7.

159 Wood KA, Lewis L, Von Harz B, et al. The use of Noninvasive positive pressure ventilation in the Emergency Department. Chest 1998;113:1339-46.

160 Chandra D, Stamm JA, Taylor B, et al. Outcomes of noninvasive ventilation for acute exacerbations of chronic obstructive pulmonary disease in the United States, 1998-2008. Am J Respir Crit Care Med 2012;185:152-9.

161 Elliott MW, Nava S. Noninvasive ventilation for acute exacerbations of chronic obstructive pulmonary disease: "Don't think twice, it's alright!" Am J Respir Crit Care Med 2012;185:121-3.

162 Collaborative Research Group of Noninvasive Mechanical Ventilation for Chronic Obstructive Pulmonary Disease. Early use of non-invasive positive pressure ventilation for acute exacerbations of chronic obstructive pulmonary disease: a multicentre randomized controlled trial. Chin Med J 2005;118:2034-40.

163 Plant PK, Owen JL, Elliott MW. Early use of non-invasive ventilation for acute exacerbations of chronic obstructive pulmonary disease on general respiratory wards: a multicentre randomised controlled trial. Lancet 2000;355:1931-5.

164 Pierson DJ. Indications for mechanical ventilation in adults with acute respiratory failure. Respir Care 2002;47:249-62.

165 Price LC, Lowe D, Hosker HS, et al. UK National COPD Audit 2003: impact of hospital resources and organisation of care on patient outcome following admission for acute COPD exacerbation. Thorax 2006;61:837-42.

166 Keenan SP, Mehta S. Noninvasive ventilation for patients presenting with acute respiratory failure: the randomized controlled trials. Respir Care 2009;54:116-26.

167 Ucgun I, Metintas M, Moral H, et al. Predictors of hospital outcome and intubation in COPD patients admitted to the respiratory ICU for acute hypercapnic respiratory failure. Respir Med 2006;100:66-74.

168 Scala R, Naldi M, Archinucci I, et al. Noninvasive positive pressure ventilation in patients with acute exacerbations of COPD and varying levels of consciousness. Chest 2005:128:1657-66.

169 Nava S, Grassi M, Fanfulla F, et al. Non-invasive ventilation in elderly patients with acute hypercapnic respiratory failure: a randomised controlled trial. Age Ageing 2011:40:444-50.

170 Riario-Sforza GG, Scarpazza P, Incorvaia C, et al. Role of noninvasive ventilation in elderly patients with hypercapnic respiratory failure. Clin Ter 2012;163:e47-52.

171 Miller D, Fraser K, Murray l, et al. Predicting survival following non-invasive ventilation for hypercapnic exacerbations of chronic obstructive pulmonary disease. Int J Clin Pract 2012;66:434-7.

172 Plant PK, Owen JL, Elliott MW. Non-invasive ventilation in acute exacerbations of chronic obstructive pulmonary disease: long term survival and predictors of in-hospital outcome. Thorax 2001;56:708-12.

173 Confalonieri M, Garuti G, Cattaruzza MS, et al. A chart of failure risk for noninvasive ventilation in patients with COPD exacerbation. Eur Respir J 2005:25:348-55.

174 Keenan SP. Noninvasive positive-pressure ventilation for postextubation respiratory distress: a randomized controlled trial. JAMA 2002;287:3238-44.

175 Khilnani GC, Banga A. Noninvasive ventilation in patients with chronic obstructive airway disease. Int J Chron Obstruct Pulmon Dis 2008;3:351-7.

176 Lightowler JV, Wedzicha JA, Elliott MW, et al. Non-invasive positive pressure ventilation to treat respiratory failure resulting from exacerbations of chronic obstructive pulmonary disease: cochrane systematic review and meta-analysis. BMJ 2003;326:185-9.

177 Moretti M, Cilione C, Tampieri A, et al. Incidence and causes of non-invasive mechanical ventilation failure after initial success. Thorax 2000;55:819-25.

178 Windisch W, Storre JH. Target volume settings for home mechanical ventilation: great progress or just a gadget? Thorax 2012;67:663-5.

179 Kramer N, Meyer TJ, Meharg J, et al. Randomized, prospective trial of noninvasive positive pressure ventilation in acute respiratory failure. Am J Respir Crit Care Med 1995;151:1799-806 
180 Phua J, Kong K, Lee KH, et al. Noninvasive ventilation in hypercapnic acute respiratory failure due to chronic obstructive pulmonary disease vs. other conditions: effectiveness and predictors of failure. Intensive Care Med 2005:31:533-9.

181 Esteban A, Frutos-Vivar F, Ferguson ND, et al. Noninvasive positive-pressure ventilation for respiratory failure after extubation. $N$ Engl J Med 2004;350:2452-60.

182 Wildman MJ, Sanderson C, Groves J, et al. Implications of prognostic pessimism in patients with chronic obstructive pulmonary disease (COPD) or asthma admitted to intensive care in the UK within the COPD and asthma outcome study (CAOS): multicentre observational cohort study. BMJ 2007;335:1132.

183 Sumner K, Yadegafar G. The utility and futility of non-invasive ventilation in non-designated areas: can critical care outreach nurses influence practice? Intensive Crit Care Nurs 2011;27:211-17.

184 Celli BR, Cote CG, Marin JM, et al. The body-mass index, airflow obstruction, dyspnea, and exercise capacity index in chronic obstructive pulmonary disease. N Engl J Med 2004;350:1005-12.

185 Steer J, Gibson J, Bourke SC. The DECAF Score: predicting hospital mortality in exacerbations of chronic obstructive pulmonary disease. Thorax 2012;67:970-6.

186 Liu H, Zhang T, Ye J. Determinants of prolonged mechanical ventilation in patients with chronic obstructive pulmonary diseases and acute hypercapnic respiratory failure. Eur J Intern Med 2007;18:542-7.

187 Goel A, Pinckney RG, Littenberg B. APACHE II predicts long-term survival in COPD patients admitted to a general medical ward. J Gen Intern Med 2003;18: 824-30.

188 Wildman MJ, Harrison DA, Welch CA, et al. A new measure of acute physiologica derangement for patients with exacerbations of obstructive airways disease: the COPD and Asthma Physiology Score. Respir Med 2007;101:1994-2002.

189 Confalonieri M, Parigi P, Scartabellati A, et al. Noninvasive mechanical ventilation improves the immediate and long-term outcome of COPD patients with acute respiratory failure. Eur Respir J 1996;9:422-30.

190 Suissa S, Dell'Aniello S, Ernst P. Long-term natural history of chronic obstructive pulmonary disease: severe exacerbations and mortality. Thorax 2012;67:957-63.

191 Chung LP, Winship P, Phung S, et al. Five-year outcome in COPD patients after their first episode of acute exacerbation treated with non-invasive ventilation. Respirology 2010;15:1084-91.

192 Chu CM, Chan VL, Lin AW, et al. Readmission rates and life threatening events in COPD survivors treated with non-invasive ventilation for acute hypercapnic respiratory failure. Thorax 2004;59:1020-5.

193 Wildman MJ, Sanderson CF, Groves J, et al. Survival and quality of life for patients with COPD or asthma admitted to intensive care in a UK multicentre cohort: the COPD and Asthma Outcome Study (CAOS). Thorax 2009;64:128-32.

194 Brandao DC, Lima VM, Filho VG, et al. Reversal of bronchial obstruction with bi-level positive airway pressure and nebulization in patients with acute asthma. J Asthma 2009:46:356-61.

195 Holley MT, Morrissey TK, Seaberg DC, et al. Ethical dilemmas in a randomized trial of asthma treatment: can Bayesian statistical analysis explain the results? Acad Emerg Med 2001:8:1128-35.

196 Gupta D, Nath A, Agarwal R, et al. A prospective randomized controlled trial on the efficacy of noninvasive ventilation in severe acute asthma. Respir Care 2010;55:536-43.

197 Soma T, Hino M, Kida K, et al. A prospective and randomized study for improvement of acute asthma by non-invasive positive pressure ventilation (NPPV) Intern Med 2008;47:493-501.

198 Soroksky A, Stav D, Shpirer I. A pilot prospective, randomized, placebo-controlled trial of bilevel positive airway pressure in acute asthmatic attack. Chest 2003;123:1018-25.

199 Lim WJ, Mohammed Akram R, Carson KV, et al. Non-invasive positive pressure ventilation for treatment of respiratory failure due to severe acute exacerbations of asthma. Cochrane Database Syst Rev 2012;12:CD004360.

200 Meduri GU, Cook TR, Turner RE, et al. Noninvasive positive pressure ventilation in status asthmaticus. Chest 1996;110:767-74.

201 Wilson DH, Tucker G, Frith P, et al. Trends in hospital admissions and mortality from asthma and chronic obstructive pulmonary disease in Australia, 1993-2003. Med J Aust 2007;186:408-11.

202 Pasteur MC, Bilton D, Hill AT. British Thoracic Society guideline for non-CF bronchiectasis. Thorax 2010;65(Suppl 1):i1-58.

203 Phua J, Ang YL, See KC, et al. Noninvasive and invasive ventilation in acute respiratory failure associated with bronchiectasis. Intensive Care Med 2010;36:638-47.

204 Efrati O, Bylin I, Segal E, et al. Outcome of patients with cystic fibrosis admitted to the intensive care unit: is invasive mechanical ventilation a risk factor for death in patients waiting lung transplantation? Heart Lung 2010;39:153-9.

205 Slieker MG, van Gestel JP, Heijerman HG, et al. Outcome of assisted ventilation for acute respiratory failure in cystic fibrosis. Intensive Care Med 2006;32: 754-8.

206 Sheikh HS, Tiangco ND, Harrell C, et al. Severe hypercapnia in critically ill adult cystic fibrosis patients. J Clin Med Res 2011;3:209-12.
207 Vedam H, Moriarty C, Torzillo PJ, et al. Improved outcomes of patients with cystic fibrosis admitted to the intensive care unit. J Cyst Fibros 2004;3:8-14.

208 Hodson ME, Madden BP, Steven MH, et al. Non-invasive mechanical ventilation for cystic fibrosis patients-a potential bridge to transplantation. Eur Respir J 1991;4:524-7

209 Madden BP, Kariyawasam H, Siddiqi AJ, et al. Noninvasive ventilation in cystic fibrosis patients with acute or chronic respiratory failure. Eur Respir J 2002;19:310-13.

210 Texereau J, Jamal D, Choukroun G, et al. Determinants of mortality for adults with cystic fibrosis admitted in Intensive Care Unit: a multicenter study. Respir Res 2006;7:14.

211 Jones A, Bilton D, Evans TW, et al. Predictors of outcome in patients with cystic fibrosis requiring endotracheal intubation. Respirology 2013;18: 630-6.

212 NICE clinical guidance [CG105]. Motor neurone disease: the use of non-invasive ventilation in the management of motor neurone disease. 2010. http://www.nice. org.uk/guidance/cg105

213 Bourke SC, Tomlinson M, Williams TL, et al. Effects of non-invasive ventilation on survival and quality of life in patients with amyotrophic lateral sclerosis: a randomised controlled trial. Lancet Neurol 2006;5:140-7.

214 Chio A, Calvo A, Ghiglione P, et al. Tracheostomy in amyotrophic lateral sclerosis: a 10-year population-based study in Italy. J Neurol Neurosurg Psychiatr 2010;81:1141-3.

215 Simonds AK. NIV in neuromuscular disease. In: Muir J-F, Ambrosino N, Simonds A eds. Noninvasive ventilation. 2nd edn. European Respiratory Society Monograph, 2008:224-39.

216 Bradley MD, Orrell RW, Clarke J, et al. Outcome of ventilatory support for acute respiratory failure in motor neurone disease. J Neurol Neurosurg Psychiatr 2002;72:752-6

217 Sancho J, Servera E, Diaz JL, et al. Home tracheotomy mechanical ventilation in patients with amyotrophic lateral sclerosis: causes, complications and 1-year survival. Thorax 2011:66:948-52.

218 Chadwick R, Nadig V, Oscroft NS, et al. Weaning from prolonged invasive ventilation in motor neuron disease: analysis of outcomes and survival. J Neurol Neurosurg Psychiatry 2011;82:643-5.

219 Shneerson JM. Who will benefit from tracheostomy ventilation in motor neuron disease? Thorax 2011;66:932-3.

220 Nowbar S, Burkart KM, Gonzales R, et al. Obesity-associated hypoventilation in hospitalized patients: prevalence, effects, and outcome. Am J Med 2004;116:1-7

221 Perez de Llano LA, Golpe R, Ortiz Piquer M, et al. Short-term and long-term effects of nasal intermittent positive pressure ventilation in patients with obesity-hypoventilation syndrome. Chest 2005; 128:587-94.

222 Resta 0, Foschino-Barbaro MP, Bonfitto $\mathrm{P}$, et al. Prevalence and mechanisms of diurnal hypercapnia in a sample of morbidly obese subjects with obstructive sleep apnoea. Respir Med 2000;94:240-6.

223 Wagner DP. Economics of prolonged mechanical ventilation. Am Rev Respir Dis 1989;140(2 Pt 2):S14-18.

224 Epstein $C D$, Peerless JR. Weaning readiness and fluid balance in older critically ill surgical patients. Am J Crit Care 2006;15:54-64.

225 Yang KL, Tobin MJ. A prospective study of indexes predicting the outcome of trials of weaning from mechanical ventilation. N Engl J Med 1991;324:1445-50.

226 Laghi F, Tobin MJ. Disorders of the respiratory muscles. Am J Respir Crit Care Med 2003; 168:10-48.

227 Vincent JL, Sakr Y, Sprung CL, et al. Sepsis in European intensive care units: results of the SOAP study. Crit Care Med 2006;34:344-53.

228 Wiedemann HP, Wheeler AP, Bernard GR, et al. Comparison of two fluid-management strategies in acute lung injury. $N$ Engl J Med 2006:354:2564-75.

229 Payen D, de Pont AC, Sakr Y, et al. A positive fluid balance is associated with a worse outcome in patients with acute renal failure. Crit Care 2008;12:R74.

230 Upadya A, Tilluckdharry L, Muralidharan V, et al. Fluid balance and weaning outcomes. Intensive Care Med 2005:31:1643-7.

231 Mekontso Dessap A, Roche-Campo F, Kouatchet A, et al. Natriuretic peptide-driven fluid management during ventilator weaning: a randomized controlled trial. Am J Respir Crit Care Med 2012;186:1256-63.

232 Brochard L, Rauss A, Benito S, et al. Comparison of three methods of gradual withdrawal from ventilatory support during weaning from mechanical ventilation. Am J Respir Crit Care Med 1994;150:896-903.

233 Esteban A, Frutos F, Tobin MJ, et al. A comparison of four methods of weaning patients from mechanical ventilation. Spanish Lung Failure Collaborative Group. N Engl J Med 1995;332:345-50.

234 Vitacca M, Vianello A, Colombo D, et al. Comparison of two methods for weaning patients with chronic obstructive pulmonary disease requiring mechanical ventilation for more than 15 days. Am J Respir Crit Care Med 2001;164:225-30.

235 Frutos-Vivar F, Ferguson ND, Esteban A, et al. Risk factors for extubation failure in patients following a successful spontaneous breathing trial. Chest 2006;130:1664-71. 
236 Esteban A, Alia I, Gordo F, et al. Extubation outcome after spontaneous breathing trials with T-tube or pressure support ventilation. The Spanish Lung Failure Collaborative Group. Am J Respir Crit Care Med 1997;156(2 Pt 1): $459-65$.

237 Perren A, Domenighetti G, Mauri S, et al. Protocol-directed weaning from mechanical ventilation: clinical outcome in patients randomized for a 30-min or 120-min trial with pressure support ventilation. Intensive Care Med 2002:28:1058-63.

238 Ely EW, Baker AM, Dunagan DP, et al. Effect on the duration of mechanical ventilation of identifying patients capable of breathing spontaneously. $N$ Engl J Med 1996:335:1864-9.

239 Vallverdu I, Calaf N, Subirana M, et al. Clinical characteristics, respiratory functional parameters, and outcome of a two-hour T-piece trial in patients weaning from mechanical ventilation. Am J Respir Crit Care Med 1998:158:1855-62.

240 Epstein SK. Decision to extubate. Intensive Care Med 2002;28:535-46.

241 Boles JM, Bion J, Connors A, et al. Weaning from mechanical ventilation. Eur Respir J 2007;29:1033-56.

242 Namen AM, Ely EW, Tatter SB, et al. Predictors of successful extubation in neurosurgical patients. Am J Respir Crit Care Med 2001;163(3 Pt 1):658-64.

243 Salam A, Tilluckdharry L, Amoateng-Adjepong Y, et al. Neurologic status, cough, secretions and extubation outcomes. Intensive Care Med 2004;30:1334-9.

244 Rothaar RC, Epstein SK. Extubation failure: magnitude of the problem, impact on outcomes, and prevention. Curr Opin Crit Care 2003;9:59-66.

245 Blackwood B, Alderdice F, Burns K, et al. Use of weaning protocols for reducing duration of mechanical ventilation in critically ill adult patients: Cochrane systematic review and meta-analysis. BMJ 2011;342:c7237.

246 Piotto RF, Maia LN, Machado MN, et al. Effects of the use of mechanical ventilation weaning protocol in the Coronary Care Unit: randomized study. Rev Bras Cir Cardiovasc 2011;26:213-21.

247 Lellouche $\mathrm{F}$, Mancebo J, Jolliet $\mathrm{P}$, et al. A multicenter randomized trial of computer-driven protocolized weaning from mechanical ventilation. Am J Respir Crit Care Med 2006:174:894-900.

248 Rose L, Presneill JJ, Johnston L, et al. A randomised, controlled trial of conventional versus automated weaning from mechanical ventilation using SmartCare/PS. Intensive Care Med 2008;34:1788-95.

249 Kilger $\mathrm{E}$, Briegel J, Haller $\mathrm{M}$, et al. [Noninvasive ventilation after lung transplantation]. Med Klin (Munich) 1995;90(1 Suppl 1):26-8.

250 Ferrer M, Esquinas A, Arancibia F, et al. Noninvasive ventilation during persistent weaning failure: a randomized controlled trial.[see comment]. Am J Respir Crit Care Med 2003;168:70-6

251 Girault C, Bubenheim M, Abroug F, et al. Noninvasive ventilation and weaning in patients with chronic hypercapnic respiratory failure: a randomized multicenter trial. Am J Respir Crit Care Med 2011;184:672-9.

252 Burns KE, Meade MO, Premji A, et al. Noninvasive ventilation as a weaning strategy for mechanical ventilation in adults with respiratory failure: a Cochrane systematic review. CMAJ 2014;186:E112-22.

253 Ferrer M, Valencia M, Nicolas JM, et al. Early noninvasive ventilation averts extubation failure in patients at risk: a randomized trial. Am J Respir Crit Care Med 2006;173:164-70.

254 El-Solh AA, Aquilina A, Pineda L, et al. Noninvasive ventilation for prevention of post-extubation respiratory failure in obese patients. Eur Respir J 2006:28:588-95.

255 Roessler MS, Schmid DS, Michels P, et al. Early out-of-hospital non-invasive ventilation is superior to standard medical treatment in patients with acute respiratory failure: a pilot study. Emerg Med J 2012:29:409-14.

256 Templier F, Labastire L, Pes $\mathrm{P}$, et al. Noninvasive ventilation use in French out-of-hospital settings: a preliminary national survey. Am J Emerg Med 2012;30:765-9.

257 Rose L, Gerdtz MF. Non-invasive mechanical ventilation in Australian emergency departments: a prospective observational cohort study. Int I Nurs Stud 2009;46:617-23.

258 Hess DR, Pang JM, Camargo CA Jr. A survey of the use of noninvasive ventilation in academic emergency departments in the United States. Respir Care 2009:54:1306-12.

259 Browning J, Atwood B, Gray A, et al. Use of non-invasive ventilation in UK emergency departments. Emerg Med I 2006;23:920-1.

260 Sen B, Woollard M, Desira NL. Does the introduction of a COPD pro-forma improve the standards of care delivered by junior doctors in the emergency department. COPD 2010;7:199-203.

261 Sinuff T, Kahnamoui K, Cook DJ, et al. Practice guidelines as multipurpose tools: a qualitative study of noninvasive ventilation. Crit Care Med 2007:35:776-82.

262 NICE quality standard [QS10]. Chronic obstructive pulmonary disease in adults. 2011. https://www.nice.org.uk/guidance/qs10

263 Nava S, Hill N. Non-invasive ventilation in acute respiratory failure. Lancet 2009;374:250-9.

264 Corrado A, Roussos C, Ambrosino N, et al. Respiratory intermediate care units: a European survey. Eur Respir J 2002;20:1343-50.

265 Intensive Care Society. Levels of critical care for adult patients. London: Intensive Care Society, 2009.
266 BTS. The use of non-invasive ventilation in the management of patients with chronic obstructive pulmonary disease admitted to hospital with acute type II respiratory failure (with particular reference to bilevel positive pressure ventilation). London: BTS, 2008. https://www.brit-thoracic.org.uk/guidelines-and-qualitystandards/non-invasive-ventilation-(niv)/

267 Sala E, Balaguer C, Carrera M, et al. [Activity of an intermediate respiratory care unit attached to a respiratory medicine department]. Arch Bronconeumol 2009:45:168-72.

268 Paus-Jenssen ES, Reid JK, Cockcroft DW, et al. The use of noninvasive ventilation in acute respiratory failure at a tertiary care center. Chest 2004;126:165-72.

269 Royal College of Physicians National COPD Audit Programme. 2014. https://www. rcplondon.ac.uk/projects/national-copd-audit-programme

270 Cabrini L, Antonelli M, Savoia G, et al. Non-invasive ventilation outside of the Intensive Care Unit: an Italian survey. Minerva Anestesiol 2011;77:313-22.

271 Walters GG, S, Beauchamp B, et al. Seasonal infulenza: impact on NIV admissions and NIV failures. Thorax 2009;64:A30-31.

272 WHO definition of palliative care. http://www.who.int/cancer/palliative/definition/en

273 Momen N, Hadfield P, Kuhn I, et al. Discussing an uncertain future: end-of-life care conversations in chronic obstructive pulmonary disease. A systematic literature review and narrative synthesis. Thorax 2012;67:777-80.

274 Advanced Care Planning: A Guide for Health and Social Care Staff. 2007. http:// www.ncpc.org.uk/sites/default/files/AdvanceCarePlanning.pdf

275 Etheridge Z, Gatland E. When and how to discuss "do not resuscitate" decisions with patients. BMJ 2015;350:h2640

276 Chakrabarti B, Sulaiman MI, Davies L, et al. A study of patient attitudes in the United Kingdom toward ventilatory support in chronic obstructive pulmonary disease. J Palliat Med 2009;12:1029-35.

277 Sinuff T, Cook DJ, Keenan SP, et al. Noninvasive ventilation for acute respiratory failure near the end of life. Crit Care Med 2008;36:789-94.

278 Bulow HH, Thorsager B. Non-invasive ventilation in do-not-intubate patients: five-year follow-up on a two-year prospective, consecutive cohort study. Acta Anaesthesiol Scand 2009;53:1153-7.

279 Rocker GM, Dodek PM, Heyland DK, et al. Toward optimal end-of-life care for patients with advanced chronic obstructive pulmonary disease: insights from a multicentre study. Can Respir J 2008:15:249-54.

280 Pang SM, Chan KS, Chung BP, et al. Assessing quality of life of patients with advanced chronic obstructive pulmonary disease in the end of life. J Palliat Care 2005;21:180-7.

281 Smith TA, Davidson PM, Lam LT, et al. The use of non-invasive ventilation for the relief of dyspnoea in exacerbations of chronic obstructive pulmonary disease; a systematic review. Respirology 2012;17:300-7.

282 BMA. Withholding and withdrawing life-prolonging medical treatment. A guide for decision making. Oxford: Blackwell Publishing, 2007.

283 Simonds AK. Ethics and decision making in end stage lung disease. Thorax 2003;58:272-7.

284 Beck S, van de Loo A, Reiter-Theil S. A "little bit illegal"? Withholding and withdrawing of mechanical ventilation in the eyes of German intensive care physicians. Med Health Care Philos 2008;11:7-16.

285 Aita K, Kai I. Physicians' psychosocial barriers to different modes of withdrawal of life support in critical care: a qualitative study in Japan. Soc Sci Med 2010;70:616-22.

286 Lanken PN, Terry PB, Delisser HM, et al. An official American Thoracic Society clinical policy statement: palliative care for patients with respiratory diseases and critical illnesses. Am J Respir Crit Care Med 2008;177:912-27.

287 Lautrette A, Darmon M, Megarbane B, et al. A communication strategy and brochure for relatives of patients dying in the ICU. N Engl I Med 2007;356:469-78.

288 Cox C, Govert J, Shanawani H, et al. Providing palliative care for patients receiving mechanical ventilation in the intensive care unit. Part 2: withdrawing ventilation. Prog Palliat Care 2005;13:133-7.

289 Kuhnlein P, Kubler A, Raubold S, et al. Palliative care and circumstances of dying in German ALS patients using non-invasive ventilation. Amyotroph Lateral Scler 2008;9:91-8.

290 NICE interventional procedures guidance [IPG428]. Extracorporeal membrane carbon dioxide removal. http://www.nice.org.uk/guidance/ipg428.

291 Bein T, Weber-Carstens S, Goldmann A, et al. Lower tidal volume strategy (approximately $3 \mathrm{ml} / \mathrm{kg}$ ) combined with extracorporeal $\mathrm{CO} 2$ removal versus 'conventional' protective ventilation $(6 \mathrm{ml} / \mathrm{kg})$ in severe ARDS: the prospective randomized Xtravent-study. Intensive Care Med 2013;39:847-56.

292 Del Sorbo L, Pisani L, Filippini C, et al. Extracorporeal Co2 removal in hypercapnic patients at risk of noninvasive ventilation failure: a matched cohort study with historical control. Crit Care Med 2015:43:120-7.

293 Hurford WE, Cheifetz IM. Respiratory controversies in the critical care setting. Should heliox be used for mechanically ventilated patients? Respir Care 2007:52:582-91; discussion 91-4.

294 Manthous CA, Hall JB, Caputo MA, et al. Heliox improves pulsus paradoxus and peak expiratory flow in nonintubated patients with severe asthma. Am J Respir Crit Care Med 1995;151(2 Pt 1):310-14. 
295 Hess DR, Acosta FL, Ritz RH, et al. The effect of heliox on nebulizer function using a beta-agonist bronchodilator. Chest 1999;115:184-9.

296 Kumar D, Saksena RK. Best evidence topic report. Use of Heliox in the management of acute exacerbation of COPD. Emerg Med I 2007;24: 45-8.

297 Maggiore SM, Richard JC, Abroug F, et al. A multicenter, randomized trial of noninvasive ventilation with helium-oxygen mixture in exacerbations of chronic obstructive lung disease. Crit Care Med 2010;38:145-51.

298 Gluck EH, Onorato DJ, Castriotta R. Helium-oxygen mixtures in intubated patients with status asthmaticus and respiratory acidosis. Chest 1990;98: 693-8.

299 Colebourn CL, Barber V, Young JD. Use of helium-oxygen mixture in adult patients presenting with exacerbations of asthma and chronic obstructive pulmonary disease: a systematic review. Anaesthesia 2007;62: $34-42$.

300 Jaber S, Fodil R, Carlucci A, et al. Noninvasive ventilation with helium-oxygen in acute exacerbations of chronic obstructive pulmonary disease. Am J Respir Crit Care Med 2000;161(No.4):1191-200.

\section{APPENDIX 1 GUIDELINE GROUP MEMBERS}

Chair: Craig Davidson

Writing Groups

Acute NIV

Group lead: Mark Elliott,

Members: Colin Church, Tim Felton, Milind Sovani, Lynn McDonnell

Invasive Mechanical Ventilation

Group lead: Daniel Kennedy

Members: Ben Creagh-Brown, Craig Davidson, Alastair Glossop, Leigh Mansfield,

Care Planning

Group lead: Steve Banham

Members: Rob Parker, Craig Davidson, Ben Creagh-Brown, Lynn Thomas, Bernard Foëx 


\section{Correction}

Davidson AC, Banham S, Elliott M, et al. BTS/ICS guideline for the ventilatory management of acute hypercapnic respiratory failure in adults. Thorax 2016;71 Suppl 2:ii1-35. doi: 10.1136/thoraxjnl-2015-208209.

The British Thoracic Society wishes to clarify reference to the definition of hypercapnia in relation to the BTS/ICS Guidelines for the ventilatory management of acute hypercapnic respiratory failure (2016).

The British Thoracic Society recognises the definition of hypercapnia as a PaCO $2 \geq 6 \mathrm{kPa}$ as used in the BTS Standards of Care document on non invasive ventilation in acute respiratory failure $^{1}$ and BTS Guidelines for Emergency Oxygen Use in Adults. ${ }^{62}$

Previous guidelines recommended that NIV be considered if $\mathrm{pH}<7.35$ and $\mathrm{PaCO} 2>6 \mathrm{kPa}$ and $\mathrm{RR}>23$ breaths/min. These were predominantly written for patients with exacerbations of Chronic Obstructive Pulmonary Disease. ${ }^{48}$ NIV use in the UK has since broadened to treat a number of other diagnoses where the evidence for benefit is less robust and where sometimes there is a mixed metabolic and respiratory acidosis.

In the 2016 BTS/ICS Guidelines for the ventilatory management of acute hypercapnic respiratory failure, the guideline development group considered that in patients with type 2 respiratory failure, a PaCO2 between 6.0 and $6.5 \mathrm{kPa}$ is unlikely to make a large contribution to acidosis. Consensus within the guideline development group and open consultation on the draft guidelines suggested that these patients should receive optimal medical care and controlled flow oxygen while NIV is considered.

The convention and guidance in many centres has evolved to limit the widespread use of NIV in acidosis with a large metabolic contribution and to initiate NIV only in those patients where repeat arterial blood gas measurement confirms a persisting respiratory acidosis $\mathrm{pH}$ $<7.35$ with a higher threshold for $\mathrm{PaCO} 2>6.5 \mathrm{kPa}$.

The BTS/ICS Guidelines for the ventilatory management of acute hypercapnic respiratory failure recommendation 25 is amended to:

NIV should be started when a $\mathrm{pH}<7.35$, a PaCO2 of $\geq 6.5 \mathrm{kPa}$ and $\mathrm{RR}>23$ breaths/mins persists or develops after an hour of optimal medical therapy. (Grade A)

For patients with a PaCO2 between 6.0 and $6.5 \mathrm{kPa}$ NIV should be considered.(Grade D).

The following corrections are also noted:

Page 6 - Definition of AHRF:

"Conventionally a $\mathrm{pH}<7.35$ and a $\mathrm{PCO} 2>6.0 \mathrm{kPa}$ confirms acute respiratory acidosis and, when persisting after initial medical therapy, have been used as threshold values for considering the use of non-invasive ventilation."

Page 16:

"In around 20\% of AHRF cases secondary to AECOPD, optimised medical therapy, which includes targeting an oxygen saturation to $88-92 \%$, will result in normalisation of arterial $\mathrm{pH}^{2}{ }^{62}$ Established guidance is to await improvement and initiate NIV if, after $60 \mathrm{~min}$, the following are present: $\mathrm{pH}<7.35$, $\mathrm{pCO} 2>6.0 \mathrm{kPa}$ and $\mathrm{RR}>23$ breaths $/ \mathrm{min} .{ }^{1}{ }^{48}$

Thorax 2017;72:588. doi:10.1136/thoraxjnl-2015-208209corr1

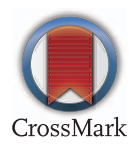

REFERENCES

1 British Thoracic Society Standards of Care Committee. Non-invasive ventilation in acute respiratory failure. Thorax 2002;57:192-211.

2 Plant PK, Owen J, Elliott MW. One year period prevalence study of respiratory acidosis in acute exacerbation of COPD; implications for the provision of non-invasive ventilation and oxygen administration. Thorax 2000;55:550-4.

48 NICE clinical guidance [CG12]. Chronic obstructive pulmonary disease - Management of chronic obstructive pulmonary disease in adults in primary and secondary care. 2004. http://www.nice.org.uk/guidance/cg12

62 O'Driscoll BR, Howard LS, Davison AG, et al. BTS guideline for emergency oxygen use in adult patients. Thorax 2008;63: (Suppl_6):vi1-68. 